\title{
Second-Moment RANS Model Verification and Validation using the Turbulence Modeling Resource Website (Invited)
}

\author{
Bernhard Eisfeld \\ ${ }^{*}$ DLR Institute of Aerodynamics and Flow Technology, Braunschweig, D-38108, Germany \\ Chris Rumsey \\ ${ }^{\dagger}$ NASA Langley Research Center, MS 128, Hampton, VA, USA \\ Vamshi Togiti \\ ${ }^{\ddagger}$ DLR Institute of Aerodynamics and Flow Technology, Braunschweig, D-38108, Germany
}

The implementation of the SSG/LRR- $\omega$ differential Reynolds stress model into the NASA flow solvers CFL3D and FUN3D and the DLR flow solver TAU is verified by studying the grid convergence of the solution of three different test cases from the Turbulence Modeling Resource Website. The model's predictive capabilities are assessed based on four basic and four extended validation cases also provided on this website, involving attached and separated boundary layer flows, effects of streamline curvature and secondary flow. Simulation results are compared against experimental data and predictions by the eddyviscosity models of Spalart-Allmaras (SA) and Menter's Shear Stress Transport (SST).

\section{Nomenclature}

$\begin{array}{ll}a & \text { Sound speed, } \mathrm{m} / \mathrm{s} \\ b_{i j} & \text { Cartesian component of the anisotropy tensor } \\ C & \text { Intercept of log-law } \\ C_{i} & \text { Coefficients of pressure-strain correlation } \\ C_{i}^{*} & \text { Coefficients of pressure-strain correlation } \\ C_{D} & \text { Drag coefficient } \\ C_{L} & \text { Lift coefficient } \\ C_{p} & \text { Pressure coefficient } \\ \Delta C_{p} & \text { Shift in pressure coefficient } \\ C_{\mu} & \text { Coefficient for } \epsilon / \omega \text { conversion } \\ c & \text { Chord length, } \mathrm{m} \\ c_{2}^{(L R R)} & \text { Coefficient of pressure-strain correlation (LRR-model) } \\ c_{f} & \text { Local skin friction coefficient } \\ D & \text { Height of curved duct, } \mathrm{m} \\ D_{i j} & \text { Cartesian component of diffusion tensor, } \mathrm{m}^{2} / \mathrm{s}^{3} \\ D^{(G D)} & \text { Coefficient of generalized gradient diffusion model } \\ D^{(S D)} & \text { Coefficient of simple gradient diffusion model } \\ d & \text { Distance to closest wall, } \mathrm{m} \\ F_{1} & \text { Menter's blending function }\end{array}$

\footnotetext{
${ }^{*}$ Research Scientist, Dept. $\mathrm{C}^{2} \mathrm{~A}^{2} \mathrm{~S}^{2} \mathrm{E}$, Member AIAA.

${ }^{\dagger}$ Research Scientist, Computational AeroSciences Branch, Fellow AIAA.

$\ddagger$ Research Scientist, Dept. $\mathrm{C}^{2} \mathrm{~A}^{2} \mathrm{~S}^{2} \mathrm{E}$.
} 


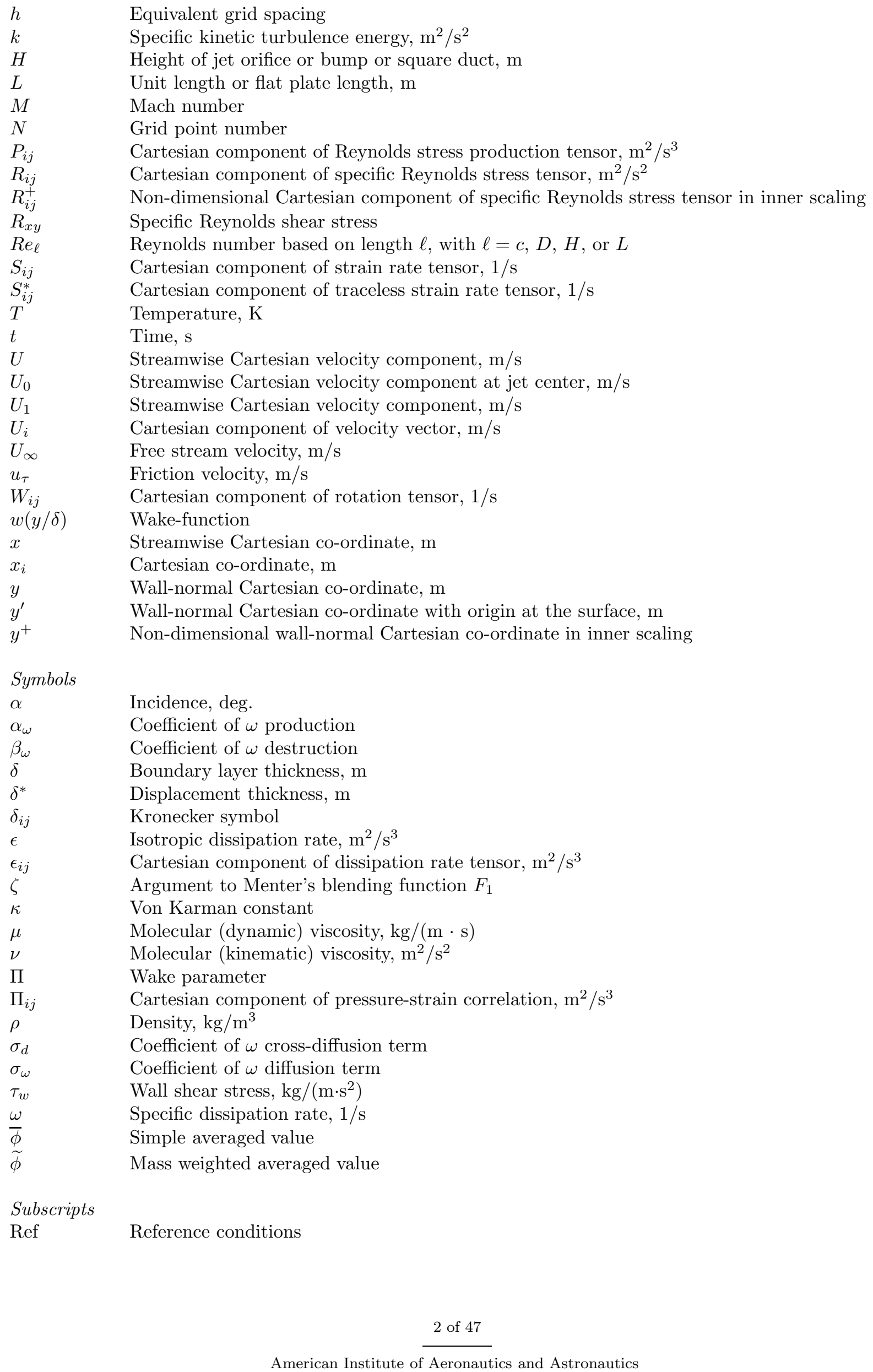




\section{Introduction}

Computational Fluid Dynamics (CFD) is routinely applied in contemporary aircraft design. Nevertheless, the predicted result of any flow simulation depends on the accuracy of the underlying representation of the flow physics, i.e., the effect of turbulence, and the accuracy of the numerical method solving the corresponding equations.

While the numerical error, at least in principle, can be reduced by refining the computational mesh and, in time-accurate simulations, the time step, reducing the modeling error is much more demanding. Theoretically, a turbulence model can be completely avoided by performing a Direction Numerical Simulation (DNS), which then requires resolving all turbulent fluctuations down to the smallest scales. Alternatively, in a Large Eddy Simulation (LES), the resolution requirements can be relaxed, but at the expense of modeling the effect of the smallest scales. Unfortunately, the size of the smallest scales to be resolved decreases rapidly with increasing Reynolds number, currently restricting the application of such scale resolving methods. ${ }^{1}$

For this reason, turbulence models based on the Reynolds-averaged Navier-Stokes (RANS) equations are still the backbone of industrially applied CFD methods (for an overview see Ref. 2). Within this framework, only the mean flow is considered, where the net effect of turbulence enters into the momentum balance via additional Reynolds stresses. As a consequence, these additional unknowns need to be expressed in terms of known mean quantities, which constitutes the art of RANS turbulence modeling.

The most widespread RANS modeling approach is based on the Boussinesq hypothesis, assuming the turbulence to effectively increase the viscosity of the fluid by a flow dependent eddy viscosity. ${ }^{2}$ Thus, only one single parameter needs to be provided that can be calibrated to correctly predict (for example) the turbulent shear stress in a boundary layer. Indeed, eddy-viscosity models (EVM) like the Spalart-Allmaras (SA) model $^{3}$ or the Menter Shear Stress Transport (SST) model ${ }^{4}$ have proven to give reliable predictions in a wide range of applications and are therefore heavily used within the aeronautics industry.

However, pushing the applications to the borders of flight, the accuracy of the applied eddy-viscosity models degrades, where the prediction of flow separation appears to be the greatest challenge. One possibility for improvement consists in replacing the Boussinesq hypothesis by solving the individual transport equations for the Reynolds stresses. Corresponding Second-Moment Closures (SMC), also known as Reynolds stress models (RSM), allow for a more detailed representation of the flow physics, but nevertheless require modeling of individual terms in the underlying transport equations (for an overview see Ref. 5). Hence, as with eddyviscosity models, the accuracy of Reynolds stress models needs to be assessed individually.

The SSG/LRR- $\omega$ Reynolds stress model $^{6}$ has been demonstrated to be applicable to fairly complex aeronautical flow problems. It has therefore been selected by NASA and DLR for a common verification and validation exercise based on the test cases on the Turbulence Modeling Resource (TMR) Website. ${ }^{7}$

\section{SSG/LRR- $\omega$ Model}

The SSG/LRR- $\omega$ model has been developed within the EU-project FLOMANIA ${ }^{8}$ and, as any Reynolds stress model, is based on the Reynolds stress transport equation

$$
\frac{\partial\left(\bar{\rho} \widetilde{R}_{i j}\right)}{\partial t}+\frac{\partial}{\partial x_{k}}\left(\bar{\rho} \widetilde{R}_{i j} \widetilde{U}_{k}\right)=\bar{\rho} P_{i j}+\bar{\rho} \Pi_{i j}-\bar{\rho} \epsilon_{i j}+\bar{\rho} D_{i j},
$$

where $\bar{\rho}$ is the mean density, $\widetilde{U}_{i}$ represents the Cartesian components of the mean velocity vector and $\bar{\rho} \widetilde{R}_{i j}$ the Cartesian components of the Reynolds stress tensor.

The Cartesian components of the production term are given by

$$
\bar{\rho} P_{i j}=-\bar{\rho} \widetilde{R}_{i k} \frac{\partial \widetilde{U}_{j}}{\partial x_{k}}-\bar{\rho} \widetilde{R}_{j k} \frac{\partial \widetilde{U}_{i}}{\partial x_{k}}
$$

and do not need modeling, since all quantities are provided by the system of equations to be solved.

The Cartesian components of the pressure-strain correlation are formally modeled according to Speziale, Sarkar and Gatski (SSG) ${ }^{9}$ as

$$
\bar{\rho} \Pi_{i j}=-\left(C_{1} \bar{\rho} \epsilon+\frac{1}{2} C_{1}^{*} \bar{\rho} P_{k k}\right) \widetilde{b}_{i j}+C_{2} \bar{\rho} \epsilon\left(\widetilde{b}_{i k} \widetilde{b}_{k j}-\frac{1}{3} \widetilde{b}_{k l} \widetilde{b}_{k l} \delta_{i j}\right)+\left(C_{3}-C_{3}^{*} \sqrt{\widetilde{b}_{k l} \widetilde{b}_{k l}}\right) \bar{\rho} \bar{k} \widetilde{S}_{i j}^{*}
$$




$$
+C_{4} \bar{\rho} \widetilde{k}\left(\widetilde{b}_{i k} \widetilde{S}_{j k}+\widetilde{b}_{j k} \widetilde{S}_{i k}-\frac{2}{3} \widetilde{b}_{k l} \widetilde{S}_{k l} \delta_{i j}\right)+C_{5} \bar{\rho} \widetilde{k}\left(\widetilde{b}_{i k} \widetilde{W}_{j k}+\widetilde{b}_{j k} \widetilde{W}_{i k}\right)
$$

where $\widetilde{k}=\widetilde{R}_{i i} / 2$ is the specific kinetic turbulence energy and

$$
\widetilde{b}_{i j}=\frac{\widetilde{R}_{i j}}{2 \widetilde{k}}-\frac{1}{3} \delta_{i j}
$$

represents the Cartesian components of the anisotropy tensor. Furthermore

$$
\begin{aligned}
\widetilde{S}_{i j} & =\frac{1}{2}\left(\frac{\partial \widetilde{U}_{i}}{\partial x_{j}}+\frac{\partial \widetilde{U}_{j}}{\partial x_{i}}\right), \\
\widetilde{S}_{i j}^{*} & =\widetilde{S}_{i j}-\frac{1}{3} \widetilde{S}_{k k} \delta_{i j}, \\
\widetilde{W}_{i j} & =\frac{1}{2}\left(\frac{\partial \widetilde{U}_{i}}{\partial x_{j}}-\frac{\partial \widetilde{U}_{j}}{\partial x_{i}}\right)
\end{aligned}
$$

denote the Cartesian components of the simple strain rate tensor, the traceless strain rate tensor and the rotation tensor, respectively. The $C_{i}$ and $C_{i}^{*}$ are model dependent coefficients.

Dissipation is modeled as an isotropic tensor with Cartesian components

$$
\bar{\rho} \epsilon_{i j}=\frac{2}{3} \bar{\rho} \epsilon,
$$

where the isotropic dissipation rate $\epsilon$ is provided indirectly by a transport equation for the specific dissipation rate $\omega$ given further below.

Two different models are available representing non-convective fluxes, simple gradient diffusion ${ }^{10}$

$$
\bar{\rho} D_{i j}=\frac{\partial}{\partial x_{k}}\left[\left(\bar{\mu}+\frac{D^{(S D)}}{C_{\mu}} \frac{\bar{\rho} \widetilde{k}^{2}}{\epsilon}\right) \frac{\partial \widetilde{R}_{i j}}{\partial x_{k}}\right]
$$

or generalized gradient diffusion ${ }^{11}$

$$
\bar{\rho} D_{i j}=\frac{\partial}{\partial x_{k}}\left[\left(\bar{\mu} \delta_{i j}+D^{(G D)} \frac{\bar{\rho} \widetilde{k} \widetilde{R}_{k l}}{\epsilon}\right) \frac{\partial \widetilde{R}_{i j}}{\partial x_{l}}\right],
$$

where $\bar{\mu}$ is the mean dynamic fluid viscosity and $D^{(S D)}$ and $D^{(G D)}$ represent respective model coefficients.

Finally, Menter's baseline equation ${ }^{4}$

$$
\frac{\partial(\bar{\rho} \omega)}{\partial t}+\frac{\partial}{\partial x_{k}}\left(\bar{\rho} \omega \widetilde{U}_{k}\right)=\alpha_{\omega} \frac{\omega}{\widetilde{k}} \frac{\bar{\rho} P_{k k}}{2}-\beta_{\omega} \bar{\rho} \omega^{2}+\frac{\partial}{\partial x_{k}}\left[\left(\bar{\mu}+\sigma_{\omega} \frac{\bar{\rho} \widetilde{k}}{\omega}\right) \frac{\partial \omega}{\partial x_{k}}\right]+\sigma_{d} \frac{\bar{\rho}}{\omega} \max \left(\frac{\partial \widetilde{k}}{\partial x_{k}} \frac{\partial \omega}{\partial x_{k}}, 0\right)
$$

is employed for providing the isotropic dissipation rate

$$
\epsilon=C_{\mu} \widetilde{k} \omega,
$$

where $C_{\mu}=0.09$. As with the SST model, the coefficients $\phi=\alpha_{\omega}, \beta_{\omega}, \sigma_{\omega}, \sigma_{d}$ are blended according to

$$
\phi=F_{1} \phi^{(\omega)}+\left(1-F_{1}\right) \phi^{(\epsilon)}
$$

between the bounding values associated with the $\omega$-equation by Wilcox ${ }^{12}$ near walls $\left(F_{1}=1\right)$ and the standard $\epsilon$-equation ${ }^{13}$ at the boundary layer edge $\left(F_{1}=0\right)$, where the blending function is given by ${ }^{4}$

$$
F_{1}=\tanh \left(\zeta^{4}\right)
$$

with the argument

$$
\zeta=\min \left[\max \left(\frac{\sqrt{\tilde{k}}}{C_{\mu} \omega d}, \frac{500 \bar{\mu}}{\bar{\rho} \omega d^{2}}\right), \frac{4 \sigma_{\omega}^{(\varepsilon)} \bar{\rho} \widetilde{k}}{\sigma_{d}^{(\varepsilon)} \frac{\bar{\rho}}{\omega} \max \left(\frac{\partial \widetilde{k}}{\partial x_{k}} \frac{\partial \omega}{\partial x_{k}}, 0\right) d^{2}}\right]
$$


Table 1. Bounding Values of $\omega$-Equation Coefficients

\begin{tabular}{ccccc} 
& $\alpha_{\omega}$ & $\beta_{\omega}$ & $\sigma_{\omega}$ & $\sigma_{d}$ \\
\hline$\phi^{(\varepsilon)}$ & 0.44 & 0.0828 & 0.856 & 1.712 \\
$\phi^{(\omega)}$ & 0.5556 & 0.075 & 0.5 & 0
\end{tabular}

based on the wall-distance $d$. The corresponding values of the coefficients are given in Table 1 .

The fundamental idea of the SSG/LRR- $\omega$ model is to combine the $\epsilon$-based SSG model for the pressurestrain correlation with the simpler model by Launder, Reece and Rodi (LRR) ${ }^{14}$ near walls in the $\omega$-based formulation of Wilcox. ${ }^{15}$ Since the LRR-model can be written as a subset of the above SSG-model, Eq. (3), the same blending of the Reynolds stress model coefficients $C_{i}, C_{i}^{*}, D^{(S D)}$ and $D^{(G D)}$ can be applied as to the coefficients of the $\omega$-equation. Thus, all coefficients change consistently from LRR $+\omega$ near walls to SSG+ $\epsilon$ at the boundary layer edge. The corresponding bounding values are given in Table 2 . Note that the value of the coefficient $c_{2}^{(L R R)}$ as well as the generalized diffusion coefficient $D^{(G D)}$ have been re-calibrated for better agreement with the log-law velocity profile in a zero pressure gradient boundary layer. ${ }^{16}$ Therefore, on the Turbulence Modeling Resource Website, ${ }^{7}$ the model is denoted SSG/LRR-RSM-w2012 when employing the generalized diffusion model and SSG/LRR-RSM-w2012-SD when employing the simple gradient diffusion model. In this paper only the generalized gradient diffusion model is used so that for brevity the model is termed SSG/LRR- $\omega$ only.

Table 2. Bounding Values of the SSG/LRR- $\omega$ Model Term Coefficients $\left(c_{2}^{(L R R)}=0.52\right)$

\begin{tabular}{cccccccccc} 
& $C_{1}$ & $C_{1}^{*}$ & $C_{2}$ & $C_{3}$ & $C_{3}^{*}$ & $C_{4}$ & $C_{5}$ & $D^{(S D)}$ & $D^{(G D)}$ \\
\hline SSG & 3.4 & 1.8 & 4.2 & 0.8 & 1.3 & 1.25 & 0.4 & $\frac{2}{3} 0.22$ & 0.22 \\
LRR & 3.6 & 0 & 0 & 0.8 & 0 & $\frac{18 c_{2}^{(L R R)}+12}{11}$ & $\frac{-14 c_{2}^{(L R R)}+20}{11}$ & $0.5 C_{\mu}$ & $0.75 C_{\mu}$
\end{tabular}

\section{Verification of Implementation}

\section{III.A. Method}

In this article, verification refers to the objective that a turbulence model, here the SSG/LRR- $\omega$, is implemented correctly, i.e., according to the specified model equations, including boundary conditions. ${ }^{7}$ Since giving a strict proof might be rather difficult, a more heuristic weak form of verification is applied, comparing simulation results obtained with independently developed flow solvers on a sequence of successively refined grids. Good agreement between different solutions in the limit of grid independence enhances the confidence in the correct implementation of the model into all of the tested codes. As already said, the comparison will be restricted to the 2012 version of the SSG/LRR- $\omega$ model with generalized gradient diffusion.

\section{III.B. Flow Solvers}

The SSG/LRR- $\omega$ model has been implemented independently into three different production codes of NASA and DLR: CFL3D, FUN3D and TAU, solving the compressible Reynolds-averaged Navier-Stokes equations together with the respective turbulence equations. CFL3D is a cell-centered structured-grid code developed at NASA, whereas FUN3D and TAU are node-centered unstructured-grid codes developed by NASA and DLR, respectively. All three are based on a finite-volume discretization of formally second order accuracy in space, where the preferred specific discretization scheme varies between the different codes and organizations. The advection terms of the turbulence equations are usually solved with first order accuracy only. Most cases to be shown below used this option. However, for a few of the cases, TAU also employed second order accuracy for the turbulence advection terms to explore their influence. 


\section{III.C. Results}

Three two-dimensional verification cases have been selected from the TMR Website: ${ }^{7}$ the flow over a flat plate at zero pressure gradient, the plane jet, and the flow over a bump. These cases are described in detail in the following sections.

\section{III.C.1. Flat plate at zero pressure gradient}

The flow over a flat plate is computed with the mean flow boundary conditions defined according to the TMR Website, Fig. 1. ${ }^{7}$ The Mach number is $M=0.2$, Reynolds number is $R e_{L}=10 \cdot 10^{6}$ based on the flat plate length $L$, and reference temperature is $T_{\text {Ref }}=300 \mathrm{~K}$. The computations have been carried out on the sequence of five structured grids, also provided on the TMR Website, ${ }^{7}$ ranging from $N=35 \times 25$ points on the coarsest level to $N=545 \times 385$ points on the finest level, where the coarser levels have been generated by omitting every second grid line in each coordinate direction. The TAU computations have been performed with two different spatial discretization schemes for the advection terms of the turbulence equations, allowing us to study the influence of this term on the overall numerical accuracy.

Figure 2 (left) shows the variation of the predicted total drag coefficient $C_{D}$ with grid resolution in terms of an equivalent grid spacing $h=1 / \sqrt{N}$. The differences between the predicted results reduce significantly, when refining the mesh, indicating one common solution in the limit of infinitely small cells. This is further verified by Fig. 2 (right), depicting the dependence of the local skin friction coefficient, $c_{f}$, at $x / L=0.485$ with the grid resolution. The differences between the different predictions on the finest mesh appear even smaller than for the total drag coefficient. Note that in agreement with boundary layer theory ${ }^{17}$ in this particular case, no advantage of second order accuracy for the advection terms can be observed on the finer grid levels.

As it turns out, the somewhat larger differences in total drag are due to differences in the vicinity of the flat plate leading edge only. This is revealed by Fig. 3, showing a log-log plot of the local skin friction coefficient over the flat plate predicted on the finest mesh. Larger differences are only observed up to $x / L \approx 5 \cdot 10^{-3}$, which can be attributed to the singularity at the leading edge.

The very good agreement between the different predictions on the finest mesh is confirmed, when comparing profiles of various quantities normal to the wall. Figure 4 shows the predicted velocity profiles at $x / L=0.485$ normal to the wall in inner scaling. The inner scaling is defined by relating all quantities to the kinematic viscosity, $\nu$, of the fluid and the friction velocity

$$
u_{\tau}=\sqrt{\frac{\tau_{w}}{\bar{\rho}}},
$$

where $\tau_{w}$ is the local shear stress at the wall and $\bar{\rho}$ is the fluid density. Corresponding quantities are indicated with a superscript ${ }^{+}$, i.e., $y^{+}=y u_{\tau} / \nu, u^{+}=U_{1} / u_{\tau}, R_{i j}^{+}=R_{i j} / u_{\tau}^{2}$, where the tilde symbol over $U_{1}$ and $R_{i j}$ has been dropped for convenience. Virtually no difference can be observed between the predicted velocity profiles over the complete boundary layer height.

Figure 5 shows the corresponding profiles of the non-dimensional Reynolds stresses in inner scaling at the same position, $x / L=0.485$. Small deviations can be observed only towards the boundary layer edge; in particular, the second-order turbulence advection terms steepen the gradients in this region. However, the agreement between the CFL3D and TAU results (both with first-order turbulence advection) is nearly perfect.

\section{III.C.2. Plane jet}

In this case, a plane jet is developing behind an orifice, from where fluid is entering into an ambient stream at lower uniform velocity. Corresponding computations have been carried out on the series of five structured meshes provided on the TMR Website ${ }^{7}$ using the boundary conditions as depicted in Fig. 6. Reference Mach number is $M_{R e f}=0.5$, Reynolds number is $R e_{H}=50,000$ based on height of the orifice, and reference temperature is $T_{R e f}=300 \mathrm{~K}$. Note that the orifice is modeled as an infinitely thin plate subjected to the flow on both sides.

The original grids are composed of three blocks with a total point number of $N=1,280$ on the coarsest up to $N=327,680$ on the finest level. The coarser grids are obtained from the respective next finer one by omitting every second grid line in both directions. 
Figure 7 (left) shows the variation of the total drag coefficient $C_{D}$ on the thin plate with the grid resolution in terms of an equivalent grid spacing $h=1 / \sqrt{N}$. As to be expected, the differences between the predicted values decreases with increasing mesh resolution. Nevertheless, even on the finest mesh there remains a very small difference.

Additionally, Fig. 7 (right) presents the non-dimensional streamwise velocity, $U_{0} / a_{R e f}$ (where $a_{R e f}$ is the reference speed of sound), on the centerline of the jet versus the equivalent grid spacing $h$ at three different positions $x / H$ downstream of the orifice. Once again, the differences between the predictions reduce with increasing mesh resolution, but the variation differs between the three positions. Interestingly, the largest differences remain at $x / H=29.2468$, which is located between the two other locations.

That the remaining differences are indeed very small can be seen from Fig. 8 showing the development of the non-dimensional streamwise velocity on the jet centerline along the flow computed on the finest mesh. On this scale, there is virtually no difference between the predictions by the different codes.

This observation is confirmed when comparing profiles of the non-dimensional velocity and Reynolds stresses across the jet. Figure 9 shows the corresponding results for the non-dimensional velocity profile on the finest mesh at position $x / H=29.2468$, where the largest differences have been observed in the centerline velocity. All results virtually collapse on a single line. A close-up of the velocity near the centerline reveals small differences that may reflect differences in the numerical treatment of symmetry planes in the different flow solvers. Similarly the non-dimensional Reynolds stress profiles, shown in Fig. 10, nearly agree perfectly.

\section{III.C.3. Two-dimensional bump in a channel}

The two-dimensional flow over a bump in a channel is a verification test case on the TMR Website ${ }^{7}$ aimed at an increased complexity of the flow field due to the variation of the pressure gradient over the bump. The contour of the lower channel wall is defined by ${ }^{7}$

$$
\frac{y}{H}=\left\{\begin{array}{ll}
0 & x / H \leq 6 \\
\left\{\sin \left[\frac{\pi}{3}\left(\frac{1}{6} \frac{x}{H}-1\right)\right]\right\}^{4} & 6 \leq x / H \leq 24 \\
0 & x / H \geq 24
\end{array},\right.
$$

where $H$ is the height of the bump. It is shown in Fig. 11 together with the corresponding boundary conditions. The Mach number is $M=0.2$, Reynolds number $R_{H}=150,000$ based on the bump height, and reference temperature is $T_{R e f}=300 \mathrm{~K}$.

Computations have been carried out on the series of five structured meshes provided on the TMR Website ${ }^{7}$ with point numbers ranging from $N=89 \times 41$ on the coarsest to $N=1,409 \times 641$ on the finest level. As usual, the coarser grids have been obtained by omitting every second grid line of the respective next finer level.

Figure 12 shows the variation of different total force coefficients with the equivalent grid spacing $h=$ $1 / \sqrt{N}$. In particular, the differences in the lift coefficient $C_{L}$, Fig. 12 (left), and the pressure drag coefficient $C_{D, p}$, Fig. 12 (right), are decreasing with increased mesh resolution. For the viscous drag coefficient $C_{D, v}$, Fig. 12 (right), the effect is less prominent, in particular when plotted to the same scales as the pressure drag coefficient. Nevertheless, Fig. 13 shows that the local skin friction coefficient at three positions around the crest of the bump converges to almost identical values with all three codes.

Figure 14 shows the distributions of the local pressure coefficient $C_{p}$ (left) and the local skin friction coefficient $c_{f}$ (right) along the viscous wall, computed on the finest mesh with $N=1,409 \times 641$ points. The results are virtually identical, except for some differences in $c_{f}$ near the inflow plane that vanish rapidly.

Figure 15 shows the profile of the streamwise velocity in inner scaling at $x / H=24$, corresponding to the end of the hump. At this position the complete history effects of the flow over the bump are expected to be present. Compared to the flow over a flat plate at zero pressure gradient, Fig. 4, the velocity profile is largely distorted, and the corresponding Reynolds stresses in Fig. 16 show a significant peak close to the boundary layer edge. Nevertheless, all predictions from the three codes virtually collapse to a single result except for some insignificant differences near the Reynolds stress extrema.

\section{III.D. Summary of Verification}

In all three considered verification cases, flat plate, plane jet and bump flow, the predictions of CFL3D, FUN3D and TAU apparently converge towards the same solution. While integral indicators, like total force 
coefficients, still show some very small remaining differences on the respective finest mesh, there is hardly any difference visible when plotting skin friction or pressure distributions in standard scales. Furthermore, very good agreement has been observed in velocity and Reynolds stress profiles.

Therefore, there is high confidence that the SSG/LRR- $\omega$ model has been implemented into all three codes correctly according to its specification. Under the assumption of a sufficiently fine grid and identical boundary conditions, predictions with any of these codes should yield the same results for this turbulence model. On arbitrary grids, however, differences in the results may be observed due to differences in the numerical methods. For this reason, results for the following validation cases will be cross-checked as far as possible.

\section{Validation}

In this article, validation refers to the question of how well real flows are represented by a turbulence model, e.g., the SSG/LRR- $\omega$ model. Therefore, in this section, the focus is on comparisons of numerical simulation results with experimental data. According to the TMR Website, ${ }^{7}$ the different test cases will be separated into basic and extended ones, where the focus will be on wall-bounded flows. Based on the experience presented on the TMR Website, computations on the second finest grid level are usually considered sufficient. SSG/LRR- $\omega$ model predictions with TAU, CFL3D, and/or FUN3D will be presented to get an indication of any possible remaining numerical influence. Additionally, results obtained with CFL3D using the well-established Spalart-Allmaras (SA) model ${ }^{3}$ and the Shear Stress Transport (SST) model by Menter ${ }^{4}$ are included for comparison. The verification of these models in CFL3D is documented on the TMR Website.

\section{IV.A. Basic Validation Cases}

\section{IV.A.1. Flat plate at zero pressure gradient}

The flow over a flat plate at zero pressure gradient has also been selected as a basic validation case, where the fine grid results of the above verification study are used. The flow conditions refer to a Reynolds number of $R e_{L}=10 \cdot 10^{6}$ based on the total length $L$ of the flat plate, corresponding to the experimental conditions of Wieghardt. ${ }^{18}$ According to the TMR Website, the Mach number is set to $M=0.2$ and reference temperature is $300 \mathrm{~K} .{ }^{7}$ Numerical results for the SSG/LRR- $\omega$ model on the fine grid are taken from the above verification study and are complemented by fine grid results obtained with CFL3D, using the SA and the SST models.

Figure 17 (left) shows the predicted velocity profiles in inner scaling, extracted at $x / L=0.95$, compared to the analytical expressions for the viscous sublayer

$$
u^{+}=y^{+}
$$

valid up to $y^{+} \approx 5$, and the so-called log-law

$$
u^{+}=\frac{1}{\kappa} \ln y^{+}+C
$$

with standard values for the von Kármán constant $\kappa=0.41$ and the intercept $C=5$. The importance of the log-law lies in the fact that it is apparently valid in any wall-bounded incompressible turbulent flow, independent of the Reynolds number. ${ }^{19}$ There is close agreement between the SST and the SSG/LRR$\omega$ predictions over almost the whole boundary layer height. The SA model predicts a smaller buffer layer between the viscous sublayer and the log-law region, which is probably in better agreement with experimental observation, although not shown here. Closer inspection of the region $100 \leq y^{+} \leq 1000$, depicted in Fig. 17 (right), reveals that all models predict a slightly steeper velocity gradient than would follow from the log-law with $\kappa=0.41$.

Figure 18 shows the skin friction distribution along the flat plate predicted by the different turbulence models compared to the experimental results by Wieghardt ${ }^{18}$ as provided by Coles and Hirst. ${ }^{20}$ All predictions are close to one another and, at least for lower local Reynolds number, tend to slightly underestimate the Wieghardt data. Closer inspection of the high Reynolds number region, Fig. 18 (right), reveals that the SSG/LRR- $\omega$ result virtually coincides with the SST result, whereas the SA model gives slightly higher skin friction, which apparently is in better agreement with the Wieghardt data. Nevertheless, the differences seem to be small, and all three turbulence model results appear acceptable. 
Note that the skin friction is an indicator for the boundary layer thickness, $\delta$, and correspondingly the displacement thickness, $\delta^{*}$, as can be seen from the analytical velocity profile suggested by Coles $^{21}$

$$
u^{+}=\frac{1}{\kappa} \ln y^{+}+C+\frac{\Pi}{\kappa} w(y / \delta),
$$

where $w(y / \delta)$ is the wake function with the limiting values

$$
\begin{aligned}
& w(y / \delta=0)=0 \\
& w(y / \delta=1)=2
\end{aligned}
$$

and the integral

$$
\int_{0}^{1} w d(y / \delta)=1
$$

The wake function is supposed to be universal, but is scaled with the flow dependent wake parameter $\Pi$, assuming a value of $\Pi=0.55$ for the boundary layer of a flat plate. ${ }^{21}$ The Coles profile, Eq. (20), describes the velocity profile in the whole boundary layer, except in the viscous sublayer and the buffer layer near walls, i.e., below $y^{+} \approx 30-50$.

Evaluating the velocity profile, Eq. (20), at the boundary layer edge, $y=\delta$, and noting that $u_{\infty}^{+}=$ $U_{\infty} / u_{\tau}=\sqrt{2 / c_{f}}$ (where $\infty$ represents the farfield reference conditions), one can re-write Eq. (20) to obtain an equation for the boundary layer thickness non-dimensionalized with the flat plate length $L$

$$
\frac{\delta}{L}=\frac{1}{R e_{L}} \sqrt{\frac{2}{c_{f}}} \exp \left\{\kappa \sqrt{\frac{2}{c_{f}}}-(\kappa C+2 \Pi)\right\},
$$

where $R e_{L}$ is the Reynolds number based on the flat plate length. Clearly, the larger the skin friction coefficient, $c_{f}$, the smaller the boundary layer thickness, $\delta$. As shown by Coles,$^{20}$ the displacement thickness, $\delta^{*}$, can be related to the boundary layer thickness, $\delta$, by

$$
\frac{\delta^{*} U_{\infty}}{\delta u_{\tau}}=\int_{0}^{1} \frac{U_{\infty}-U}{u_{\tau}} d(y / \delta)=\frac{1+\Pi}{\kappa}
$$

ignoring the deviation of the velocity profile, Eq. (20), from the viscous sublayer relation near the wall. After some re-arrangement one obtains

$$
\frac{\delta^{*}}{L}=\frac{1}{R e_{L}} \frac{1+\Pi}{\kappa} \exp \left\{\kappa \sqrt{\frac{2}{c_{f}}}-(\kappa C+2 \Pi)\right\},
$$

showing that the displacement thickness, $\delta^{*}$, also decreases with increasing skin friction coefficient, $c_{f}$.

Figure 19 shows the non-dimensional boundary layer thickness, $\delta / L$, (left) and the non-dimensional displacement thickness, $\delta^{*} / L$, (right) computed from the theoretical equations, Eqs. (24) and (26), respectively, for $R e_{L}=10 \cdot 10^{6}$, using the local skin friction coefficient $c_{f}$ of the above numerical predictions. As might have been deduced, the SA model yields slightly lower values of $\delta / L$ and $\delta^{*} / L$ than the SST and the SSG/LRR- $\omega$ model, which is supposed to have an effect on the prediction of shock positions in transonic flow; see Ref. 16. The larger $c_{f}$, the smaller the displacement thickness, $\delta^{*}$, and the further downstream a shock is expected to be predicted.

\section{IV.A.2. Subsonic Flow Around the NACA 0012 Airfoil}

The subsonic flow around the NACA 0012 airfoil is another basic validation test case documented on the TMR Website. ${ }^{7}$ It is considered representative of mostly attached flows around airfoils and slender bodies. According to the TMR Website, ${ }^{7}$ the inflow conditions are defined by a Mach number of $M=0.15$ and a Reynolds number of $R e_{c}=6 \cdot 10^{6}$ based on the chord length, $c$, of the airfoil. The reference temperature is taken as $T_{R e f}=300 \mathrm{~K}$.

Numerical predictions have been carried out, using CFL3D and TAU on the second finest structured mesh with $N=897 \times 257$ points provided on the TMR Website. ${ }^{7}$ The boundary conditions employed are sketched in Fig. 20, also taken from the TMR Website. ${ }^{7}$ Note that the farfield is approximately 500 chord 
lengths away from the airfoil, in order to minimize the effect of the lift generating surface on the farfield boundary condition inflow.

According to the TMR Website, the numerical results are provided at three different incidences only, i.e., $\alpha=0^{\circ}, \alpha=10^{\circ}$, and $\alpha=15^{\circ}$. Some complementary results have been computed with TAU at $\alpha=1^{\circ}$, $\alpha=2^{\circ}$, and $\alpha=5^{\circ}$, using the SSG/LRR- $\omega$ model.

Figure 21 (left) shows the lift coefficient, $C_{L}$, versus the incidence as computed with CFL3D and TAU, using different turbulence models, compared to the experimental data by Ladson ${ }^{22}$ provided on the TMR Website. ${ }^{7}$ Note that all computations have been carried out in fully turbulent mode, whereas in the experiment transition was tripped. Nevertheless, according to the TMR Website, ${ }^{7}$ these data appear to be the most suitable for comparison of force coefficients. ${ }^{7}$

Obviously, there is good agreement between all different predictions and the experiment for the lift curve, Fig. 21 (left). A detailed view of the results at higher incidence in Fig. 21 (right) reveals that with the SA model at $\alpha=15^{\circ}$, the lift is predicted slightly higher than with the SST and the SSG/LRR- $\omega$ model, which seem to be a little closer to the experimental lift curve. This difference is also observed in the force polar, depicted in Fig. 22, where the SA prediction might be considered in slightly better agreement with the measurement. However, the numerical results are too sparse for a more definite conclusion.

The reason for the small differences between turbulence models becomes clear when looking at the pressure distributions at $\alpha=10^{\circ}$ in Fig. 23 (left) and $\alpha=15^{\circ}$ in Fig. 23 (right). For comparison, the experimental data of Gregory and O'Reilly ${ }^{23}$ are used, which are provided on the TMR Website ${ }^{7}$ and are considered most suitable, although they were obtained at a lower Reynolds number of $R_{c}=3 \cdot 10^{6}$ based on the chord length of the airfoil. On the overall scale there is good agreement of all numerical predictions with the experimental $C_{p}$ data, as shown in Fig. 23, whereas some differences can be observed when enlarging the suction peak region, as shown in Fig. 24. Clearly, around and downstream of the suction peak, the SA model tends to predict slightly lower pressure than the SST and the SSG/LRR- $\omega$ model. Even if there is still some grid influence, as can be concluded from the difference between the CFL3D and TAU results with the SSG/LRR- $\omega$ model, at $\alpha=15^{\circ}$, this influence is obviously smaller than the difference between the SA and the SSG/LRR- $\omega$ predictions.

However, in view of the experimental resolution and the uncertainty with respect to Reynolds number effects, any further assessment of the model predictions is impossible. All one can say is that all turbulence models seem to give reasonable results for this flow, where the SSG/LRR- $\omega$ and SST model predictions are very similar to one another and show small differences from the SA model results.

\section{IV.A.3. Axisymmetric separated boundary layer}

The prediction of flow separation is a major challenge for any RANS turbulence model. As one representative of this class, the TMR Website ${ }^{7}$ provides meshes and experimental data for the subsonic flow along a cylinder subjected to a pressure gradient by varying the contour of the surrounding outer streamline shape. The advantage of axi-symmetric configurations over two-dimensional configurations is that there are no possible side-wall effects that might spoil the flow. The flow has been studied experimentally by Driver ${ }^{24}$ The flow conditions are given by a Mach number of $M=0.08812$ and a Reynolds number of $\operatorname{Re}_{L}=2 \cdot 10^{6}$ based on unit length of $L=1 \mathrm{~m}$. The reference temperature used is $T_{R e f}=288.9 \mathrm{~K}$.

Numerical simulations have been performed using CFL3D and TAU on the second finest structured mesh with $N=713 \times 193$ points provided on the TMR Website. ${ }^{7}$ In the simulations, the pressure gradient is generated by a streamline shaped outer wall with inviscid boundary conditions, where the deviation from the straight cylindrical wall extends from $x \approx-0.3 m$ to $x \approx 0.85 m$ with the maximum deflection at $x \approx 0.3 m$. The grid structure and the employed boundary conditions are shown in Fig. $25{ }^{7}$

Figure 26 (left) shows the predicted pressure distributions compared to the experimental data along the cylinder surface in the region of the varying pressure gradient. Note that all results have been shifted in order to match $C_{p}=0$ at $x=-0.4 \mathrm{~m}$. All models overestimate the pressure rise, where the SA model shows the largest deviation from the experimental data. Similar to the previous cases, the SST and SSG/LRR- $\omega$ predictions are very similar to one another. Also, TAU was run for this case with both first and second order turbulence advection terms. The higher order treatment makes very little difference in the results.

The predicted distributions of the local skin friction coefficient, $c_{f}$, along the surface are shown in Fig. 26 (right). Indicated by $c_{f}<0$, the SA model predicts a comparatively large region of separated flow, which is consistent with the predicted larger maximum pressure coefficient values, i.e., a larger overall adverse pressure gradient. The SST model predicts a slightly smaller region of separated flow, which appears to be

10 of 47

American Institute of Aeronautics and Astronautics 
in fair agreement with the experimental data. The SSG/LRR- $\omega$ model, although yielding almost the same pressure distribution as the SST model, predicts only a very small region of separated flow that is hardly visible on this scale.

Figure 27 illustrates the flow fields predicted by the SSG/LRR- $\omega$ model (left), the SA model (center) and the SST model (right) by depicting the corresponding streamlines in the region of the flow separation. Note that for clarity the wall normal $y$-axis is enlarged. With the SSG/LRR- $\omega$ model, the separation bubble is too shallow to be visible, whereas the SA and the SST models yield larger bubbles that can easily be identified. As will be shown in the velocity profiles, the larger bubbles are closer to the experiment.

Figure 28 shows the corresponding profiles of the non-dimensional streamwise velocity component normalized with the inflow velocity, $U_{\infty}$, at six different positions in the region of the adverse pressure gradient. Clearly, at the most downstream position at $x=-0.0762 \mathrm{~m}$ there is little difference between the predictions, and all models apparently agree well with the experimental data. Nevertheless, consistent with the observations on the pressure distribution, the SA model slightly underestimates the boundary layer edge velocity. In all positions further downstream, the SST model predictions maintain good agreement with the experiment, while in particular the SSG/LRR- $\omega$ model predictions deviate from the experimental data close to the wall. Some very small differences between the CFL3D and the TAU results can be observed in the two most downstream positions, but the characteristics of the solution remain the same.

Figure 29 shows the profiles of the non-dimensional Reynolds shear stress component, $R_{x y}$, scaled with the square of the inflow velocity, $U_{\infty}^{2}$, at the same six positions. At the three upstream positions, the SA model overpredicts the Reynolds shear stress, while it underpredicts it at the two most downstream positions. (Again, some differences between the SSG/LRR- $\omega$ results of CFL3D and TAU can be seen at the two most downstream positions, but they are relatively minor.) In contrast, the SSG/LRR- $\omega$ model underpredicts the Reynolds shear stress at the three upstream positions and is in fair agreement with the experiment at the two most downstream positions. The SST model shows good agreement with the measurement at virtually all positions, except the last one where it underpredicts the Reynolds shear stress.

Thus, predicted large flow separation appears to be accompanied by high upstream and low downstream Reynolds shear stress, and vice versa when only little separation is predicted. Overall the SST model clearly gives the best predictions for this case, while the small sensitivity of the SSG/LRR- $\omega$ model to separation is a bit disappointing.

\section{IV.A.4. Transonic flow over an axisymmetric bump}

In transonic flow, shock induced separation may occur. As a representative of this phenomenon, the transonic flow along a cylinder with a bump shaped as a circular arc has been studied experimentally by Bachalo and Johnson. ${ }^{25}$ The Mach number is $M=0.875$ and the Reynolds number is $R e_{c}=2.763 \cdot 10^{6}$ based on the chord length, $c$, of the bump. The reference temperature is taken to be $T_{R e f}=300 \mathrm{~K}$. Corresponding grids and experimental data are provided on the TMR Website, ${ }^{7}$ where the grid structure and boundary conditions are sketched in Fig. 30. ${ }^{7}$ The bump is located between $0 \leq x / c \leq 1$, so that its crest is located at $x / c=0.5$. Numerical simulations have been performed on the second finest structured mesh with $N=721 \times 321$ points.

Figure 31 (left) shows the predicted surface pressure distributions in the range $x / c=0.4$ to $x / c=1.7$, covering the region from just upstream of the bump crest to 0.7 chord lengths downstream of the trailing edge of the bump. The experimental data show a suction peak at $x / c \approx 0.62$ followed by a steep increase in pressure, indicating a shock. The SA model slightly overestimates the suction peak and predicts the shock somewhat downstream of the experimental position, whereas the SST and particularly the SSG/LRR- $\omega$ model predictions are in closer agreement with the experimental data. Downstream of the position $x / c \approx$ 0.75 , all models tend to overpredict the pressure, where the SST model shows the smallest and the SA model the largest deviation. Downstream of the position $x / c \approx 1.4$, all models predict a similar pressure coefficient, $C_{p}$, in fair agreement with the experiment. Note that there is very little difference between the CFL3D and TAU predictions with the SSG/LRR- $\omega$ model.

Although no experimental data are available, the predicted skin friction distributions in terms of $c_{f}$ versus $x / c$ are plotted in Fig. 31 (right). Around the leading edge of the bump, the SA model predicts the lowest skin friction, whereas just upstream of the shock it predicts the highest $c_{f}$ of all models. The SST and the SSG/LRR- $\omega$ models yield very similar skin friction upstream of $x / c \approx 0.1$, whereas further downstream the SST model predicts lower $c_{f}$ values that up to the shock position are the lowest of all models. In the experiment, shock induced separation starts at $x / c \approx 0.7$, slightly downstream of the shock position observed 
in the pressure distribution. ${ }^{25}$ According to Bachalo and Johnson, the flow reattaches at $x / c \approx 1.1 .{ }^{25}$ Both experimental separation and reattachment positions are indicated in the figure.

Figure 31 (right) shows that all models predict separation onset directly behind the shock, indicated by the steep drop in $c_{f}$, whereas in the experiment there is some distance in between the shock and separation. Consequently, the SA model predicts the position of separation onset closest to the experiment and SST the furthest upstream of the experimental position. Both SA and SST predict the reattachment downstream of the position observed in the experiment, leading to an overestimation of the length of the separation. In contrast, the SSG/LRR- $\omega$ model predicts both separation onset and reattachment slightly upstream of the respective experimental positions, leading to an overall good agreement with the experimental separation length. Nevertheless, the model predicts the highest $c_{f}$ levels within the separated region.

Figure 32 shows the corresponding streamline pattern as predicted by CFL3D with the different models. The bubbles vary considerably in length, and the SSG/LRR- $\omega$ model predicts a more rapid change in flow direction towards reattachment than the SA and the SST models. This behavior is probably related to the non-physical back-bending of streamlines observed by Jakirlić and Maduta with their model, ${ }^{26}$ indicating that the SSG/LRR- $\omega$ model might benefit from a correction similar to the one described in Ref. 26 . Note that despite the differences in $c_{f}$, the SSG/LRR- $\omega$ predicts a bubble of similar thickness as the SA model, whereas the SST model not only yields the longest, but also the widest bubble. This observation is confirmed when looking at the profiles of the non-dimensional streamwise velocity, $U$, normalized with the freestream velocity, $U_{\infty}$, at six different streamwise positions, shown in Fig. 33. Upstream of the bump, at $x / c=-0.25$, all models predict a similar velocity profile in good agreement with the experimental data. At $x / c=0.688$, in between the experimental shock position and separation onset, large deviations are observed between the different model predictions and the experiment. Close to the wall, the SA model result shows the best agreement with the measurement, whereas towards the boundary layer edge the SSG/LRR- $\omega$ model result is closest to the experiment. Nevertheless there is some deviation also with the SSG/LRR- $\omega$ model. Note the difference between the CFL3D and the TAU result at $x / c=0.688$, which is due to high sensitivity in the predicted shock position.

At positions $x / c=0.813$ and $x / c=1.000$, within the experimentally observed separation bubble, the SSG/LRR- $\omega$ model underestimates the reverse flow velocity, whereas the SST model prediction is in good agreement with the measurement and the SA model slightly overestimates it. Outside the bubble, the SA model shows the best agreement with the experimental velocity profiles, which holds for all positions $x / c \geq 0.813$.

Note that at $x / c=1.000$, the SSG/LRR- $\omega$ and the SA model yield zero streamwise velocity at almost the same position, indicating a similar thickness of the separation bubble. Apparently, the smaller absolute value in $c_{f}$ observed with the SSG/LRR- $\omega$ is caused by a too low reverse flow speed inside the bubble, not because of a too small size of the separation.

At the positions $x / c=1.125$ and $x / c=1.375$, downstream of the experimental reattachment point, the SSG/LRR- $\omega$ model predicts the highest velocity near the wall, which is closest to the experimental data. However, this might be a consequence of its prediction of a relatively small normal extent of the separation bubble. Note that the predicted velocity profiles obtained with the SSG/LRR- $\omega$ model and the SST model are very similar, except near the wall, including the separation. The SST limiter, designed to be active near the wall for adverse pressure gradient flows, ${ }^{4}$ is likely the main reason for the difference.

Figure 34 shows the corresponding profiles of the non-dimensional Reynolds shear stress, $R_{x y}$, normalized with the squared freestream velocity, $U_{\infty}^{2}$, at the same six streamwise positions as the above velocity profiles. Similar to the velocity predictions, upstream of the bump at $x / c=-0.25$, all model predictions are similar and agree well with the experimental data. At $x / c=0.688$, all models show some deviations from the experiment, which can probably be attributed to the vicinity to the shock that is predicted at slightly different positions. At all positions further downstream, $x / c \geq 0.813$, the SSG/LRR- $\omega$ model always predicts the highest and the SA model the lowest Reynolds stresses, where the SSG/LRR- $\omega$ result is in particularly good agreement at $x / c=0.813$ (in the separation bubble) and at $x / c=1.1375$ (downstream of the reattachment point). At $x / c=1.000$, which is still in the separation bubble, and at $x / c=1.125$, which is in the immediate vicinity of the experimental reattachment point, all models underpredict the measured Reynolds shear stress.

An assessment of models with respect to this test case appears to be particularly difficult because of two effects. First, the shock, as the cause of the flow separation, needs to be predicted at the correct position and with correct strength. Second, the model needs to react correctly to the shock, i.e., to the strong adverse pressure gradient in terms of the size of the separated region and accompanying details of the flow field, as 
well as to any relaxation effects downstream of the shock. All models show certain advantages, but only with respect to part of the flow characteristics.

The SA model predicts the shock downstream of the experimental position, thus the shock is stronger and causes a comparatively large separation bubble. Separation onset is predicted in good agreement with the experiment, but the bubble appears too long. This is accompanied by an underestimation of the Reynolds shear stress, but, except for the near-wall region, the velocity profiles agree well with the measured ones.

The SST model apparently predicts the shock slightly upstream of the experimental position, causing separation onset upstream of the experimental position. The separation bubble is shorter than predicted by the SA model, but reattachment still occurs downstream of the experimental position. There is good agreement with the experimental reverse flow velocity profiles within the separation bubble, but there are larger deviations outside. The Reynolds shear stress is predicted larger than by the SA model, but it still underestimates the levels found in the experiment.

The SSG/LRR- $\omega$ model predicts the shock position in very good agreement with the experiment, but separation onset is found upstream of the measured position. The separation length appears to be in good agreement with the experiment, but this means that the reattachment point is also predicted upstream of the experimental position. Except for two positions, the Reynolds shear stress is predicted in rather good agreement with the measurements and is generally closest to the experiment compared to the other models. The thickness of the bubble is predicted similar to the SA model, but the reverse flow speed inside the bubble is underestimated. In the outer part of the boundary layer, the velocity profiles show similar deviations as with the SST model.

\section{IV.A.5. Summary of Basic Validation Cases}

For the flow over a flat plate at zero pressure gradient and the flow around a NACA0012 airfoil up to $\alpha=15^{\circ}$, where the flow is mainly attached, all models yield similar results in good agreement with theoretical results and experimental data, respectively. In the case of the separating axisymmetric boundary layer flow, the SSG/LRR- $\omega$ underpredicts the separation extent. For the transonic flow over an axisymmetric bump, the SSG/LRR- $\omega$ model predicts the shock location and the length of the separation bubble in (very) good agreement with the experiment, but the reverse flow speed inside the bubble is underestimated.

\section{IV.B. Extended Validation Cases}

\section{IV.B.1. Subsonic Flow around the NACA4412 Airfoil at $\alpha=13.87^{\circ}$}

The subsonic flow around the NACA4412 airfoil at an incidence of $\alpha=13.87^{\circ}$ can be viewed as a simple case representative of high-lift flow, involving separation near the trailing edge. The flow has been studied experimentally by Coles and Wadcock ${ }^{27,28}$ at a comparatively low Reynolds number of $R e_{c}=1.52 \cdot 10^{6}$ based on the chord length $c$, where transition has been tripped at $x / c=0.025$ on the upper surface and at $x / c=0.103$ on the lower surface. ${ }^{7}$

In contrast, according to the TMR Website, ${ }^{7}$ the numerical simulations are carried out in fully turbulent mode on the second finest structured mesh with $N=897 \times 257$ points also provided on the TMR Website. ${ }^{7}$ As depicted in Fig. $35,{ }^{7}$ the far field is located approximately 100 chord lengths away from the airfoil. Mach number is $M=0.09$ and reference temperature is $T_{R e f}=297.8 \mathrm{~K}$.

Note that the NACA4412 experiment was in a relatively small wind tunnel, which likely had some influence. As a result, as discussed on the TMR website, ${ }^{7}$ there are some uncertainties associated with running the case "correctly" vis-a-vis the experiment. Including the wind-tunnel walls might remove part of the uncertainties, but would considerably increase the numerical effort and probably raise questions regarding the prediction of secondary flow. Despite the uncertainties, this case can still be instructive for comparing different models' behaviors for airfoil trailing edge separation.

Note also that with this particular test case, a difference between CFL3D, FUN3D and TAU has been observed, when running the SSG/LRR- $\omega$ model. While with TAU, after some initial convergence problems, a steady state solution has been found, with CFL3D and FUN3D the solution remains unsteady, even when running a time accurate simulation. With a more recent version of TAU, a different researcher also could not obtain a steady state solution for this case when starting from freestream conditions. However, when restarting from a solution at $\alpha=10^{\circ}$, for which a steady state solution can be found, and successively increasing the incidence, the steady state solution at $\alpha=13.87^{\circ}$ has been confirmed. This might be an indication of the existence of two different solutions, one being steady and one unsteady. Therefore, only 
steady state TAU results for SSG/LRR- $\omega$ and steady CFL3D results for SA and SST are presented, and the steady state TAU solution needs to be considered with some caution.

Figure 36 (left) shows the pressure distributions predicted with TAU, using the SSG/LRR- $\omega$ model, and with CFL3D, using the SA and the SST models compared to the experimental data by Coles and Wadcock. ${ }^{27,28}$ The SA model predicts the highest suction peak and lower pressure than the SST and the SSG/LRR- $\omega$ models all the way to $x / c \approx 0.8$ on the suction side, apparently in better agreement with the experimental data. However, the experimental data are uncorrected for wind tunnel wall effects and, according to the TMR Website, should be viewed in a qualitative sense, only. ${ }^{7}$

Figure 36 (right) shows the distribution of the local skin friction coefficient, $c_{f}$, near the trailing edge as numerically predicted with the different models. As indicated by negative $c_{f}$ values, all models predict separation at almost the same position, i.e., the SST model at $x / c \approx 0.775$, the SSG/LRR- $\omega$ model at $x / c \approx 0.78$ and the SA model at $x / c \approx 0.79$. Interestingly, the SA and SST models yield almost identical values for the negative skin friction coefficient in the separation region that are almost twice as large in magnitude as the values predicted by the SSG/LRR- $\omega$ model.

Note that the $c_{f}$ level in the reverse flow region must not be misinterpreted as a measure of the thickness of the separation bubble, as becomes clear from the streamline patterns depicted in Fig. 37. Indeed, the SST model predicts the largest separation bubble of all models tested. However, despite the different $c_{f}$ level, the SSG/LRR- $\omega$ model predicts a separation bubble of similar thickness that encompasses a smaller region downstream of the trailing edge than SST. In contrast, the SA model predicts a separated region that is clearly shallower, although $c_{f}$ is nearly identical to the SST model result.

Figure 38 shows computed and measured profiles of the non-dimensional streamwise velocity component, $U$, along the wall normal direction at six different positions on the upper surface. Note that an unusual reference velocity, $U_{R e f} \approx 0.93 U_{\infty}$, is used in the nondimensionalization of velocity and Reynolds stress. See the TMR website ${ }^{7}$ for more details. Different from the results for the pressure coefficient, the SA model shows considerable deviation from the experimental data, predicting a too shallow separation bubble, as indicated by the region of negative streamwise velocity. In contrast, the predictions of the SST and the SSG/LRR- $\omega$ models both agree quite well with the measured velocity profiles, where the SST model yields higher maximum reverse streamwise velocity. Note that, in particular at $x / c=0.8973$ and at $x / c=0.9528$, the SSG/LRR- $\omega$ model yields the smallest and the SA model the largest absolute values of the streamwise velocity component in the reverse flow region, indicating that the flow in the bubble is moving at different speeds. This is assumed to be the major reason for the fact that the SSG/LRR- $\omega$ model yields smaller absolute values of $c_{f}$ than the SST model, although the predicted bubble sizes are apparently similar.

Figure 39 shows the corresponding profiles of the non-dimensional Reynolds stress component $R_{x y}$. All models underestimate the experimental values, where the SA model shows the largest and SSG/LRR- $\omega$ model the smallest deviation from the measurement. Nonetheless, the latter difference still appears considerable.

\section{IV.B.2. Flow Through a Curved Duct}

The subsonic flow through a rectangular duct, undergoing a rapid $30^{\circ}$ bend, addresses the effects of strong streamline curvature compared to the boundary layer thickness. The flow has been studied experimentally by Smits et al. ${ }^{29}$ in a duct with a height of $H=0.127 \mathrm{~m}$ and an aspect ratio of $6: 1$, where the focus is on the boundary layer on the convex (lower) wall. According to the TMR Website, ${ }^{7}$ the flow conditions are defined by a subsonic Mach number of $M=0.093$ and a Reynolds number of $R e_{H}=2.667 \cdot 10^{5}$ based on the duct height $H$. Reference temperature is $T_{R e f}=293.3 \mathrm{~K}$. On the (upper) concave wall, considerable spanwise variation of the skin friction has been observed, indicating the presence of longitudinal Görtler vortices that may develop due to centrifugal forces. However, for the purposes of validation, the influence of any three-dimensionality along the upper wall is assumed to have minimal influence on the flow very near the convex surface, and all computations are performed in 2-D.

The numerical predictions have been carried out on the second finest structured mesh with $N=513 \times$ 193 points provided on the TMR Website. ${ }^{7}$ Figure 40 (left) shows the overall geometry together with the corresponding boundary conditions, while Fig. 40 (right) defines the coordinates used upstream and downstream of the bend. Both figures are taken from the TMR Website. ${ }^{7}$

Figure 41 shows the pressure distributions predicted by the different models compared to the experimental data. Note that all numerical results for the pressure coefficient, $C_{p}$, have been shifted by $\Delta C_{p}=-0.04$, in order to match the experimental reference. ${ }^{7}$ Apparently there is little difference between the model predictions, all agreeing well with the experimental data. 
Larger differences are observed in the distribution of the skin friction coefficient $c_{f}$ on the lower convex wall, Fig. 42 (left). The SSG/LRR- $\omega$ result follows the admittedly sparse experimental data quite closely, whereas the SA model considerably overestimates the local minimum just downstream the bend. The SST model matches the minimum in $c_{f}$, but predicts a slightly stronger increase downstream. In contrast, on the upper concave wall, the SSG/LRR- $\omega$ model predicts the highest skin friction of all models, as shown in Fig. 42 (right). Nevertheless the $c_{f}$ level present in the experiment is underestimated by all models. However, it should be noted that the simulations are carried out in two-dimensions only, preventing any Görtler vortices from being represented.

Figure 43 shows the profiles of the non-dimensional wall-parallel velocity component, scaled with the experimental reference velocity of $U_{R e f}=31.9 \mathrm{~m} / \mathrm{s}$, at six different stations along the lower convex wall, where $x=0$ refers to the end of the bend. As one can see, upstream of the bend at $x=-0.166 \mathrm{~m}$, all model predictions agree well with the experimental data, whereas directly downstream of the bend at $x=0.030 \mathrm{~m}$, all models overestimate the wall parallel velocity. Further downstream, the differences become smaller again, where at $x=0.183 \mathrm{~m}$ and $x=0.335 \mathrm{~m}$ the SSG/LRR- $\omega$ model predictions appear to be in very good agreement with the experiment, while the SA and SST model predictions show some deviations. For the two most downstream positions at $x=0.635 \mathrm{~m}$ and $x=1.250 \mathrm{~m}$ a definite assessment is more difficult, since all model predictions show some deviations from the experiment.

Concerning the corresponding Reynolds shear stress component termed $R_{x y}$ throughout, the SSG/LRR$\omega$ model predictions are in rather good agreement with the experiment, only overestimating the minimum value at $x=0.355 \mathrm{~m}$ and $x=0.635 \mathrm{~m}$. At these positions, the SA and the SST models predict the minimum level in closer agreement with the experiment, but show larger deviations from the experiment in every other respect.

Thus, there appear to be some advantages of the SSG/LRR- $\omega$ model for this kind of flow. Nevertheless, note that SA and SST predictions can be improved for this case by employing some rotational or curvature correction, ${ }^{7}$ which, however, should then be tested with other validation cases as well.

\section{IV.B.3. Two-dimensional Flow Over a Wall-Mounted Hump}

The two-dimensional flow over a wall-mounted hump has been used for studying both separation and flow control. It has been investigated experimentally by Greenblatt et al., ${ }^{30-32}$ at an inflow Mach number of $M=0.1$ and a Reynolds number of $R e_{c}=0.936 \cdot 10^{6}$ based on the chord length of the hump, and served as a test case of the NASA CFDVAL2004 Workshop. ${ }^{33}$ Reference temperature is $T_{R e f}=298.3 \mathrm{~K}$.

On the TMR Website, ${ }^{7}$ the case is presented without any flow control in order to assess turbulence models' capabilities to predict separation. Nevertheless, the geometry contains the plenum chamber inside the hump. The numerical predictions have been carried out on the "fine" structured mesh with $N=210,060$ points provided on the TMR Website, ${ }^{7}$ which is identical to one of the grids provided in the original NASA CFDVAL2004 Workshop. The geometry and corresponding boundary conditions are shown in Fig. $45 .{ }^{7}$ The hump leading edge is located at $x / c=0$.

Note that for this particular test case, only results with CFL3D and FUN3D are presented, as considerable differences have been observed between the TAU and the CFL3D and FUN3D SSG/LRR- $\omega$ results to date. Additional tests with TAU revealed a sensitivity of the solution to a variation in the outlet boundary condition; however, the reason for the differences has not yet been identified. Therefore the TAU results have been omitted.

Figure 46 (left) shows the computed distributions of the local pressure coefficient, $C_{p}$, along the wall compared to the experimental data by Greenblatt et al. ${ }^{30-32}$ Note that all simulation data have been shifted by $\Delta C_{p}=-0.015$, in order to better match the experimental reference upstream. All models underpredict the suction peak at $x / c \approx 0.5$. Further downstream, the experimental data show a local maximum in $C_{p}$ at $x / c \approx 0.7$, followed by another small suction peak at $x / c \approx 0.85$. Apparently, none of the models can represent this behavior in close agreement with the measurement. The SA model gives the correct shape, but overpredicts the local pressure maximum at $x / c \approx 0.7$. In contrast, the SST and the SSG/LRR- $\omega$ models predict the local pressure maximum in good agreement with the experiment, but in particular the SSG/LRR- $\omega$ fails to predict the second suction peak. Downstream of the second suction peak, the SA model is in fair agreement with the experimental data, whereas the SST and the SSG/LRR- $\omega$ models show larger deviations. Note that there is no noticeable difference between the CFL3D and the FUN3D results with the SSG/LRR- $\omega$ model. 
Obviously, the behavior in the range $0.7 \leq x / c \leq 1.2$ is related to flow separation, as indicated in Fig. 46 by the region of negative skin friction coefficient, $c_{f}$. The SSG/LRR- $\omega$ model predicts the streamwise extent of the separation bubble in fair agreement with the experiment and consequently shows the least deviations in the downstream recovery region. However, it underestimates the $c_{f}$ levels in the separation bubble that are predicted by the SST and the SA models in much better agreement with the experiment. In contrast, the SA and SST models overestimate the streamwise extent of the separation and show larger deviations in the recovery region.

Figure 47 illustrates the predicted flow fields in terms of the respective streamline patterns. As indicated by the skin friction distribution, the SSG/LRR- $\omega$ model predicts the shortest bubble, but apparently also the widest one. As with the transonic axisymmetric bump case, the SSG/LRR- $\omega$ model predicts bubble reattachment with a more rapid change in flow direction (i.e., "back-bending," as discussed in section IV.A.4). The SA and the SST models predict a longer and, in particular the SA model, a shallower bubble. Apparently, the low absolute value of the skin friction coefficient in the separation region predicted by the SSG/LRR- $\omega$ model is an indication of too low reverse flow speed, but is not an indication of too little separation. Thus, in this case, the apparently improved agreement with the experimental $c_{f}$ data near to and downstream of the reattachment point should probably not be interpreted as a compensation for insensitivity of the model to separation.

This is confirmed when considering the velocity profiles. Figure 48 shows profiles of the non-dimensional streamwise velocity component, $U$, scaled with the reference velocity, $U_{R e f}$, at six different stations along the bubble, beginning at $x / c=0.80$, where the flow is already separated. Clearly, at the three positions $x / c=0.80, x / c=0.90$ and $x / c=1.00$, the SSG/LRR- $\omega$ model underestimates the reverse flow velocity, and shows the widest region of reverse flow. Apparently, it is the too-small reverse flow speed that is responsible for the too low absolute values of $c_{f}$ observed in Fig. 46. The SST model shows the best agreement with the measured data of the reverse streamwise flow component, but also considerably overestimates the width of the reverse flow region. The SA model tends to overestimate the maximum reverse flow speed, but at these three positions is in very good overall agreement with the experimental data. In this respect, the situation resembles the observation with the separated axisymmetric boundary layer in section IV.A.3. One has to conclude that the experimental separation bubble is indeed shallower than predicted by the SSG/LRR- $\omega$ and the SST models. Note that the CFL3D and FUN3D results with the SSG/LRR- $\omega$ model predict nearly identical velocity profiles.

Figure 49 shows the corresponding profiles of the non-dimensional Reynolds stress component, $R_{x y}$, scaled with the square of the reference velocity, $U_{R e f}^{2}$. All models tend to underestimate the level of the Reynolds stress $R_{x y}$, where, except at position $x / c=0.80$, the SSG/LRR- $\omega$ gives the highest negative values. Apparently, higher upstream Reynolds stress levels are associated with a shorter separation bubble, but there is no obvious relation to its width.

In the two most downstream positions, the SSG/LRR- $\omega$ model predicts the profiles of the non-dimensional Reynolds stress component, $R_{x y} / U_{R e f}^{2}$, in fair agreement with the experimental data. This is probably a consequence of the more upstream reattachment point, which, according to the distribution of the skin friction coefficient $c_{f}$ shown in Fig. 46 (right), is in closer agreement with the experiment than with the other models.

The differences between the CFL3D and FUN3D results with the SSG/LRR- $\omega$ are somewhat larger for the non-dimensional Reynolds stress component, $R_{x y} / U_{R e f}^{2}$, than for the other quantities shown. Nevertheless they are clearly smaller than the differences between the results for different models.

Thus, all models show a combination of advantages and disadvantages. The SSG/LRR- $\omega$ model predicts a separation bubble that has approximately the correct length, but is too thick, and the reverse flow speed inside the bubble is too low. The SST model predicts a separation bubble that is too wide and too long, whereas the reverse flow speed agrees with the experiment. The SA model predicts a separation bubble that probably has the correct width, but is too long and the reverse flow speed is higher than in the experiment. Furthermore, there appears to be only a relation between the predicted level of the Reynolds stress component $R_{x y}$ and the predicted length of the separation bubble, but not with its width.

\section{IV.B.4. Supersonic flow Through a Square Duct}

In a duct with rectangular cross section, secondary flows develop longitudinal vortices that are caused by the difference between the Reynolds normal stresses close to walls. This phenomenon cannot be represented by linear eddy-viscosity models due to the underlying Boussinesq hypothesis. 
The corresponding test case on the TMR Website ${ }^{7}$ involves the supersonic flow at a Mach number of $M=3.9$ through a square duct of height and width $D=25.7 \mathrm{~mm}$ over a length of $L=50 D$. The Reynolds number is $R e_{D}=5.08 \cdot 10^{5}$ based on the duct height $D$. Reference temperature is taken to be $T_{R e f}=288.9$ K. The flow has been studied experimentally by Davis and Gessner. ${ }^{34}$ Figure 51 (left) shows an overall sketch of the geometry together with the corresponding boundary conditions. Figure 51 (right) shows the grid structure in a cross-section of the duct. Both figures are taken from the TMR Website. ${ }^{7}$ Only one quarter of the duct is simulated.

The numerical predictions are typically carried out on the second finest structured mesh provided on the TMR Website ${ }^{7}$ with $N=481 \times 81 \times 81$ points. However, for this case it was found that the deviations between the predictions of CFL3D and TAU on the second finest grid using the SSG/LRR- $\omega$ are larger than observed with the other test cases shown previously. For this reason, FUN3D results have been included, confirming the larger spread. These differences indicate a larger grid influence with the SSG/LRR- $\omega$ than with the eddy-viscosity models for this case. This is also confirmed by computations with TAU on the finest grid with $N=961 \times 161 \times 161$ points.

Figure 51 shows the distribution of the local skin friction coefficient along the vertical wall at $x / D=50$ as predicted by the different models compared to the experimental data. Most of the CFD results are from the second-finest grid, but the TAU results are also plotted for SSG/LRR- $\omega$ on the finest grid. There is some small change due to grid refinement. The SSG/LRR- $\omega$ model yields the skin friction coefficient in fair agreement with the experimental data, except for the duct center. In contrast, the SA and SST models results show large deviations throughout, which can be attributed to the missing secondary flow they cannot predict.

Figures 52 and 53 show the numerical results for the non-dimensional streamwise velocity component along the vertical centerline (left) and along the diagonal (right) at $x / H=40$ and 50 compared to the respective experimental data. The SSG/LRR- $\omega$ model predicts profiles in fair agreement with the measurement. In contrast, the SA and the SST model tend to overpredict the non-dimensional streamwise velocity along the vertical centerline, whereas they underpredict it along the diagonal. As already stated, this is expected due to the general inability of linear eddy-viscosity models to predict the secondary flow.

Figure 54 shows the velocity contours in the cross-section at $x / H=40$ as predicted by the three codes, using the SSG/LRR- $\omega$ model. The two counter-rotating longitudinal vortices are clearly visible. Although not shown here, the linear eddy-viscosity models show no indication of these structures. For comparison, the TAU results on the finest grid are also depicted, illustrating that the results for the SSG/LRR- $\omega$ model on the second finest mesh are not yet grid converged.

\section{IV.B.5. Summary of Extended Validation Cases}

For the flow around the NACA4412 airfoil, the SSG/LRR- $\omega$ model predicts the trailing-edge separation similar to the SST model, but yields a lower reverse flow velocity. For the flow over a wall-mounted hump, the SSG/LRR- $\omega$ model predicts the length of the separation bubble in fair agreement with the experimental data, but apparently overestimates its width. The reverse flow velocity inside the bubble is again underestimated. For the curved duct case the SSG/LRR- $\omega$ model yields the best results of all models, where in particular the velocity profiles downstream of the bend are in very good agreement with the experiment. For the supersonic flow through a square duct, the SSG/LRR- $\omega$ model predicts secondary flow and shows fair agreement with the experimental skin friction and velocity data in two downstream cross-sections. As expected, for the latter two flow cases, the SSG/LRR- $\omega$ model is superior to the SA and the SST models.

\section{Conclusion}

The SSG/LRR- $\omega$ differential Reynolds stress model has been implemented into the flow solvers CFL3D and FUN3D by NASA and into TAU by DLR. The respective implementations have been cross-checked by running three verification test cases of the TMR Website ${ }^{7}$ on a series of successively refined structured grids: the flow over a flat plate with zero pressure gradient, a plane jet flow and the two-dimensional flow over a hump. The development of characteristic integral and local quantities with increasing mesh resolution has been checked, showing that the different results apparently converge to a common solution. Excellent agreement is found, comparing the pressure and the skin friction distribution as well as velocity and Reynolds stress profiles obtained on the respective finest grid, giving confidence in the correctness of 
the model implementation into the different codes.

A series of four basic validation test cases has been studied running CFL3D and TAU with the SSG/LRR$\omega$ model: the flow over a flat plate at zero pressure gradient, the flow around the NACA0012 airfoil at different angles of attack, an axisymmetric separating boundary layer flow and the transonic flow over an axisymmetric bump. The results have been compared against experimental data as well as simulation data obtained with CFL3D, using the SA and the SST models.

For the flat plate flow, all results are very similar and in good agreement with experimental skin friction data and the log-law of the velocity profile. It is noted that the SSG/LRR- $\omega$ and the SST model results are almost identical. The SA model predicts slightly higher skin friction, which is probably associated with a slightly smaller boundary layer thickness, $\delta$, and displacement thickness $\delta^{*}$ at the same location.

Good agreement between the models is also found with the NACA0012 case, where, only at $\alpha=15^{\circ}$, the SSG/LRR- $\omega$ and the SST models yield slightly lower lift and higher drag than the SA model. Apparently this is related to a higher suction peak predicted by the SA model.

Similarly, for the separated axisymmetric boundary layer case the SSG/LRR- $\omega$ and the SST models predict a smaller pressure rise in better agreement with the experiment than the SA model. Nevertheless, the SSG/LRR- $\omega$ model significantly underestimates the associated separation, whereas the SST model predicts the flow characteristics in good agreement with the experimental data. With the SA model, the length of the separation is slightly overpredicted, but the velocity profiles are in good agreement with the measured ones.

The transonic flow over an axisymmetric bump shows the largest differences between the models and is the basic test case that is the most difficult to be assessed. The reason is that the models predict the shock at slightly different positions and thus, with different strength. The SSG/LRR- $\omega$ model predicts the shock position and the length of the separation bubble in good agreement with the experiment, but the reverse flow speed inside the bubble is underestimated. This is probably the cause for too low absolute values of the skin friction coefficient in the reverse flow region. The SST model predicts the shock slightly upstream, whereas the SA model predicts it downstream of the experimental position. Both models yield longer separation bubbles and larger absolute values of the skin friction coefficient in the reverse flow region. Downstream of the shock the SA model shows the overall best agreement with the experimental velocity profiles.

Finally, four different flows from the list of extended validation test cases on the TMR Website ${ }^{7}$ have been studied: separated flow over a NACA4412 airfoil, the flow through a curved duct, separated flow over a wall-mounted hump, and supersonic flow in a square duct.

The flow around the NACA4412 airfoil at incidence $\alpha=13.87^{\circ}$ involves a trailing-edge separation. This case is difficult to compute with SSG/LRR- $\omega$, and there is some indication of the existence of multiple solutions, one steady and one unsteady. The steady SSG/LRR- $\omega$ solution and the SST solution yield similarly large bubbles, where the velocity profiles indicate fair agreement with the experiment. In contrast, the bubble predicted by the SA model is smaller. Once again the smaller absolute value of the skin friction coefficient predicted by the SSG/LRR- $\omega$ model compared to the SST model appears to be due to a lower reverse flow speed in the bubble.

The flow through a curved duct addresses effects of large streamline curvature that cannot be expected to be represented well by any eddy-viscosity model. In contrast, the SSG/LRR- $\omega$ predicts the pressure and skin friction distributions and even profiles of the Reynolds shear stress in rather good agreement with the experiment.

The flow over a wall mounted hump again involves a large region of separated flow that is predicted by all models differently. The SSG/LRR- $\omega$ models predict the length of the bubble in fair agreement with the experiment, but its width appears too large. With the SA model, the bubble width is probably close to experiment, but reattachment is too far downstream. Finally, the SST model apparently overestimates both length and width of the separated region. The SSG/LRR- $\omega$ model yields the best agreement with respect to Reynolds stress profiles.

The supersonic flow through a square duct is characterized by secondary flow that cannot be represented by linear eddy-viscosity models. In contrast, the SSG/LRR- $\omega$ model predicts the associated longitudinal vortices and represents skin friction and velocity profiles in fair agreement with the experimental data.

To summarize: for a wide range of separated flows the SSG/LRR- $\omega$ model yields similar results as the SA or the SST models. Advantages have been observed with respect to the prediction of the shock position and the length of separation bubbles. However, the reverse flow speed inside the bubble seems to be generally underestimated. Furthermore, effects of streamline curvature and secondary flow are predicted 
reasonably well by the SSG/LRR- $\omega$ model. Capturing these effects requires curvature corrections or nonlinear extensions in standard eddy-viscosity models. Thus, the SSG/LRR- $\omega$ model appears to have a wider range of applicability than eddy-viscosity models without special modifications.

Future work on the SSG/LRR- $\omega$ model will be needed in order to improve the prediction of reverse flow speed in separation bubbles, and to eliminate the "back-bending" that occurs near bubble reattachment.

\section{Acknowledgments}

The authors are indebted to Dr. Mujeeb Malik and NASA's Revolutionary Computational Aerosciences program under the Transformational Tools and Technologies Project for fostering the collaboration between DLR and NASA.

\section{References}

${ }^{1}$ Spalart, P. R., "Strategies for Turbulence Modelling and Simulations," International Journal of Heat and Fluid Flow, Vol. 21, 2000, pp. 252-263.

${ }^{2}$ Wilcox, D. C., Turbulence Modeling for CFD, 3rd edition, DCW Industries, Inc., La Canada CA, 2006.

${ }^{3}$ Spalart, P. R. and Allmaras, S. R., "A One-Equation Turbulence Model for Aerodynamic Flows," Recherche Aèrospatiale, Vol. 1, 1994, pp. 5-21.

${ }^{4}$ Menter, F. R., "Two-Equation Eddy-Viscosity Turbulence Models for Engineering Applications," AIAA Journal, Vol. 32, No. 8, 1994, pp. 1598-1605.

${ }^{5}$ Hanjalić, K. and Launder, B., Modelling Turbulence in Engineering and the Environment, Cambridge University Press, 2012.

${ }^{6}$ Eisfeld, B. and Brodersen, O., "Advanced Turbulence Modelling and Stress Analysis for the DLR-F6 Configuration," AIAA Paper 2005-4727, Toronto, Canada, 2005.

${ }^{7}$ Rumsey, C. L., "Turbulence Modeling Resource," http://turbmodels.larc.nasa.gov, cited 13 April 2015.

${ }^{8}$ Haase, W., Aupoix, B., Bunge, U., and Schwamborn, D., "FLOMANIA - A European Initiative on Flow Physics Modelling," Notes on Numerical Fluid Mechanics and Multidisciplinary Design, Vol. 94, Springer, 2006.

${ }^{9}$ Speziale, C. G., Sarkar, and S. Gatski, T. B., "Modelling the Pressure-Strain Correlation of Turbulence: an Invariant Dynamical Systems Approach," Journal of Fluid Mechanics, Vol. 227, 1991, pp. 245-272.

${ }^{10}$ Shir, C. C., "A Preliminary Numerical Study of Atmospheric Turbulent Flows inn the Idealized Planetary Boundary Layer," Journal of the Atmospheric Sciences, Vol. 30, 1973, pp. 1327-1339.

${ }^{11}$ Daly, B. J. and Harlow, F. H., "Transport Equations of Turbulence," Physics of Fluids, Vol. 13, 1970, pp. $2634-2649$.

${ }^{12}$ Wilcox, D. C., "Reassessment of the Scale Determining Equation for Advanced Turbulence Models," AIAA Journal, Vol. 26, No. 11, 1988, pp. 1299-1310.

${ }^{13}$ Launder, B. E. and Sharma, B. I., "Application of the Energy-Dissipation Model of Turbulence to the Calculation of Flow Near a Spinning Disc," Letters in Heat and Mass Transfer, Vol. 1, 1974, pp. 131-138.

${ }^{14}$ Launder, B. E., Reece, G. J., and Rodi, W., "Progress in the Development of a Reynolds-Stress Turbulence Closure," Journal of Fluid Mechanics, Vol. 68, 1975, pp. 537-566.

${ }^{15}$ Wilcox, D. C., Turbulence Modeling for CFD, 2nd edition, DCW Industries, Inc., La Canada CA, 1998.

${ }^{16}$ Cécora, R.-D., Eisfeld, B., Probst, A., Crippa, S., and Radespiel, R., "Differential Reynolds Stress Modeling for Aeronautics," AIAA Paper 2012-0465, Nashville, TN, 2012.

${ }^{17}$ Hinze, J. O., Turbulence, 2nd edition, McGraw-Hill, 1975.

${ }^{18}$ Wieghardt, K., "Ueber die Wandschubspannung in turbulenten Reibungsschichten bei veränderlichem Außendruck," UM 6603, Kaiser-Wilhelm-Institut für Strömungsforschung, Göttingen, 1943.

${ }^{19}$ Spalart, P. R., "Philosophies and Fallacies in Turbulence Modeling," Progress in Aerospace Sciences, Vol. 74, 2015, pp. $1-15$.

${ }^{20}$ Coles, D. E. and Hirst, E. A., "Computation of Turbulent Boundary Layers - 1968 AFOSR-IFP-Stanford Conference, Vol. II, Compiled Data," 1969.

${ }^{21}$ Coles, D., "The Law of the Wake in the Turbulent Boundary Layer," Journal of Fluid Mechanics, Vol. 1, 1956, pp. 191-226.

${ }^{22}$ Ladson, C. L., "Effects of Independent Variation of Mach and Reynolds Numbers on the Low-Speed Aerodynamic Characteristics of the NACA 0012 Airfoil Section," NASA TM 4074, October 1988.

${ }^{23}$ Gregory, N. and O'Reilly, C. L., "Low-Speed Aerodynamic Characteristics of NACA 0012 Aerofoil Sections, including the Effects of Upper-Surface Roughness Simulation Hoar Frost," NASA R\&M 3726, January 1970.

${ }^{24}$ Driver, D. M., "Reynolds Shear Stress Measurements in a Separated Boundary Layer Flow," AIAA Paper 91-1787, Honolulu, HI, June 1991.

${ }^{25}$ Bachalo, W. D. and Johnson, D. A., "Transonic, Turbulent Boundary-Layer Separation Generated on an Axisymmetric Flow Model," AIAA Journal, Vol. 24, No. 3, 1986, pp. 437-443.

${ }^{26}$ Jakirlić, S. and Maduta, R., "Extending the Bounds of 'Steady' RANS Closures: Toward an Instability-Sensitive Reynolds Stress Model," International Journal of Heat and Fluid Flow, Vol. 51, 2015, pp. 175-194.

${ }^{27}$ Coles, D. and Wadcock, A. J., "Flying-Hot-Wire Study of Flow Past an NACA 4412 Airfoil at Maximum Lift," AIAA Journal, Vol. 17, No. 4, 1979, pp. 321-329. 

1979.

${ }^{29}$ Smits, A. J., Young, S. T. B., and Bradshaw, P., "The Effect of Short Regions of High Surface Curvature on Turbulent Boundary Layers," Journal of Fluid Mechanics, Vol. 94, Part 2, 1979, pp. 209-242.

${ }^{30}$ Greenblatt, D., Paschal, K. B., Yao, C.-S., Harris, J., Schaeffler, N. W., and Washburn, A. E., "A Separation Control CFD Validation Test Case, Part 1: Baseline and Steady Suction," AIAA Journal, Vol. 44, No. 12, 2006, pp. $2820-2830$.

${ }^{31}$ Greenblatt, D., Paschal, K. B., Yao, C.-S., and Harris, J., "A Separation Control CFD Validation Test Case, Part 2: Zero Efflux Oscillatory Blowing," AIAA Journal, Vol. 44, No. 12, 2006, pp. 2831-2845.

${ }^{32}$ Naughton, J. W., Viken, S. A., and Greenblatt, D., "Skin-Friction Measurements on the NASA Hump Model," AIAA Journal, Vol. 44, No. 6, 2006, pp. 1255-1265.

${ }^{33}$ NASA Langley Research Center Workshop "CFD Validation of Synthetic Jets and Turbulent Separation Control," March 29-31, 2004, Woodlands Hotel and Conference Center, Colonial Williamsburg, Williamsburg, Virginia, USA, http://cfdval2004.larc.nasa.gov/, cited 13 April 2015.

${ }^{34}$ Davis, D. O. and Gessner, F. B., "Further Experiments on Supersonic Turbulent Flow Development in a Square Duct," AIAA Journal, Vol. 27, No. 8, 1989, pp. 1023-1030. 


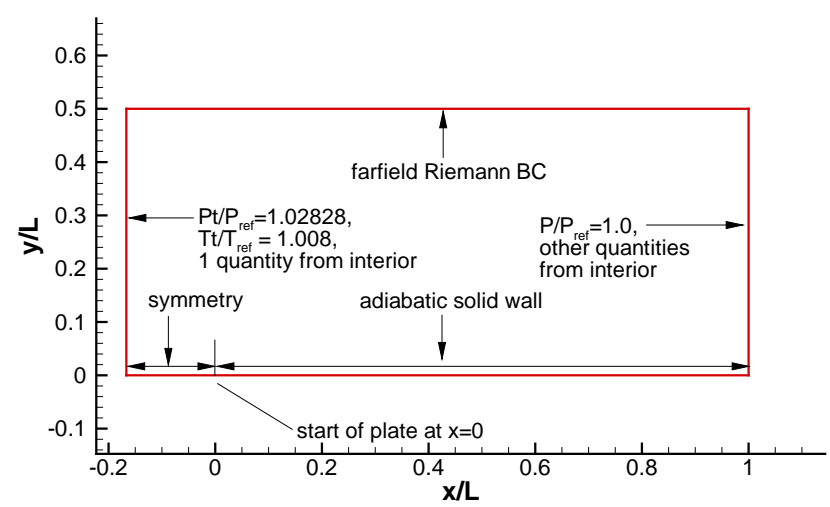

Figure 1. Flow over a flat plate at zero pressure gradient. Boundary conditions according to TMR Website. ${ }^{7}$
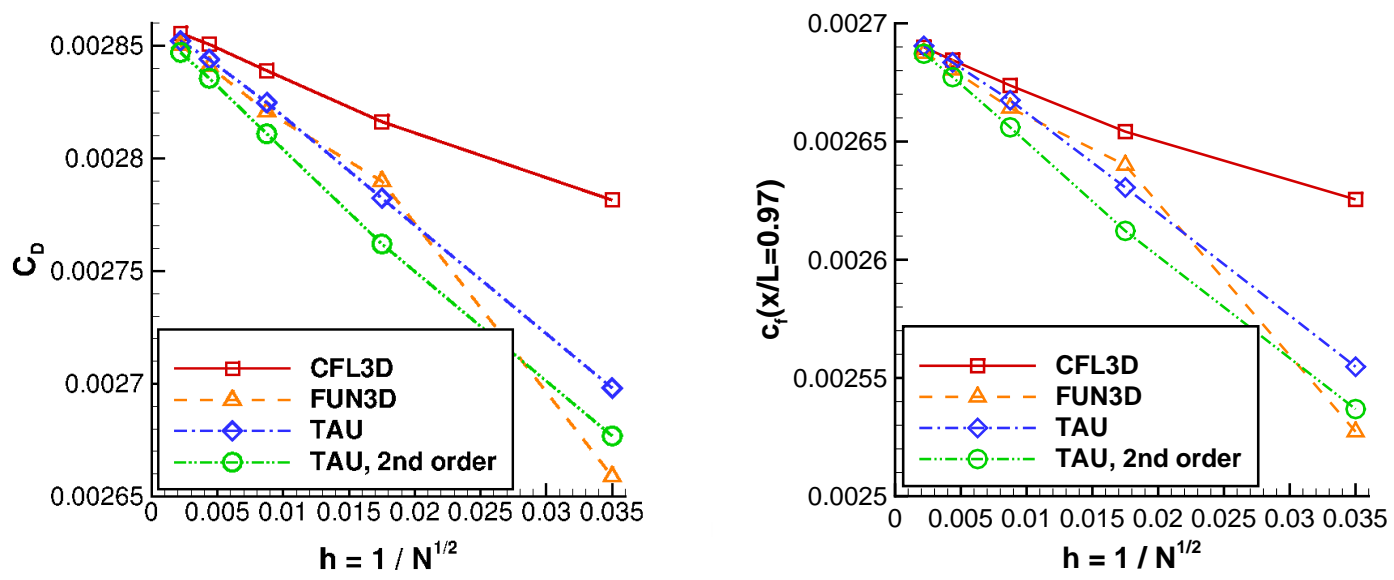

Figure 2. Flow over a flat plate at zero pressure gradient. Grid convergence of total drag coefficient (left) and local skin friction coefficient at $x / L=0.485$ (right). 2nd order refers to turbulence advection treatment.

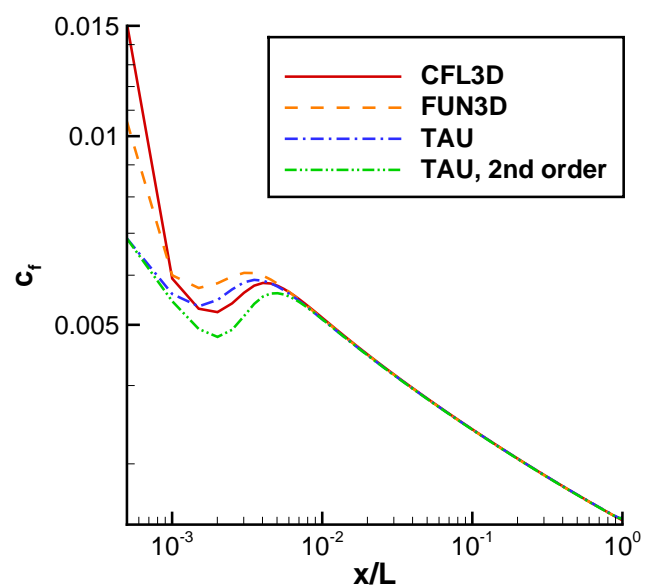

Figure 3. Flow over a flat plate at zero pressure gradient. Predicted skin friction distributions on the finest mesh with $N=545 \times 385$ grid points. 


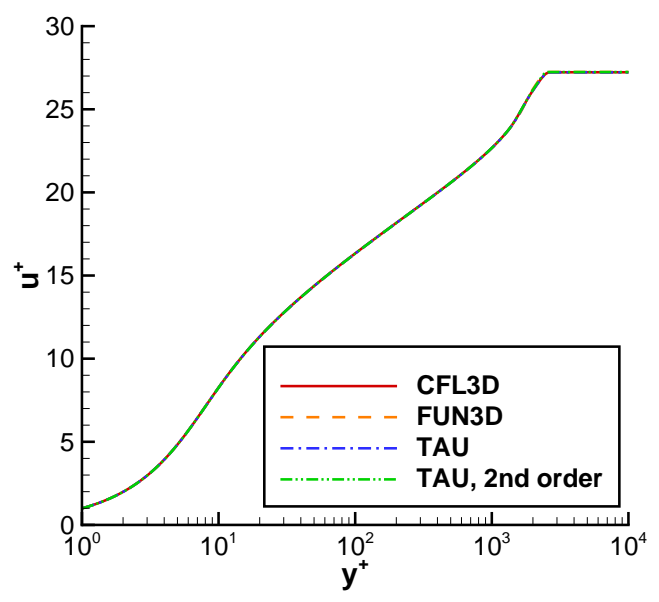

Figure 4. Flow over a flat plate at zero pressure gradient. Predicted velocity profiles in inner scaling at position $x / L=0.485$. Results for finest mesh with $N=545 \times 385$ grid points.
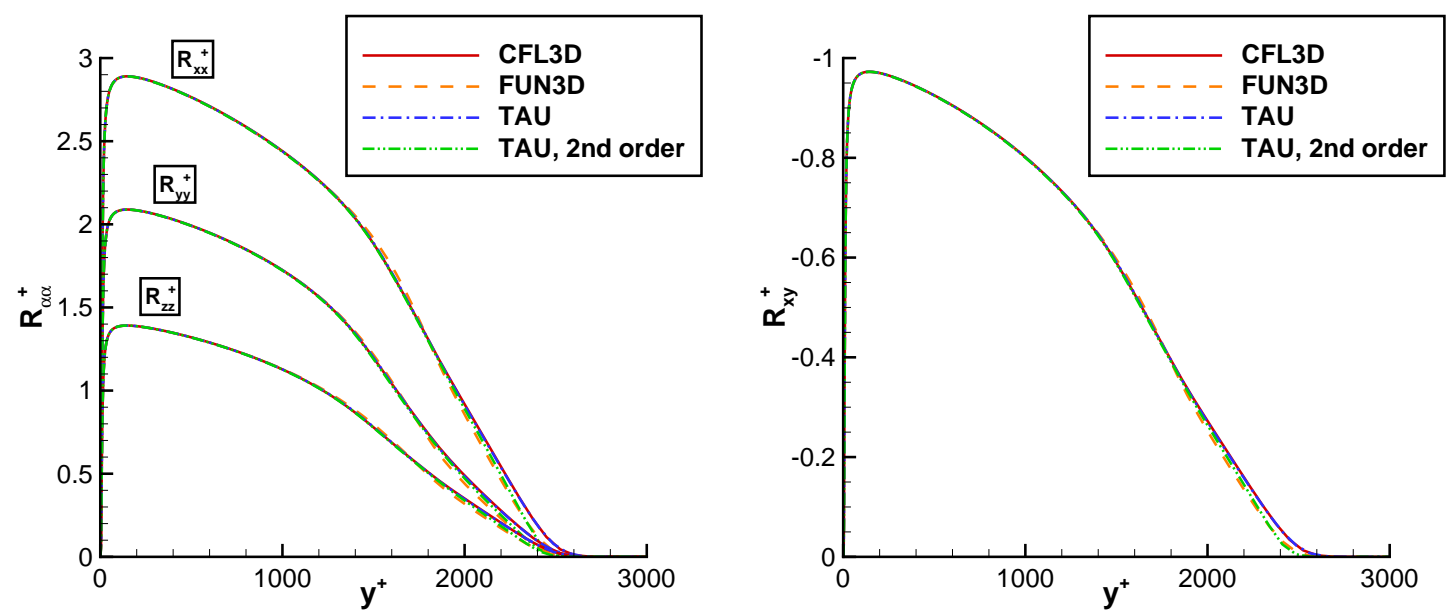

Figure 5. Flow over a flat plate at zero pressure gradient. Predicted Reynolds stress profiles in inner scaling at position $x / L=0.485$. Results for finest mesh with $N=545 \times 385$ grid points. 

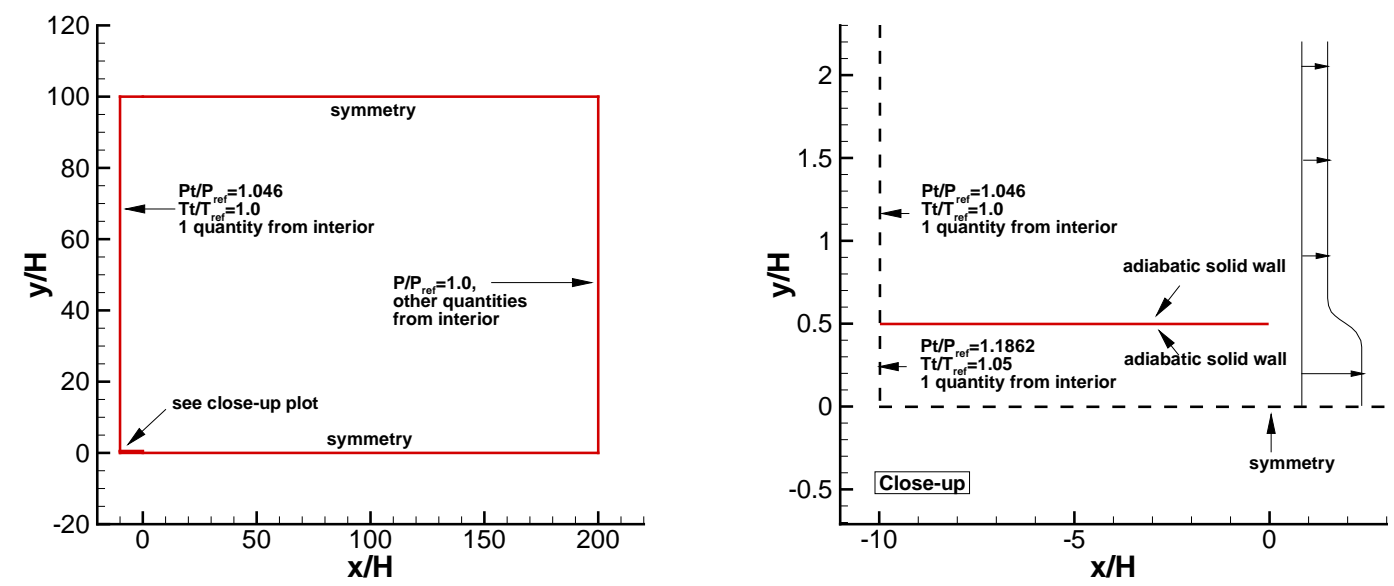

Figure 6. Plane jet developing behind an orifice. Boundary conditions according to TMR Website. ${ }^{7}$
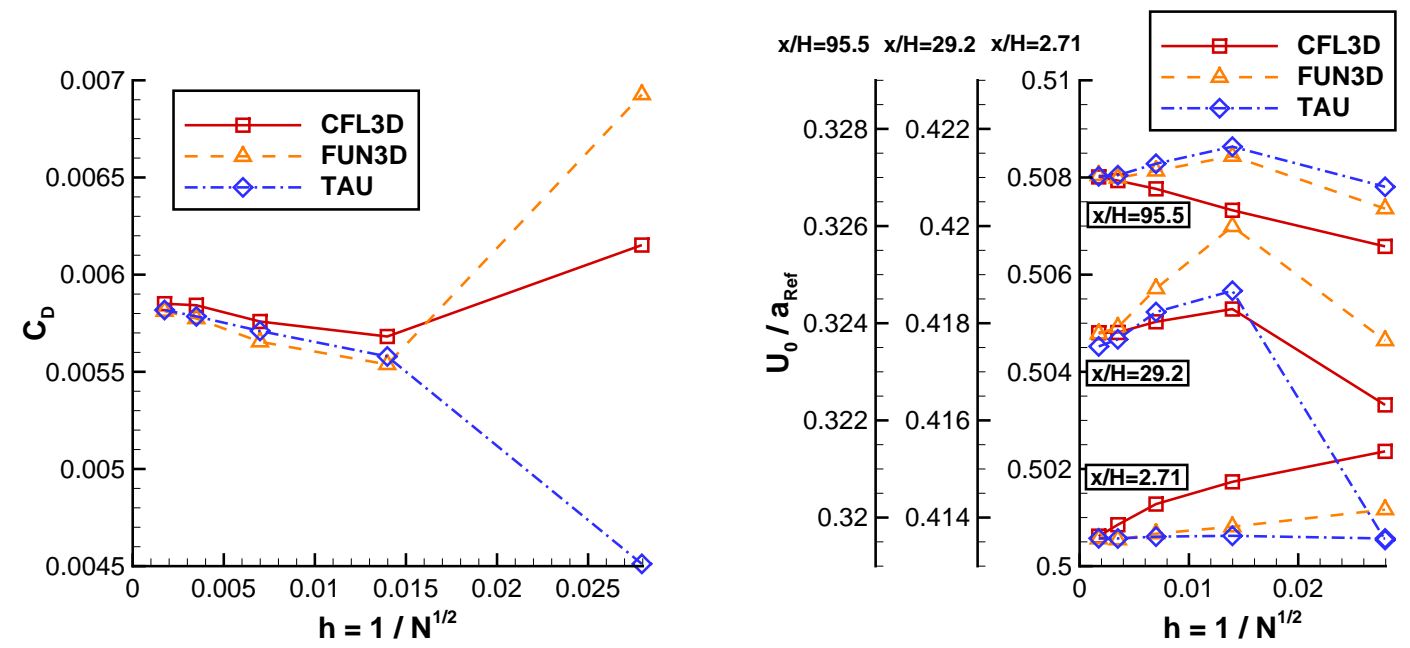

Figure 7. Plane jet developing behind an orifice. Grid convergence of total drag coefficient on the plate (left) and the non-dimensional streamwise velocity velocity $U_{0} / a_{R e f}$ on the jet centerline (right).

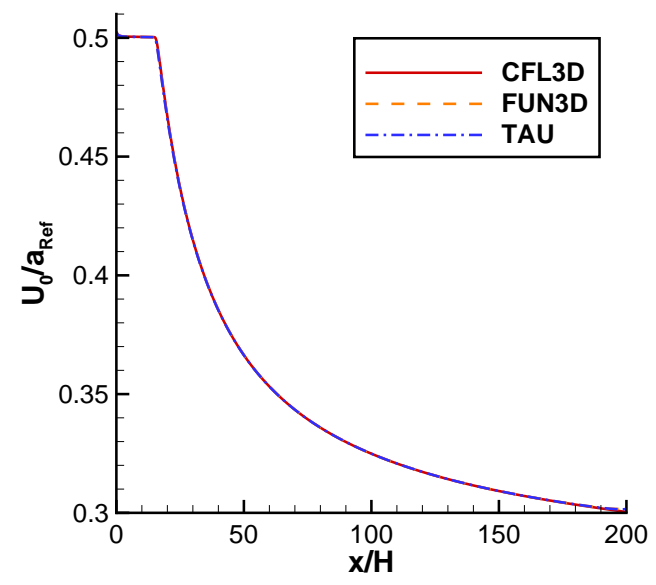

Figure 8. Plane jet developing behind an orifice. Non-dimensional streamwise velocity $U_{0} / a_{R e f}$ on the jet centerline computed on the finest mesh with $N=327,680$ points. 


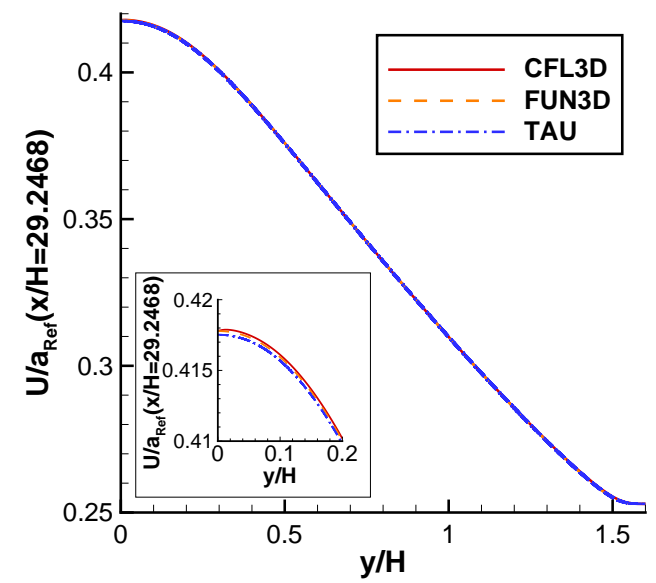

Figure 9. Plane jet developing behind an orifice. Profile of non-dimensional streamwise velocity across the jet at position $x / H=29.2468$. Results on finest mesh with $N=327,680$ points.
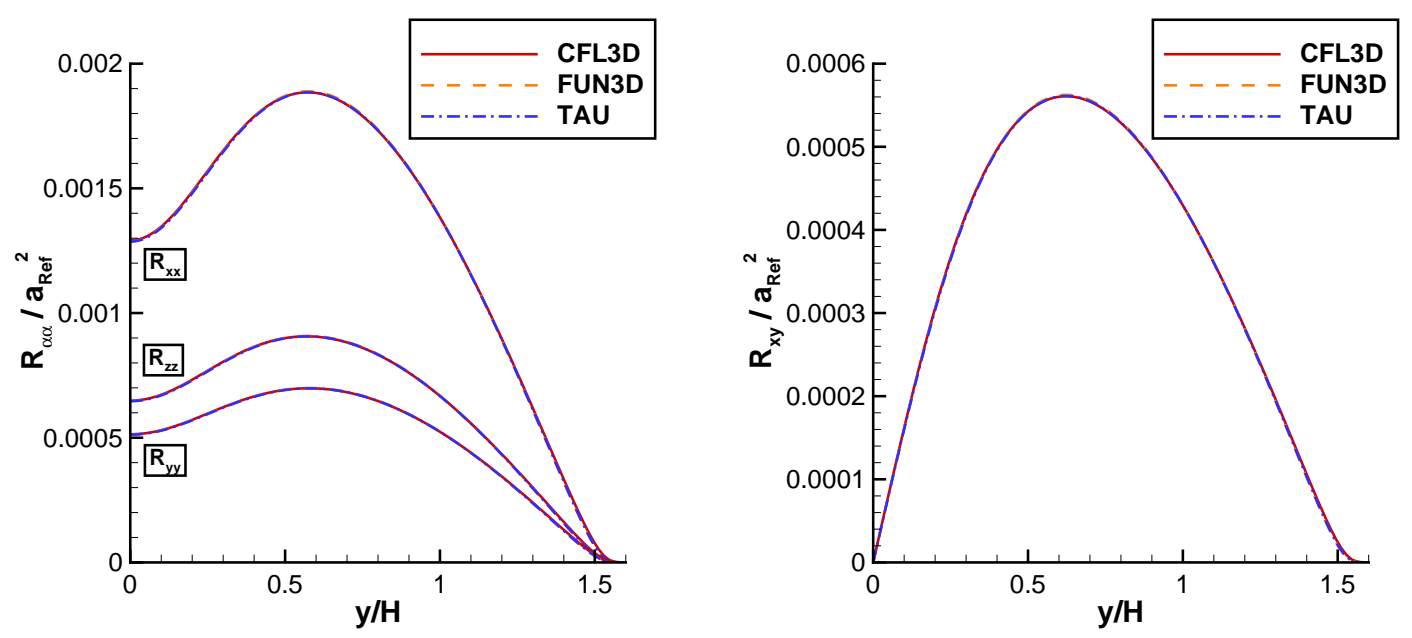

Figure 10. Plane jet developing behind an orifice. Profiles of non-dimensional Reynolds stresses across the jet at position $x / H=29.2468\left(R_{\alpha \alpha}\right.$ represents $R_{x x}, R_{y y}$, or $\left.R_{z z}\right)$. Results on finest mesh with $N=327,680$ points. 

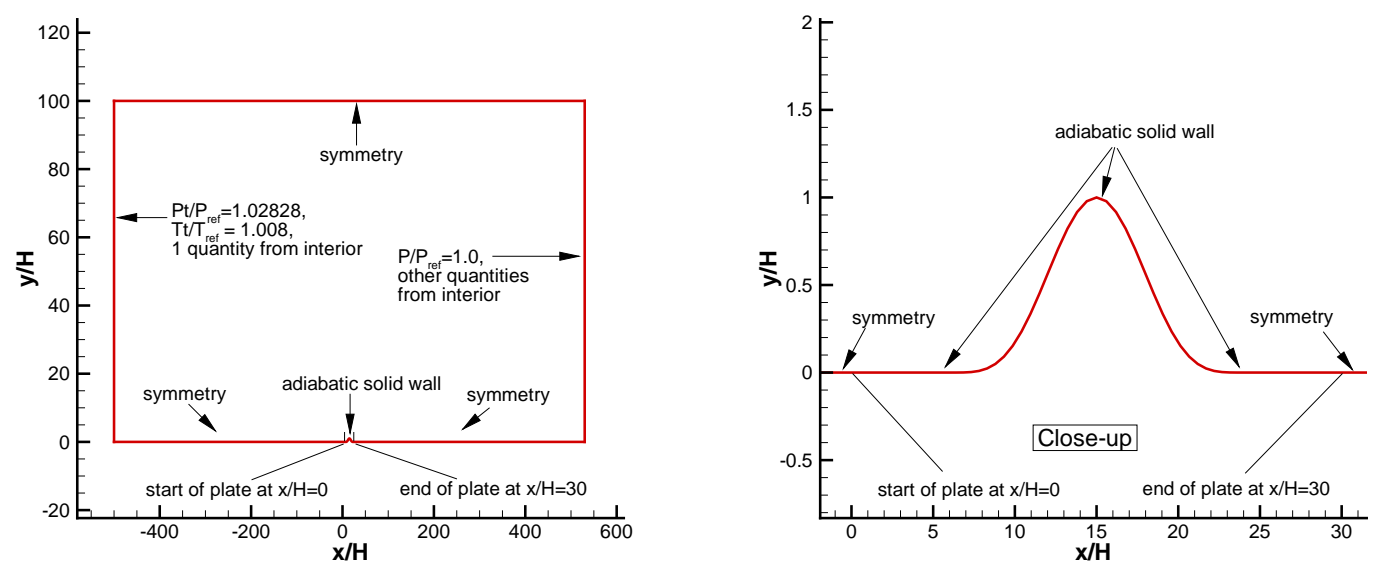

Figure 11. Two-dimensional bump in a channel. Boundary conditions according to TMR Website. ${ }^{7}$
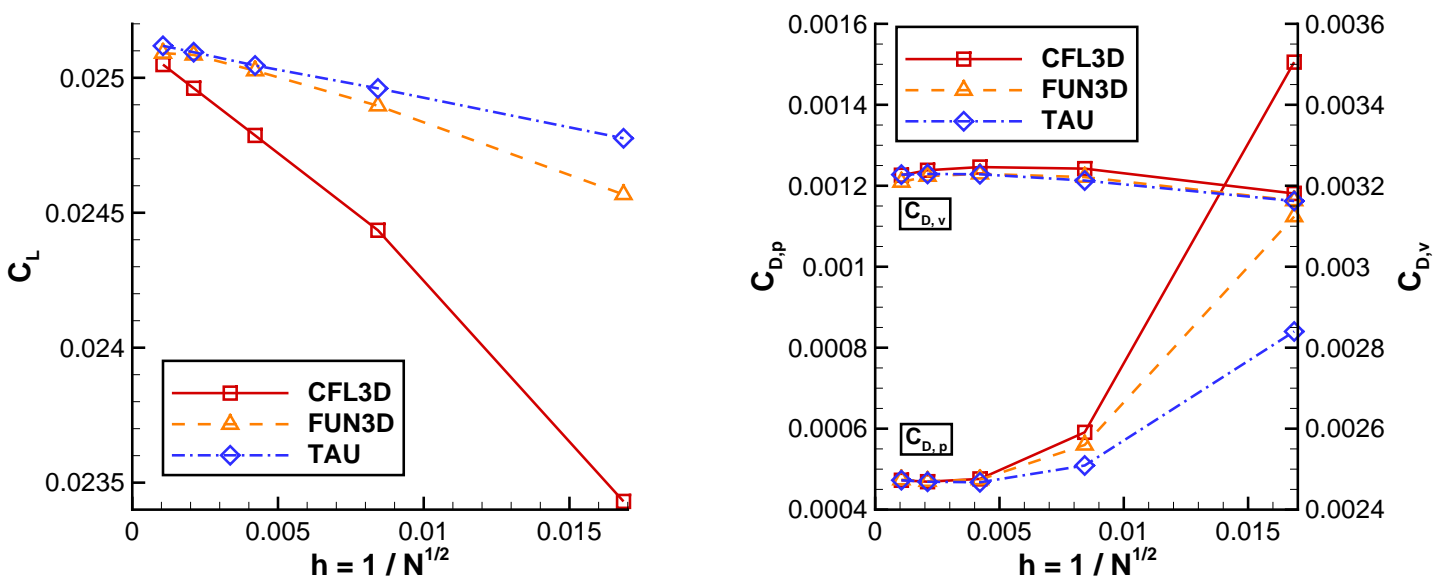

Figure 12. Two-dimensional bump in a channel. Grid convergence of lift coefficient (left) and viscous and pressure drag coefficient (right).

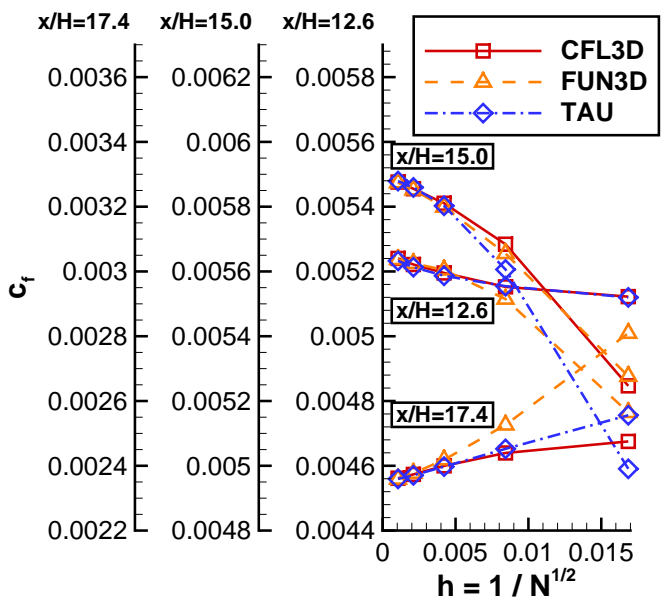

Figure 13. Two-dimensional bump in a channel. Grid convergence of local skin friction coefficient at three different stations. 

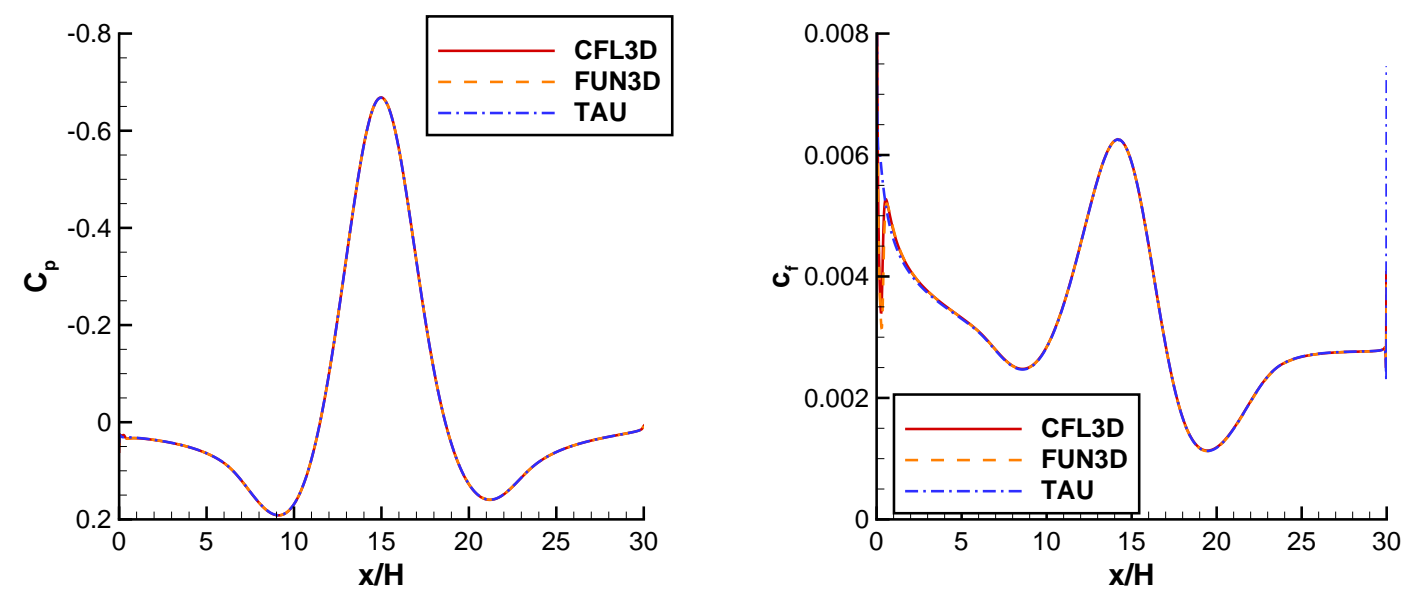

Figure 14. Two-dimensional bump in a channel. Pressure distribution (left) and skin friction distribution (right) computed on the finest mesh with $N=1,409 \times 641$ points.

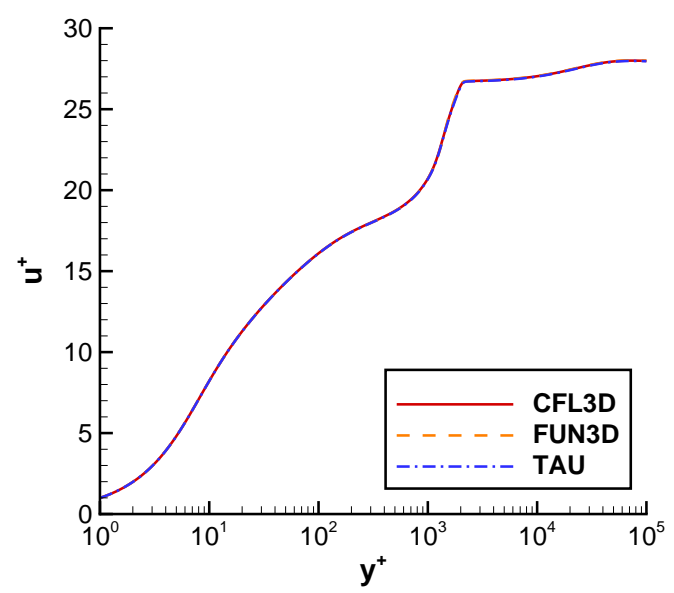

Figure 15. Two-dimensional bump in a channel. Velocity profile in inner scaling at position $x / H=24$. Results on the finest mesh with $N=1,409 \times 641$ points.
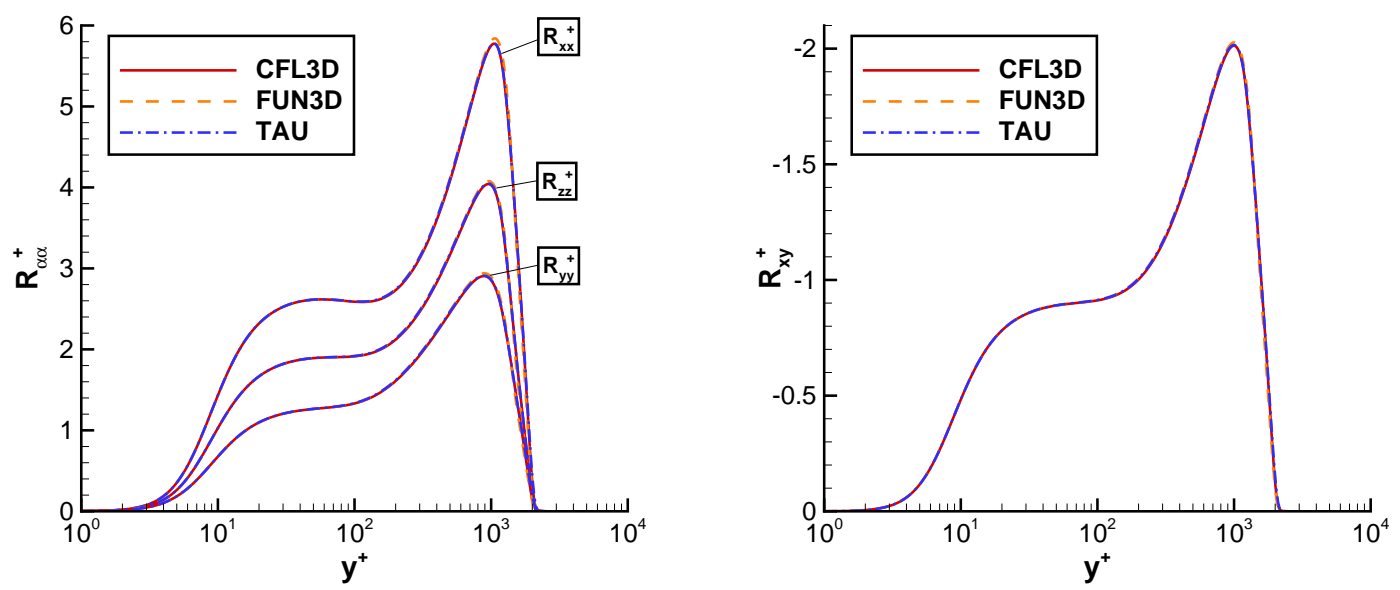

Figure 16. Two-dimensional bump in a channel. Reynolds stress profiles in inner scaling at position $x / H=24\left(R_{\alpha \alpha}^{+}\right.$ represents $R_{x x}^{+}, R_{y y}^{+}$, or $R_{z z}^{+}$). Results on the finest mesh with $N=1,409 \times 641$ points. 

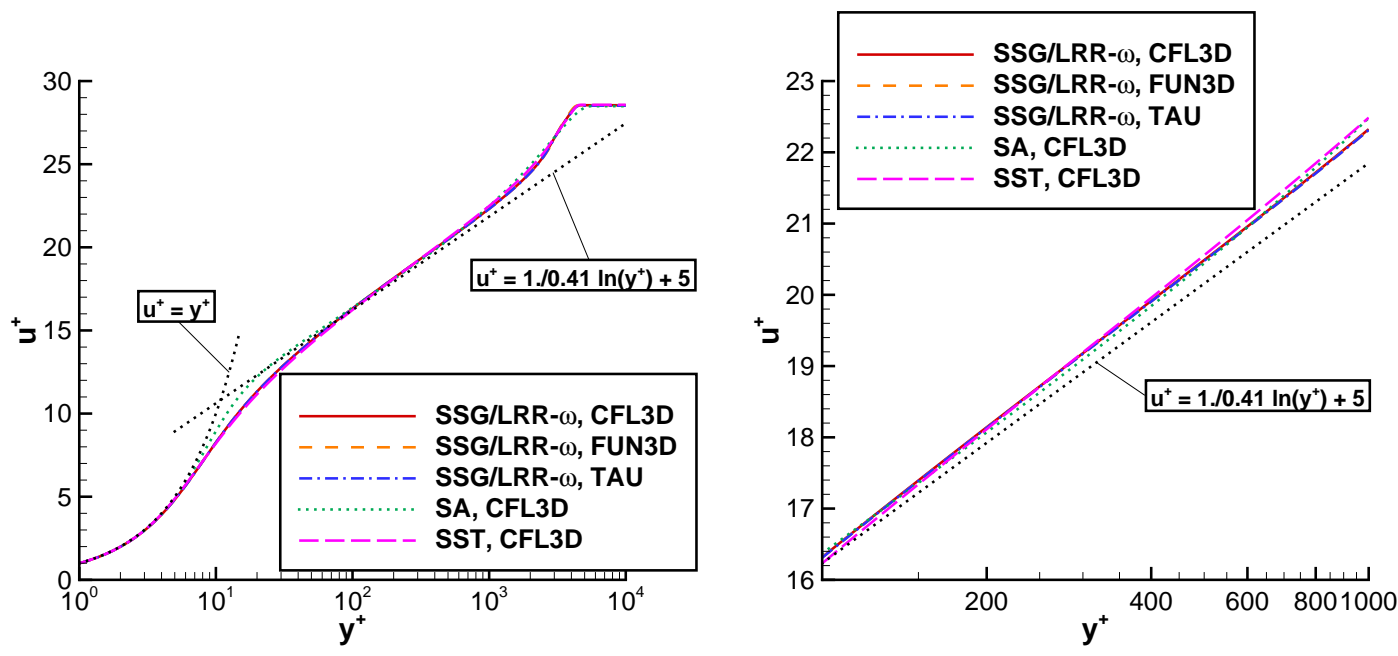

Figure 17. Flow over a flat plate at zero pressure gradient. Velocity profile in inner scaling. Comparison of numerical predictions on the finest mesh with $N=545 \times 385$ points with analytical descriptions of the viscous sublayer and the log-law.
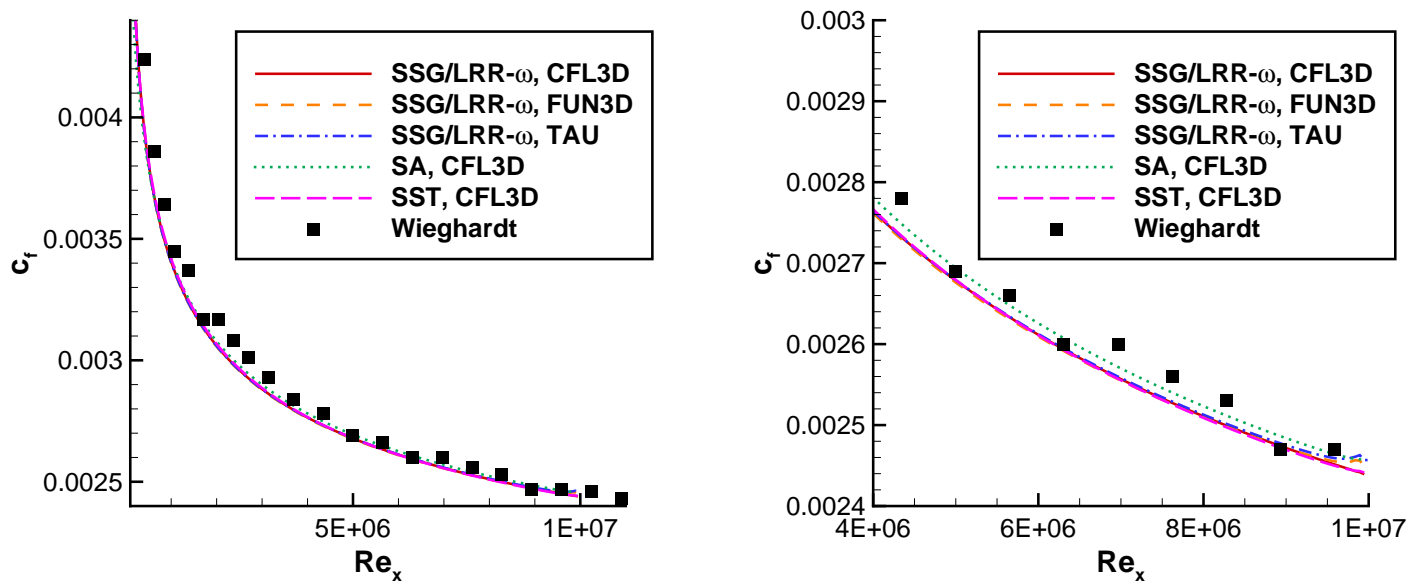

Figure 18. Flow over a flat plate at zero pressure gradient. Skin friction coefficient vs. local Reynolds number. Comparison of numerical results on the finest mesh with $N=545 \times 385$ points with experiments by Wieghardt ${ }^{18}$ as provided by Coles and Hirst. ${ }^{20}$
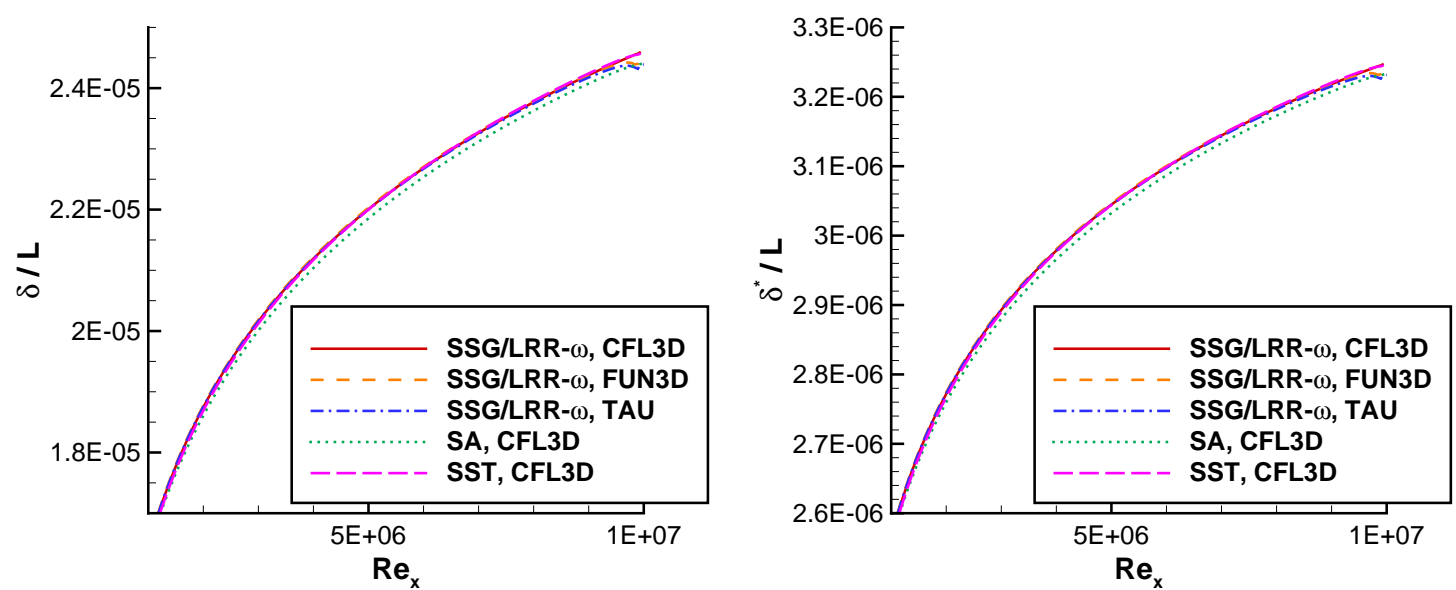

Figure 19. Flow over a flat plate at zero pressure gradient. Non-dimensional boundary layer thickness according to theoretical equation (24) (left) and displacement thickness according to theoretical equation (26) (right) for $R e_{L}=10 \cdot 10^{6}$ using numerically predicted $c_{f}$-distributions. 


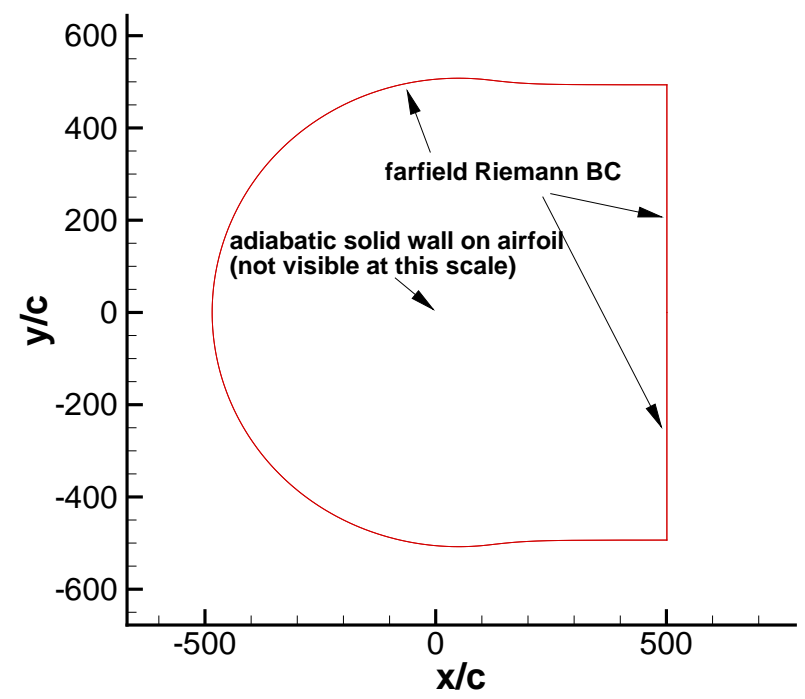

Figure 20. Subsonic flow around NACA 0012 airfoil. Boundary conditions according to TMR Website. ${ }^{7}$
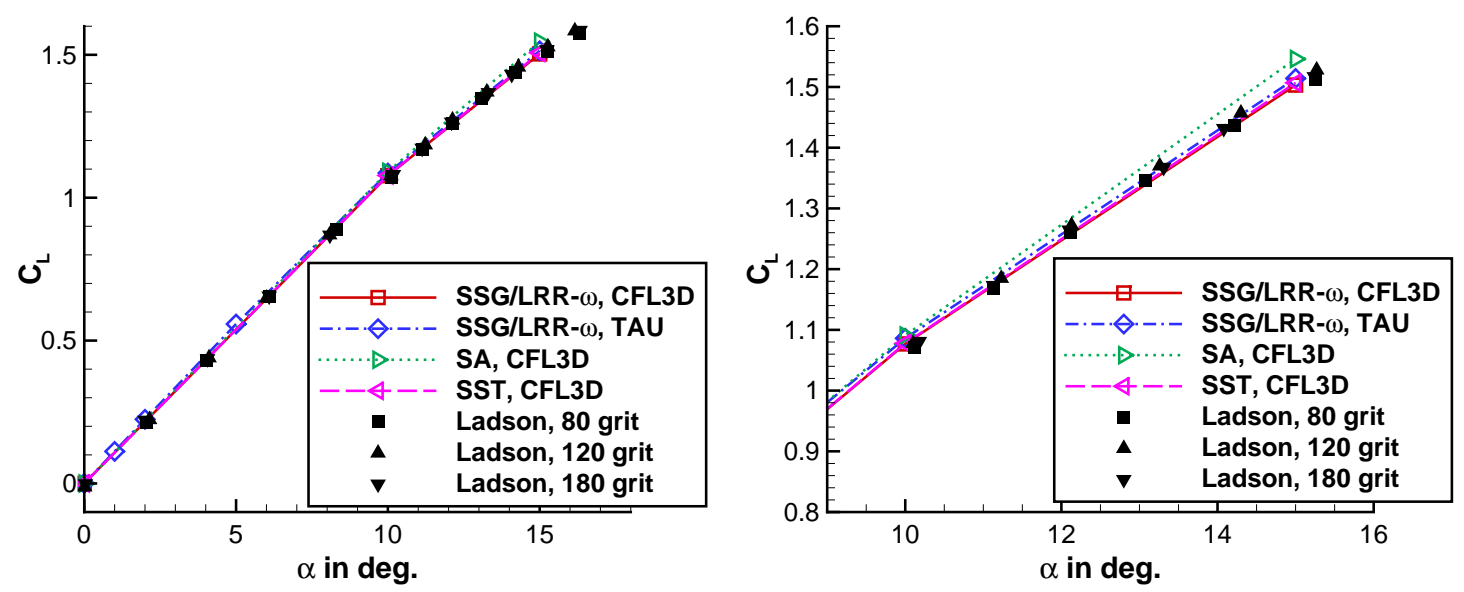

Figure 21. Subsonic flow around NACA 0012 airfoil. Lift coefficient versus incidence. Comparison of numerical results on the second finest grid with $N=897 \times 257$ points with experiments by Ladson. ${ }^{22}$

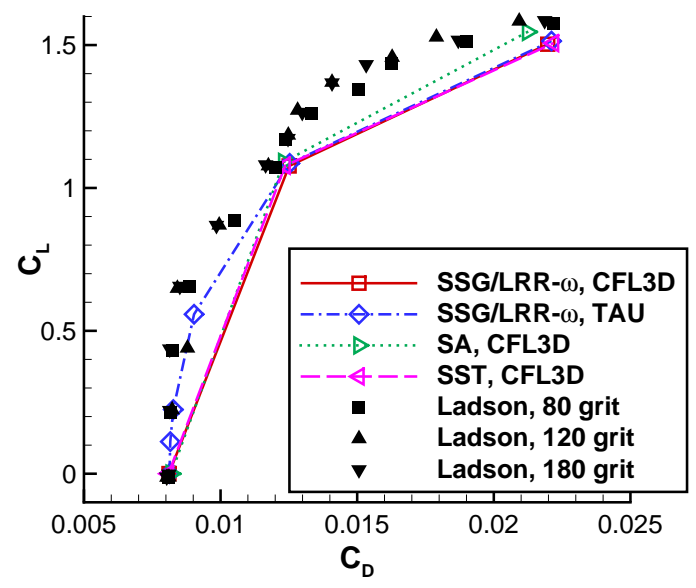

Figure 22. Subsonic flow around NACA 0012 airfoil. Force polar. Comparison of numerical results on the second finest grid with $N=897 \times 257$ points with experiments by Ladson. ${ }^{22}$ 

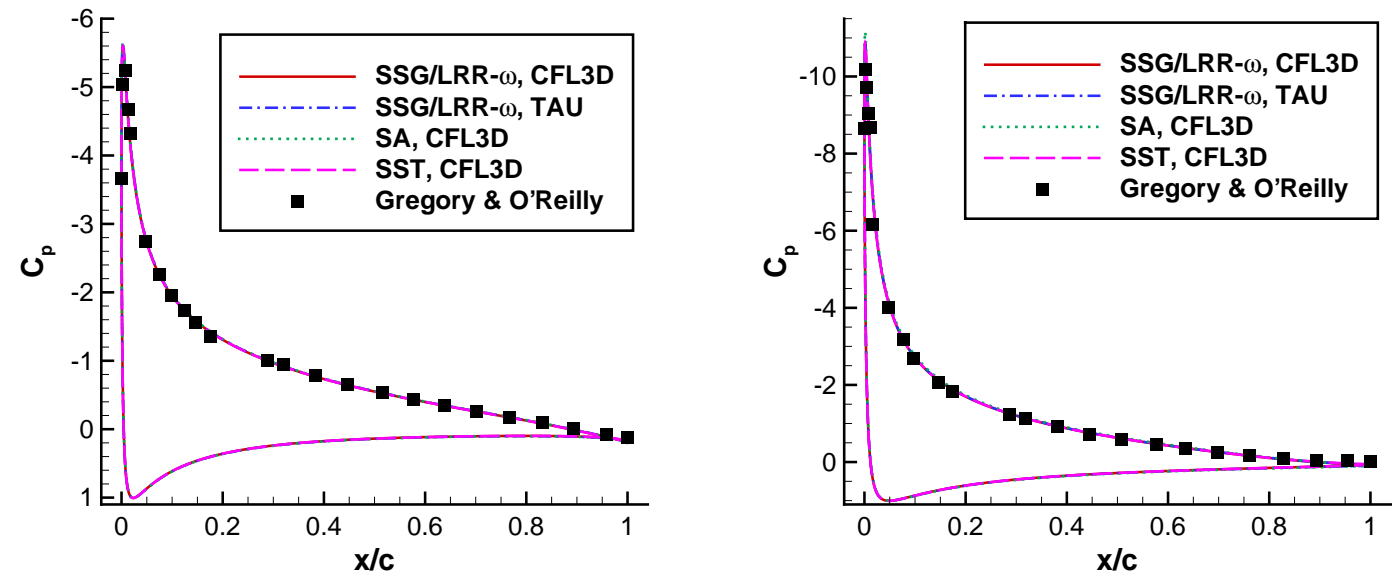

Figure 23. Subsonic flow around NACA 0012 airfoil. Pressure distribution at $\alpha=10^{\circ}$ and $\alpha=15^{\circ}$. Comparison of numerical results on the second finest grid with $N=897 \times 257$ points with experiments by Gregory and O'Reilly. ${ }^{23}$
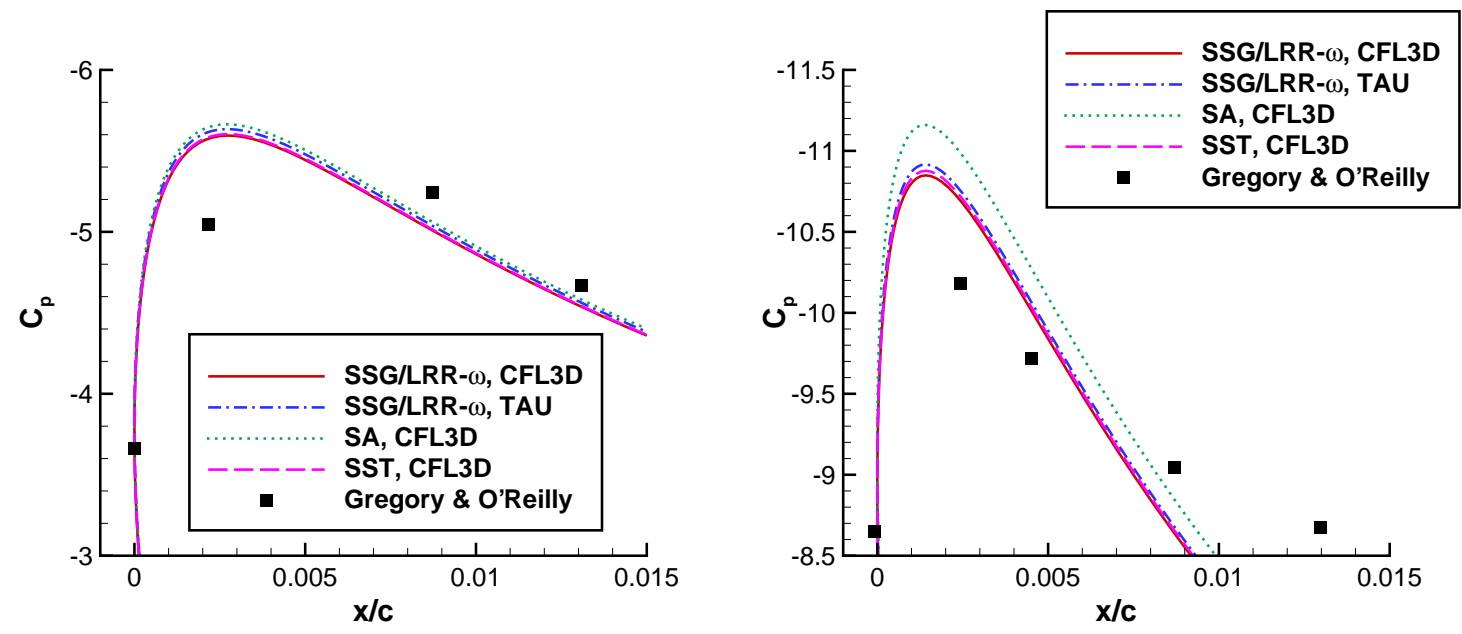

Figure 24. Subsonic flow around NACA 0012 airfoil. Detail of the pressure distribution at $\alpha=10^{\circ}$ and $\alpha=15^{\circ}$ near the suction peak. Comparison of numerical results on the second finest grid with $N=897 \times 257$ points with experiments by Gregory and O'Reilly. ${ }^{23}$ 


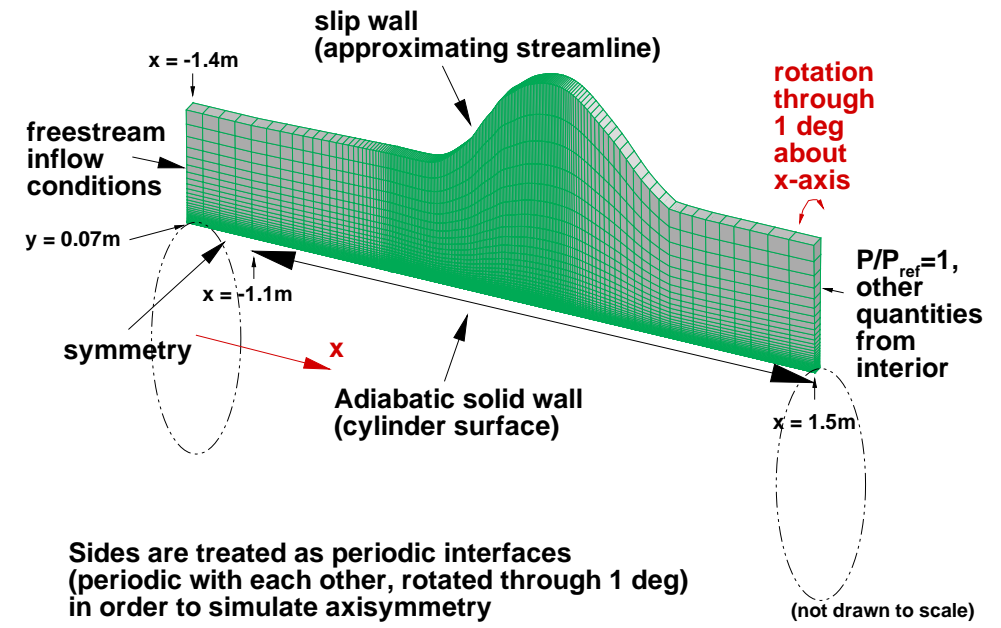

Figure 25. Axisymmetric separated boundary layer. Grid structure and boundary conditions according to TMR Website. ${ }^{7}$ Dash-dot ellipses represent the cylinder.
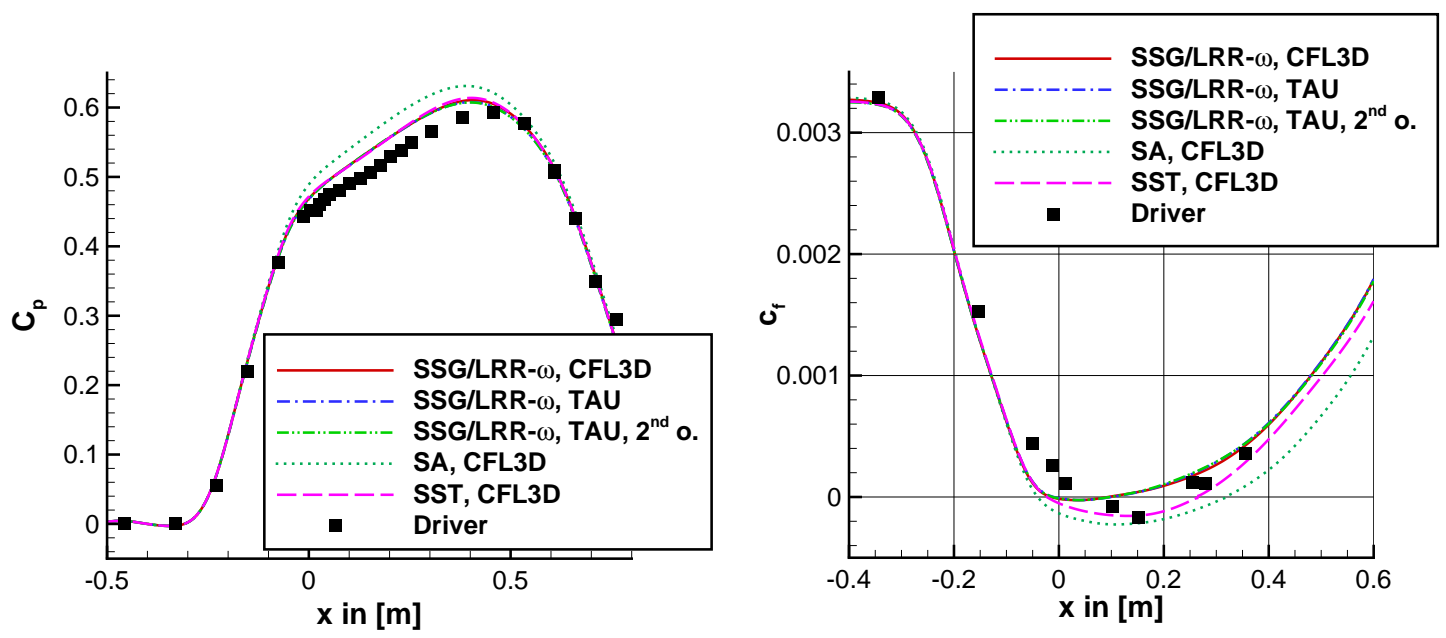

Figure 26. Axisymmetric separated boundary layer. Pressure distribution (left) and skin friction distribution (right). Comparison of numerical results on the second finest mesh with $N=713 \times 193$ points with experiments by Driver. ${ }^{24}$
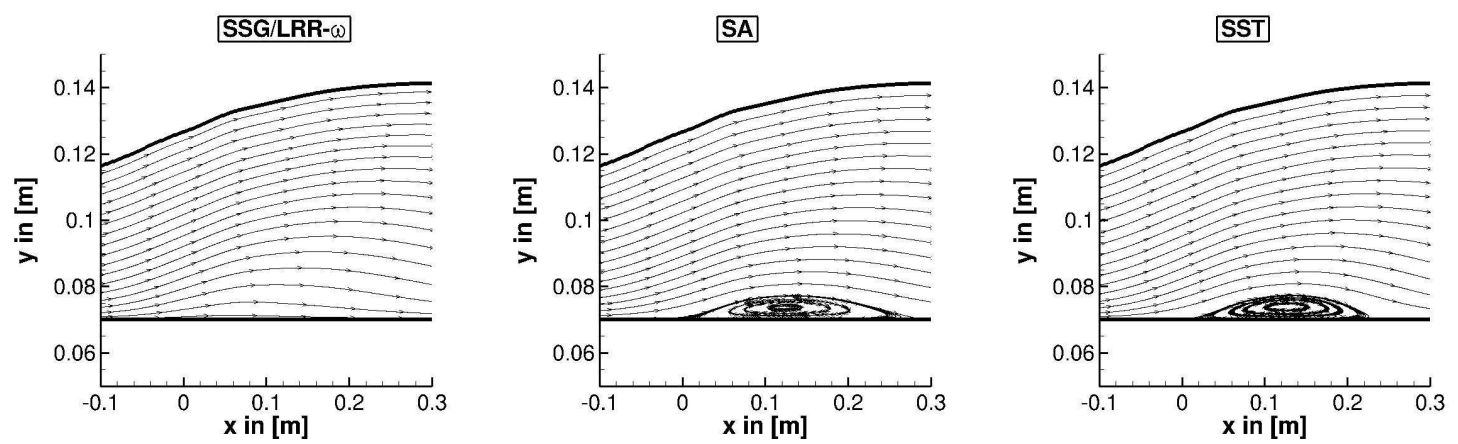

Figure 27. Axisymmetric separated boundary layer. Streamlines in the region of flow separation predicted with CFL3D using the SSG/LRR- $\omega$ model (left), the SA model (center) and the SST model (right). 

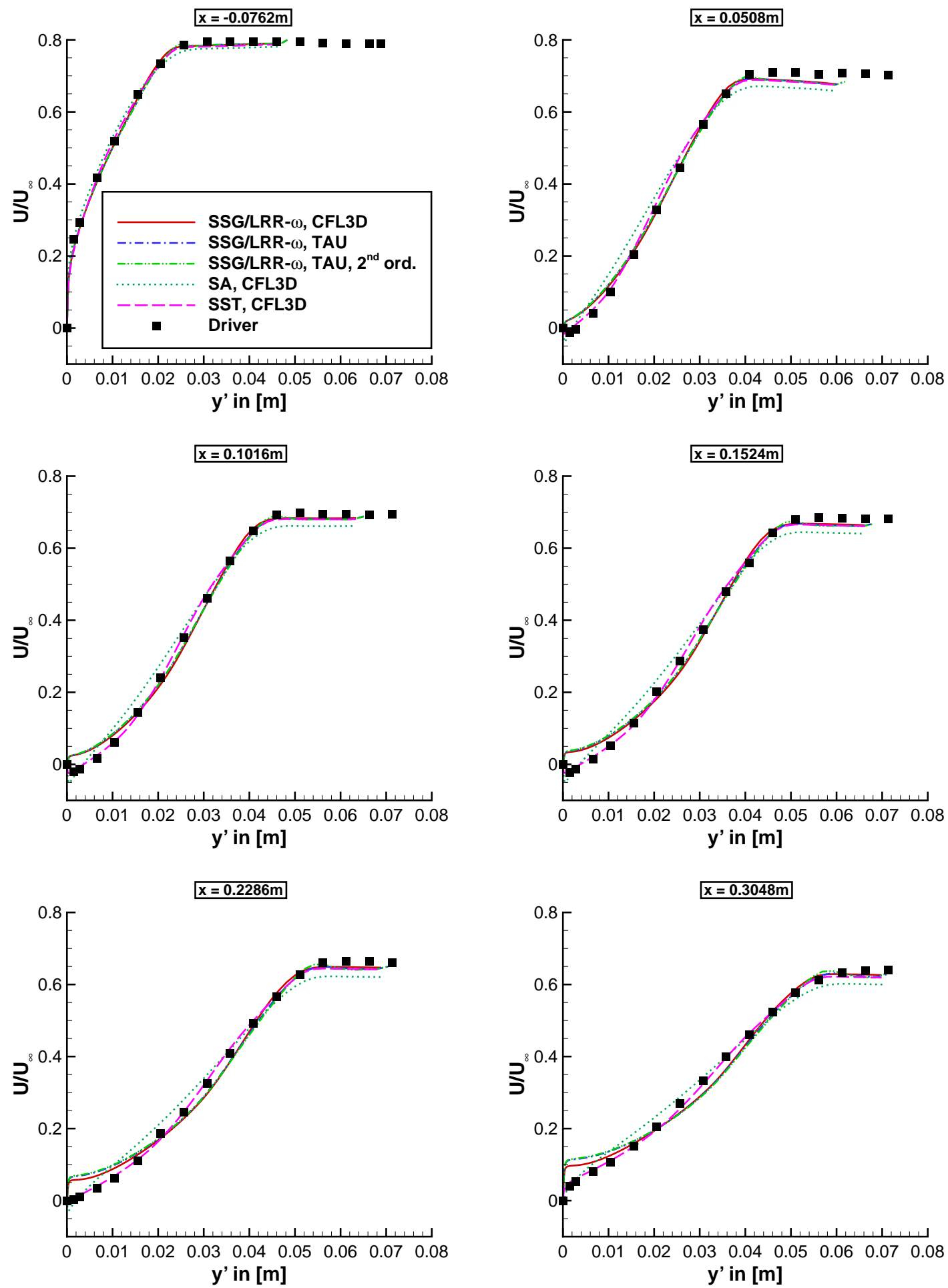

Figure 28. Axisymmetric separated boundary layer. Non-dimensional velocity profiles at different streamwise positions. $y^{\prime}$ is the wall normal coordinate originating from the surface. Comparison of numerical results on the second finest mesh with $N=713 \times 193$ points with experiments. ${ }^{24}$ 

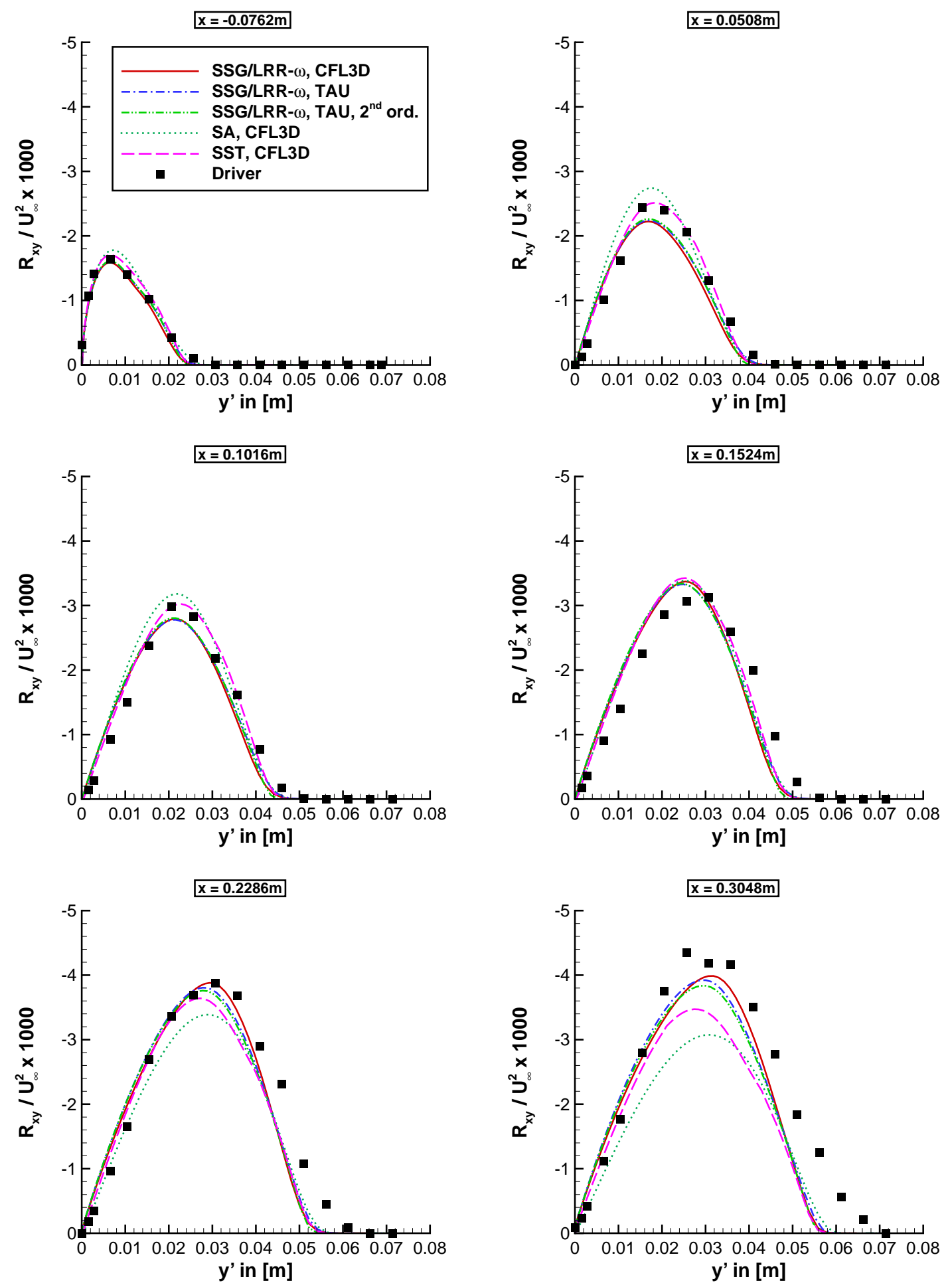

Figure 29. Axisymmetric separated boundary layer. Profiles of non-dimensional Reynolds shear stress at different streamwise positions. $y^{\prime}$ is the distance from the surface. Reynolds stresses multiplied by a factor of 1000 . Comparison of numerical results on the second finest mesh with $N=713 \times 193$ points with experiments. ${ }^{24}$ 


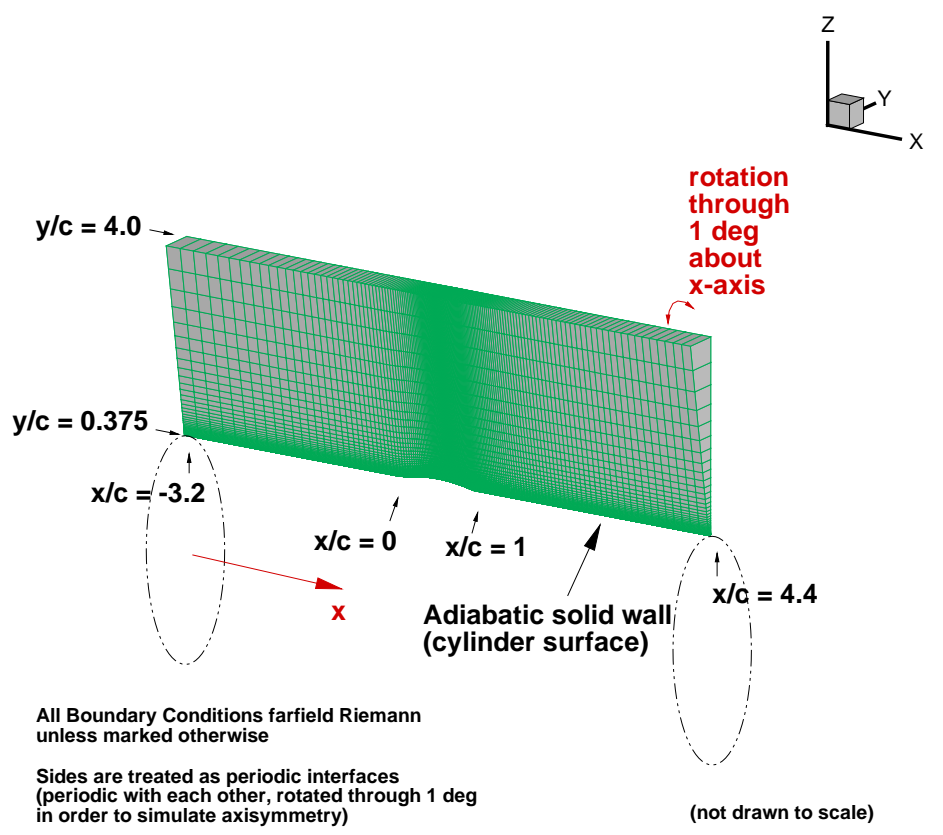

Figure 30. Transonic flow over an axisymmetric bump. Grid structure and boundary conditions according to TMR Website. ${ }^{7}$ Dash-dot ellipses represent the cylinder.
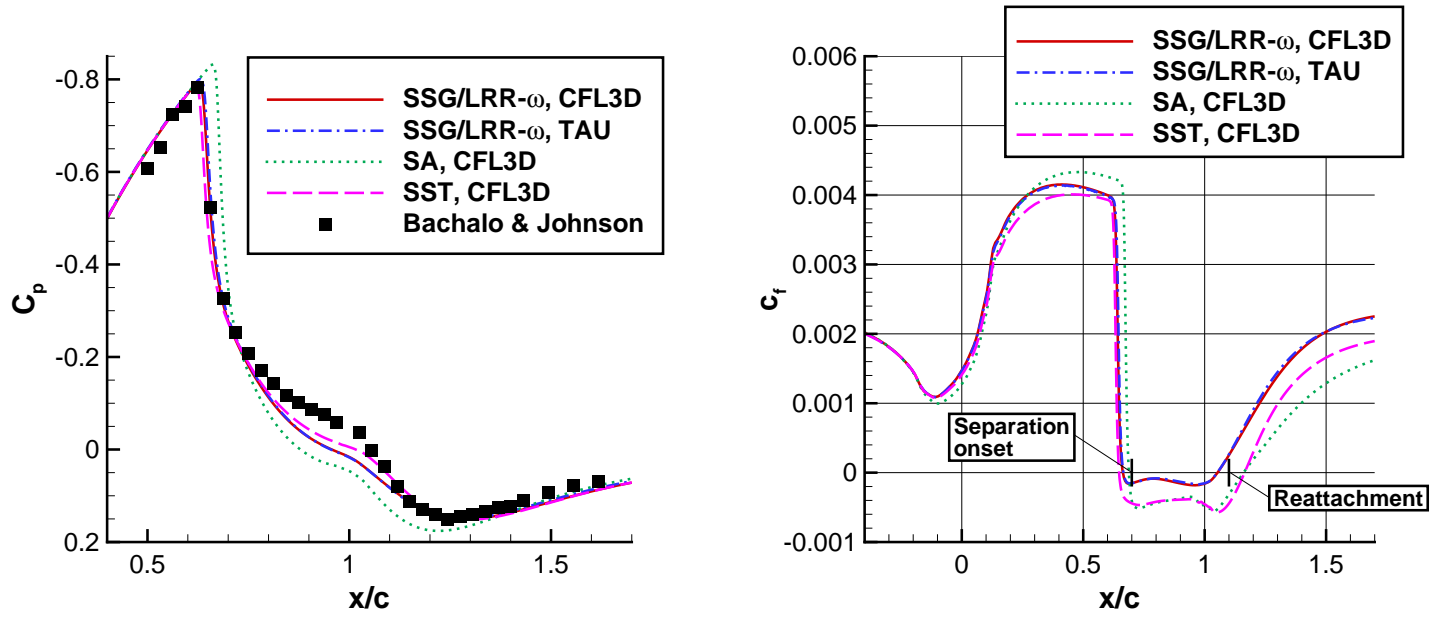

Figure 31. Transonic flow over an axisymmetric bump. Pressure distribution (left) and skin friction distribution (right). Comparison of numerical results on the second finest mesh with $N=721 \times 321$ points with $C_{p}$ data from experiments of Bachalo and Johnson. ${ }^{25}$ Experimental separation and reattachment indicated in $c_{f}$ plot by small vertical lines.
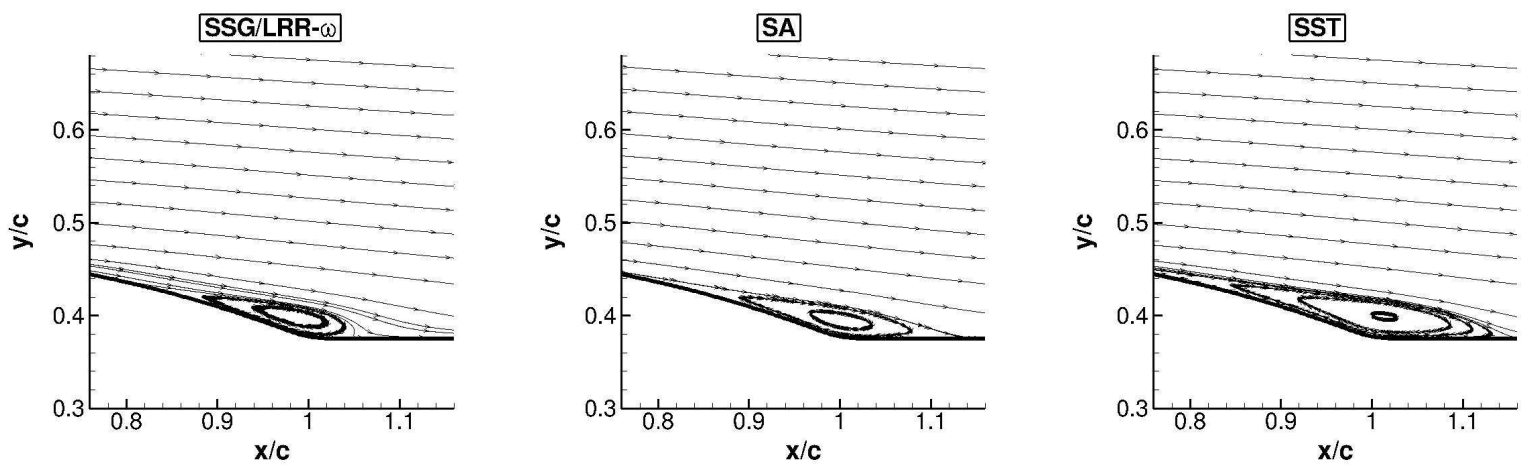

Figure 32. Transonic flow over an axisymmetric bump. Streamlines in the region of flow separation predicted with CFL3D using the SSG/LRR- $\omega$ model (left), the SA model (center) and the SST model (right). 

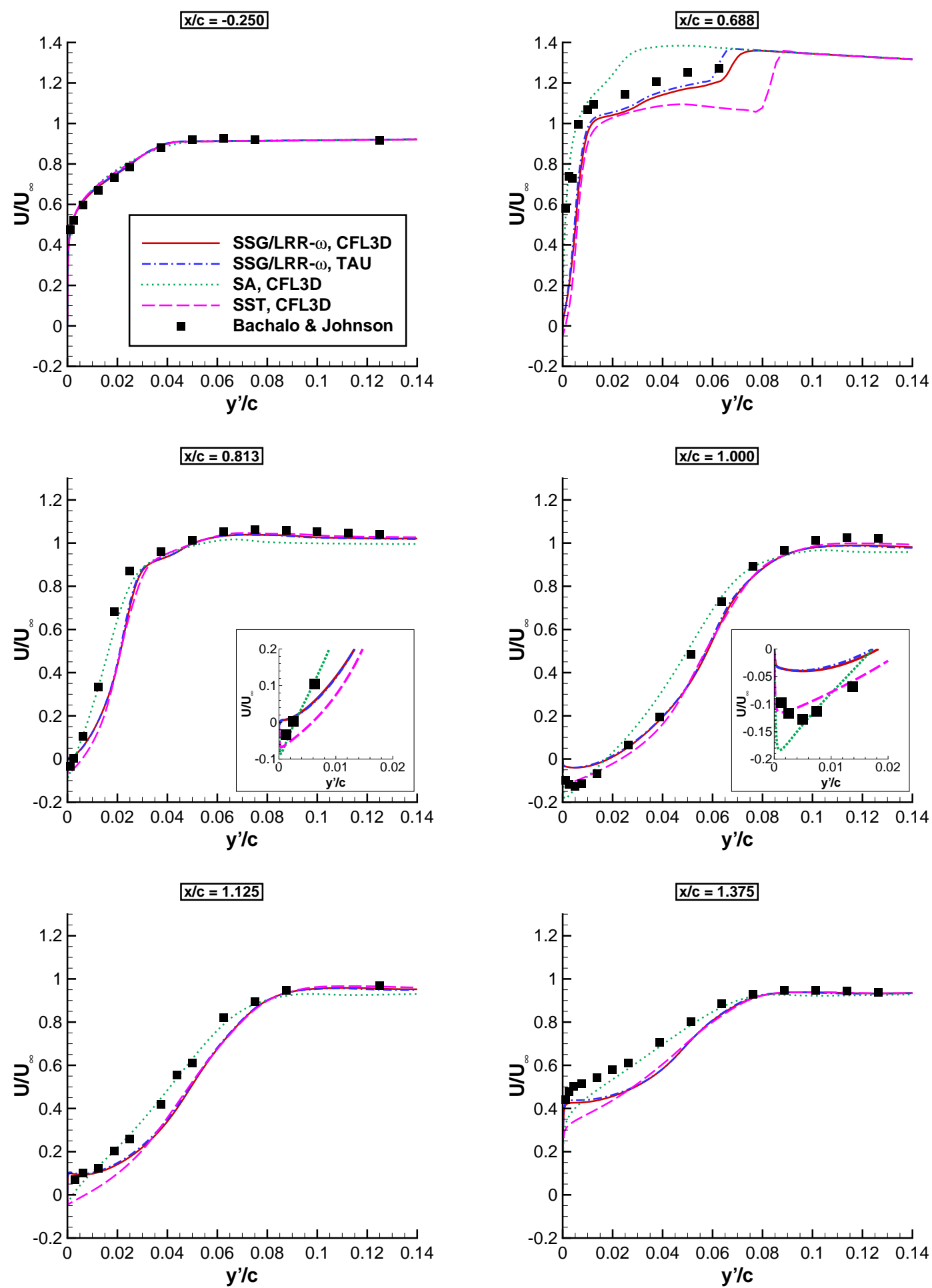

Figure 33. Transonic flow over an axisymmetric bump. Non-dimensional velocity profiles at different streamwise positions. $y^{\prime}$ is the coordinate normal to the streamwise direction, originating from the surface. Comparison of numerical results on the second finest mesh with $N=721 \times 321$ points with experiments by Bachalo and Johnson. ${ }^{25}$ 

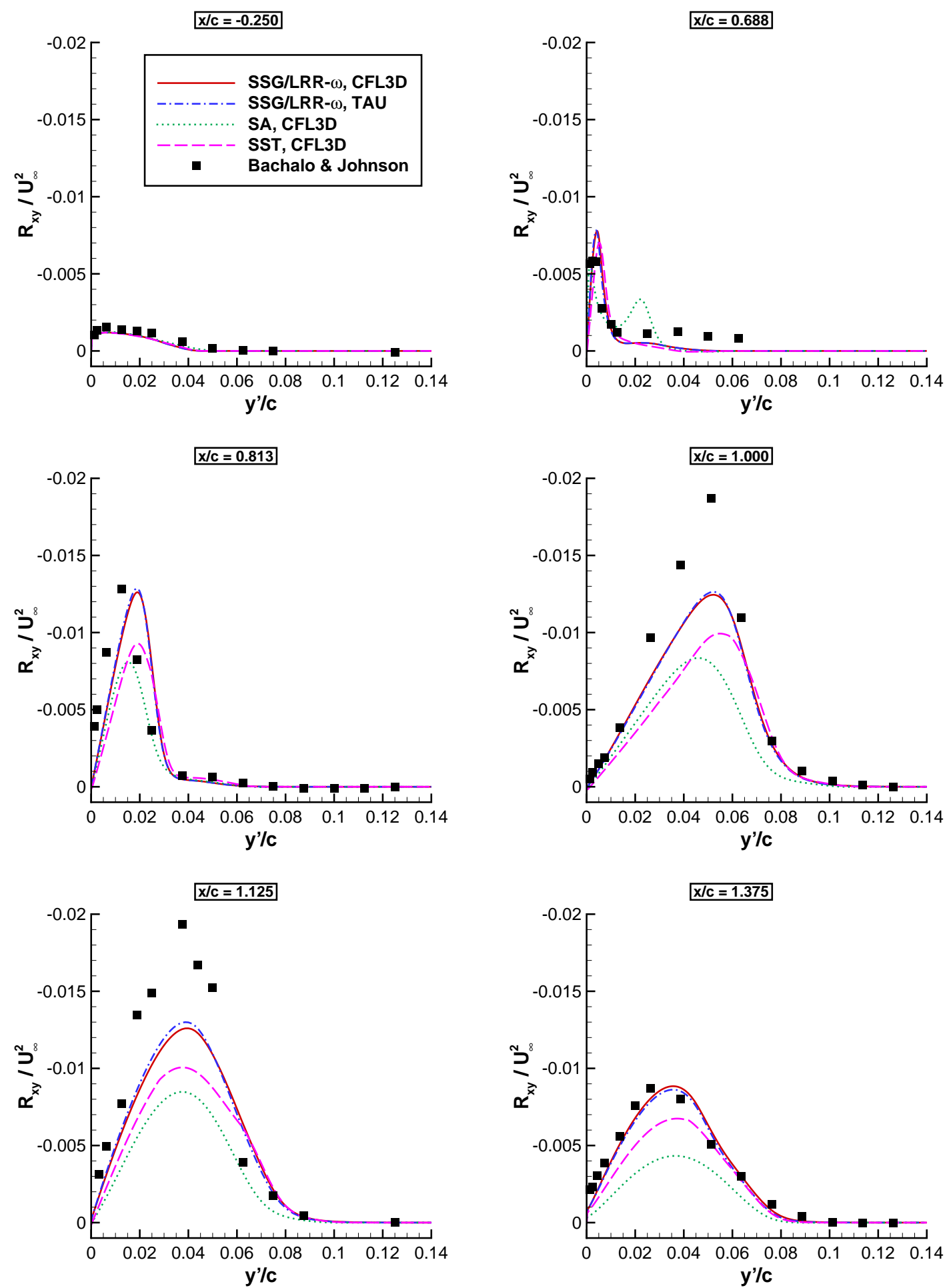

Figure 34. Transonic flow over an axisymmetric bump. Non-dimensional Reynolds shear stress profiles at different streamwise positions. $y^{\prime}$ is the coordinate normal to the streamwise direction, originating from the surface. Comparison of numerical results on the second finest mesh with $N=721 \times 321$ points with experiments by Bachalo and Johnson. ${ }^{25}$ 


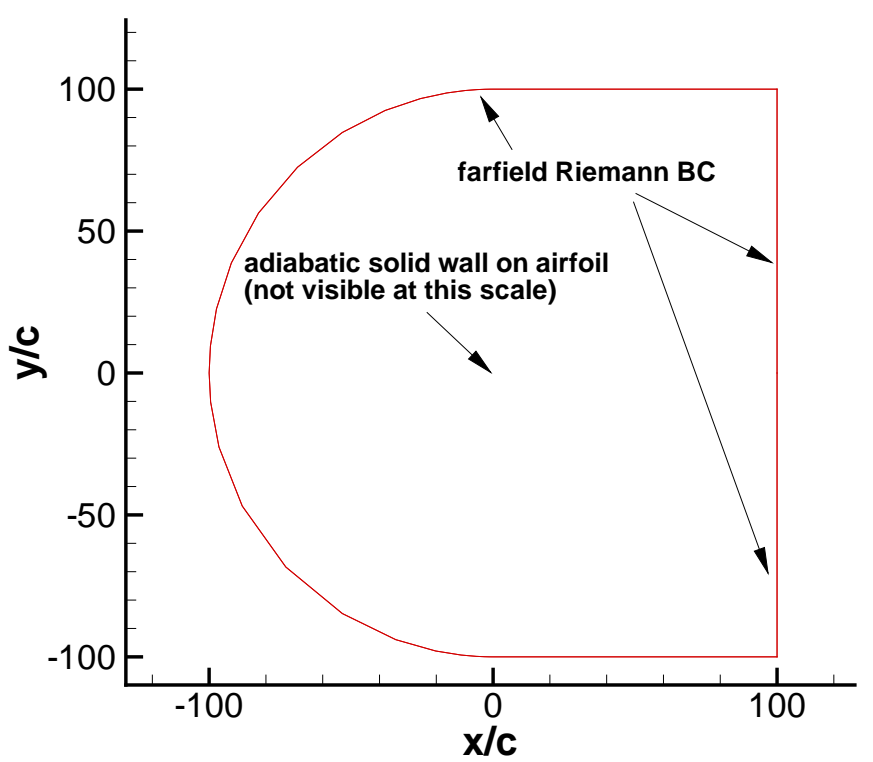

Figure 35. Subsonic flow around NACA 4412 airfoil. Boundary conditions and coordinate system, according to TMR Website. $^{7}$
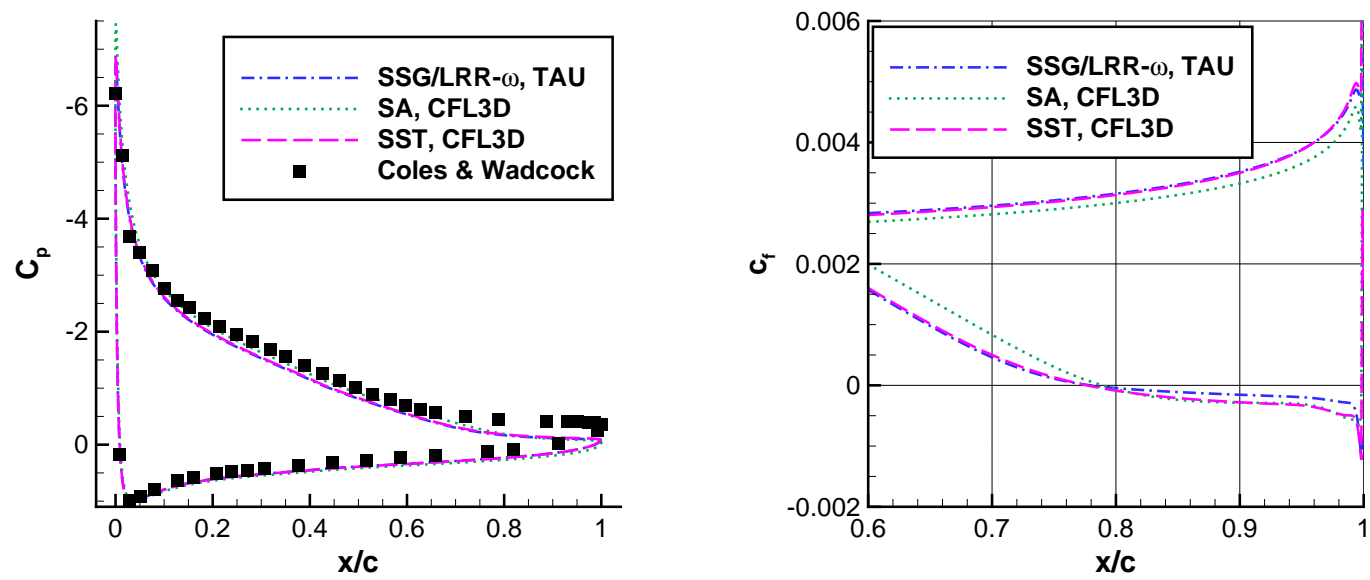

Figure 36. Subsonic flow around NACA 4412 airfoil. Pressure distribution (left) and skin friction distribution near trailing edge (right). Comparison of numerical results on the second finest mesh with $N=897 \times 257$ points with $C_{p}$ data from experiments of Coles and Wadcock. ${ }^{27,28}$
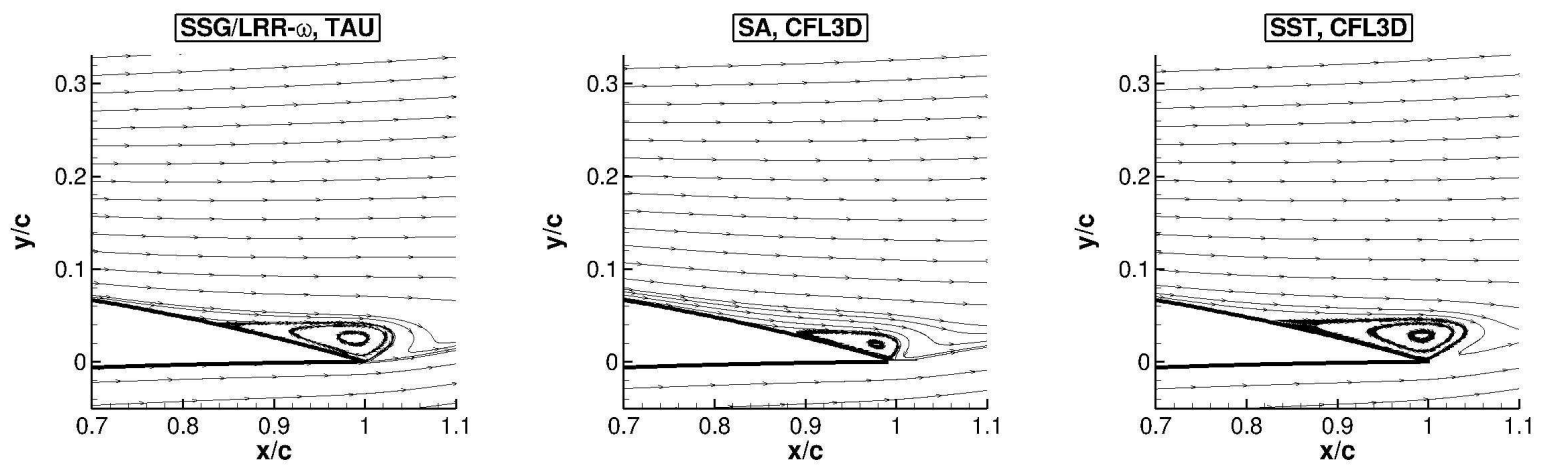

Figure 37. Subsonic flow around NACA 4412 airfoil. Streamlines in the region of flow separation predicted with TAU using the SSG/LRR- $\omega$ model (left) and with CFL3D, using the SA model (center) and the SST model (right). 

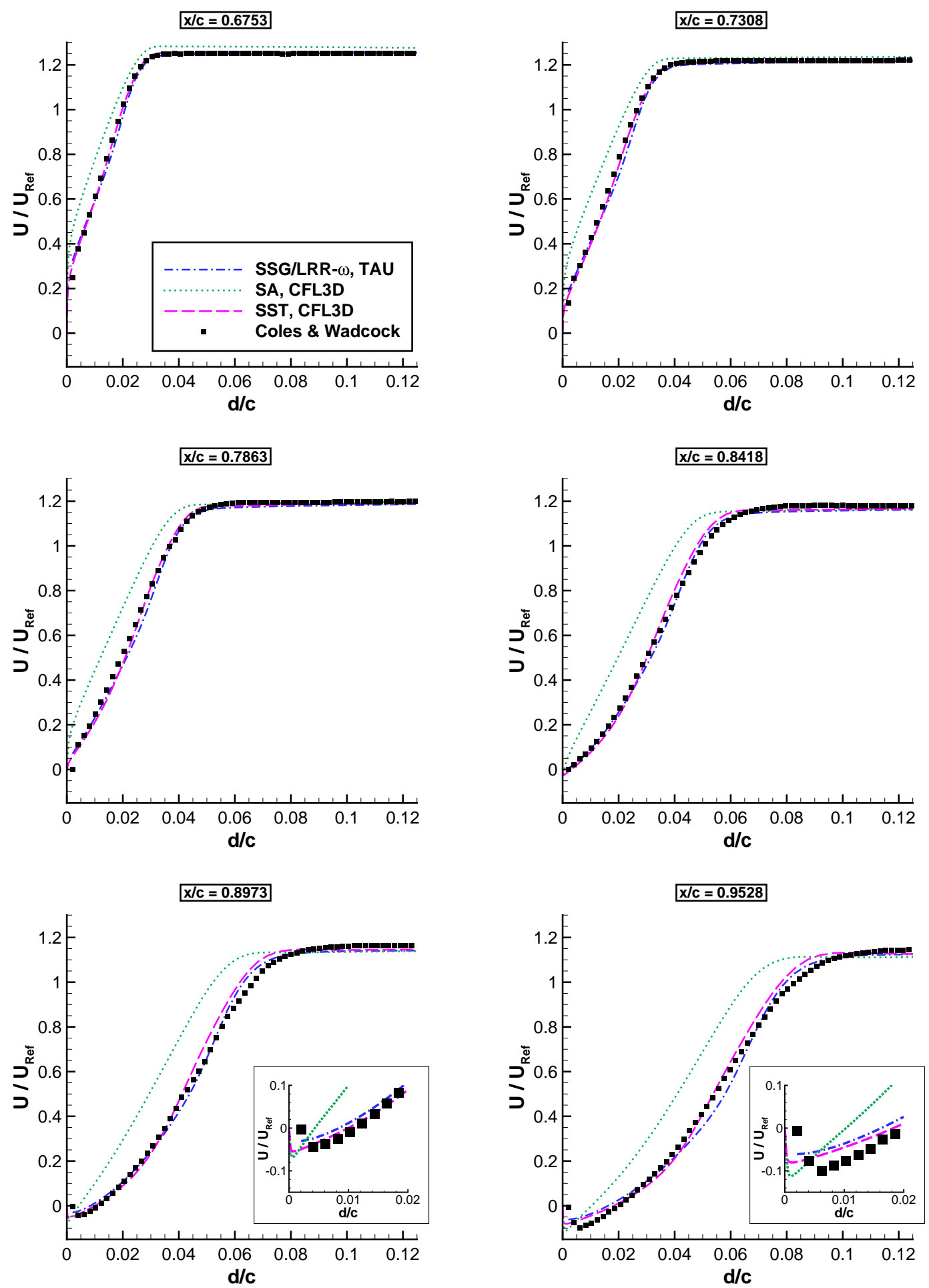

Figure 38. Subsonic flow around NACA 4412 airfoil. Non-dimensional streamwise velocity profiles at different streamwise positions. $d$ is the coordinate normal to the wall originating from the upper surface. Comparison of numerical results on the second finest mesh with $N=897 \times 257$ points with experiments by Coles and Wadcock. ${ }^{27,28}$ 

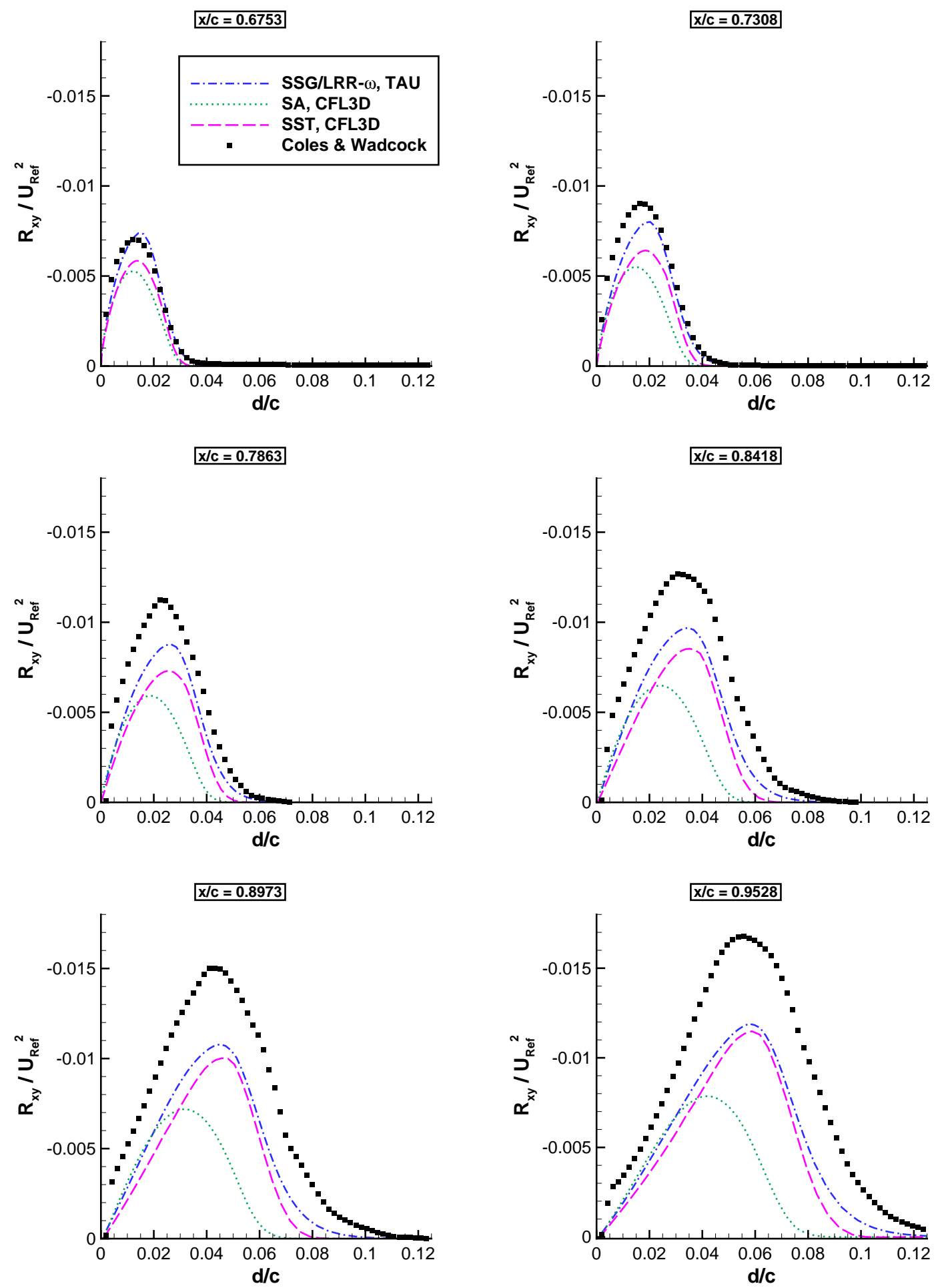

Figure 39. Subsonic flow around NACA 4412 airfoil. Profiles of non-dimensional Reynolds stress component $R_{x y}$ at different streamwise positions. $d$ is the coordinate normal to the wall originating from the upper surface. Comparison of numerical results on the second finest mesh with $N=897 \times 257$ points with experiments by Coles and Wadcock. ${ }^{27,28}$ 

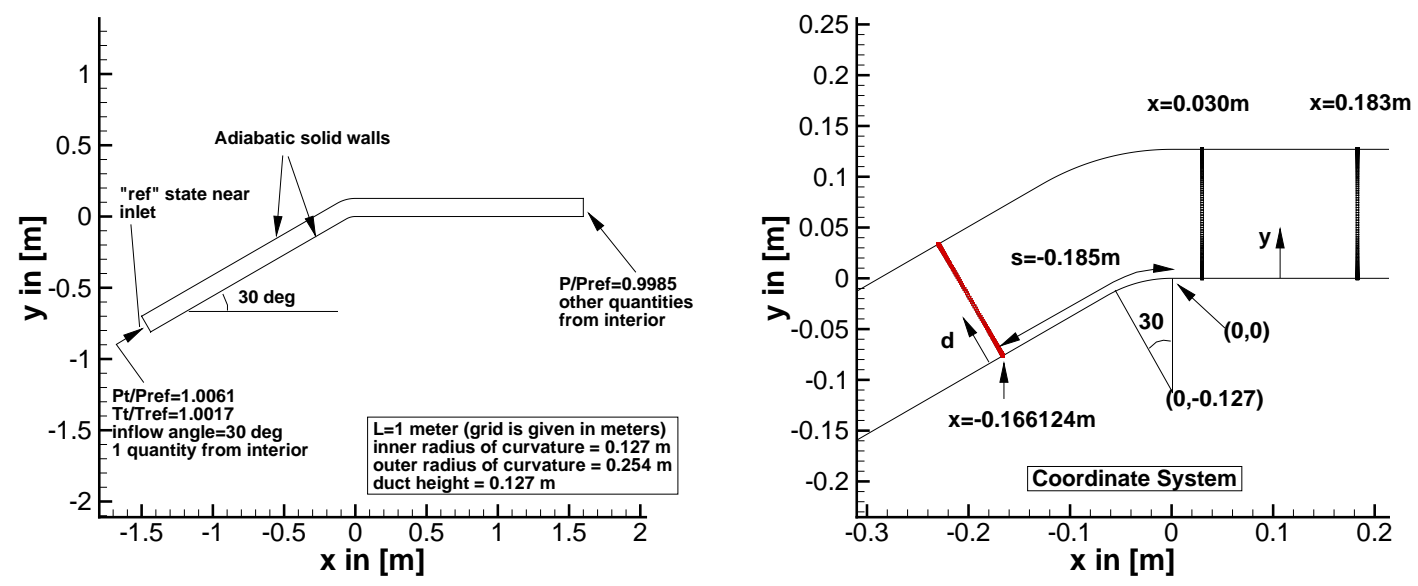

Figure 40. Flow through a curved duct. Boundary conditions and coordinate system, according to TMR Website. ${ }^{7}$

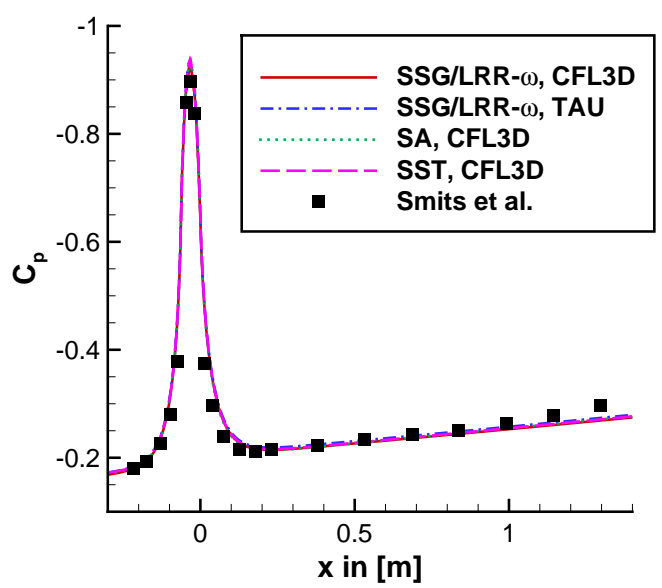

Figure 41. Flow through a curved duct. Pressure distribution in terms of pressure coefficient, $C_{p}$, on bottom wall. Comparison of numerical results on the second finest mesh with $N=513 \times 193$ points with experiments by Smits et al. ${ }^{29}$ According to TMR Website ${ }^{7}$ the numerical results are shifted by $\Delta C_{p}=-0.04$, in order to match the experimental reference.

\section{Bottom (convex) wall}

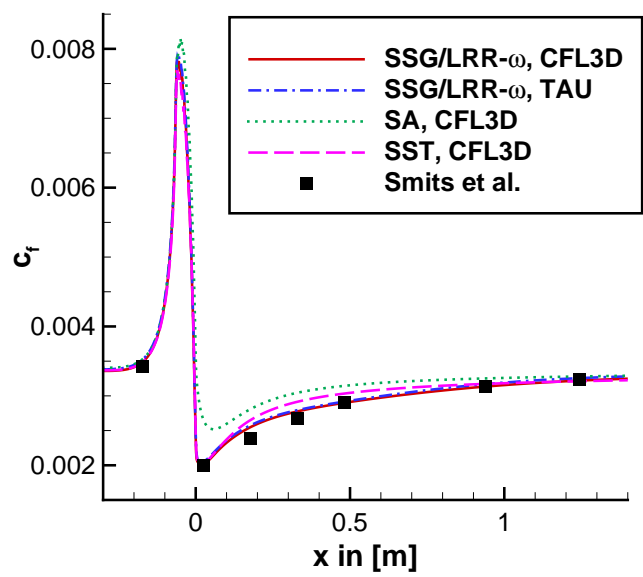

Top (concave) wall

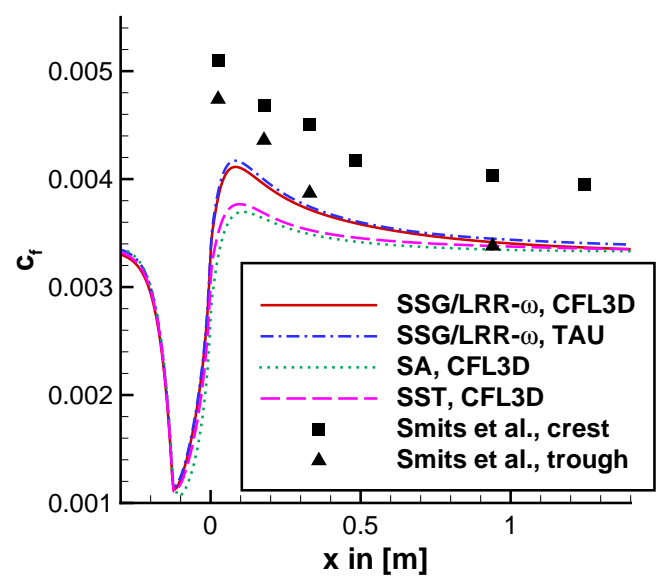

Figure 42. Flow through a curved duct. Skin friction distribution in terms of local skin friction coefficient on bottom wall (left) and top wall (right). Comparison of numerical results on the second finest mesh with $N=513 \times 193$ points with experiments by Smits et al. ${ }^{29}$ 

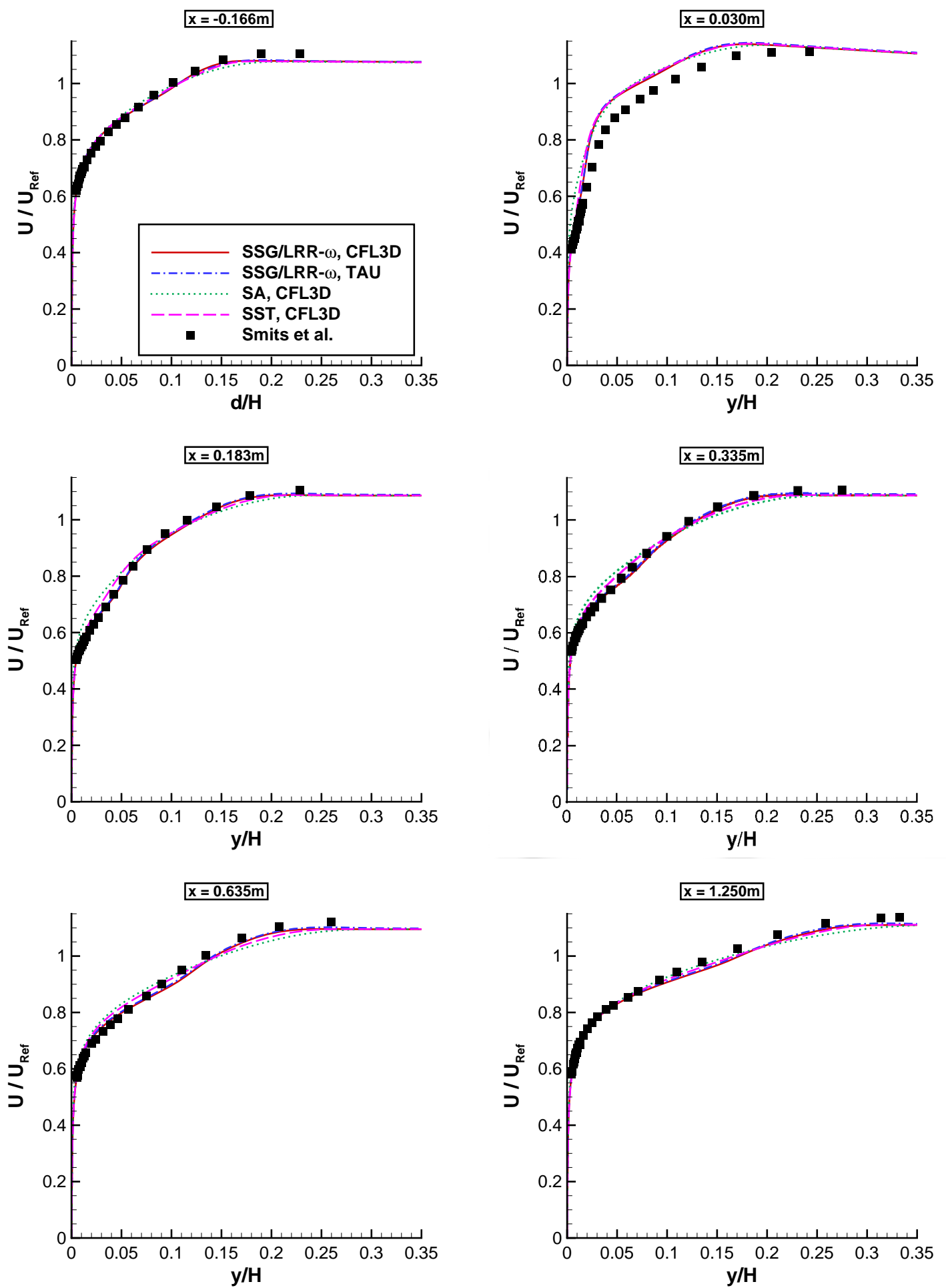

Figure 43. Flow through a curved duct. Non-dimensional velocity profiles at different streamwise positions. $d$ is the coordinate normal to the wall originating from the lower surface (see Fig. 40 (right)). Comparison of numerical results on the second finest mesh with $N=513 \times 193$ points with experiments by Smits et al. ${ }^{29}$ 

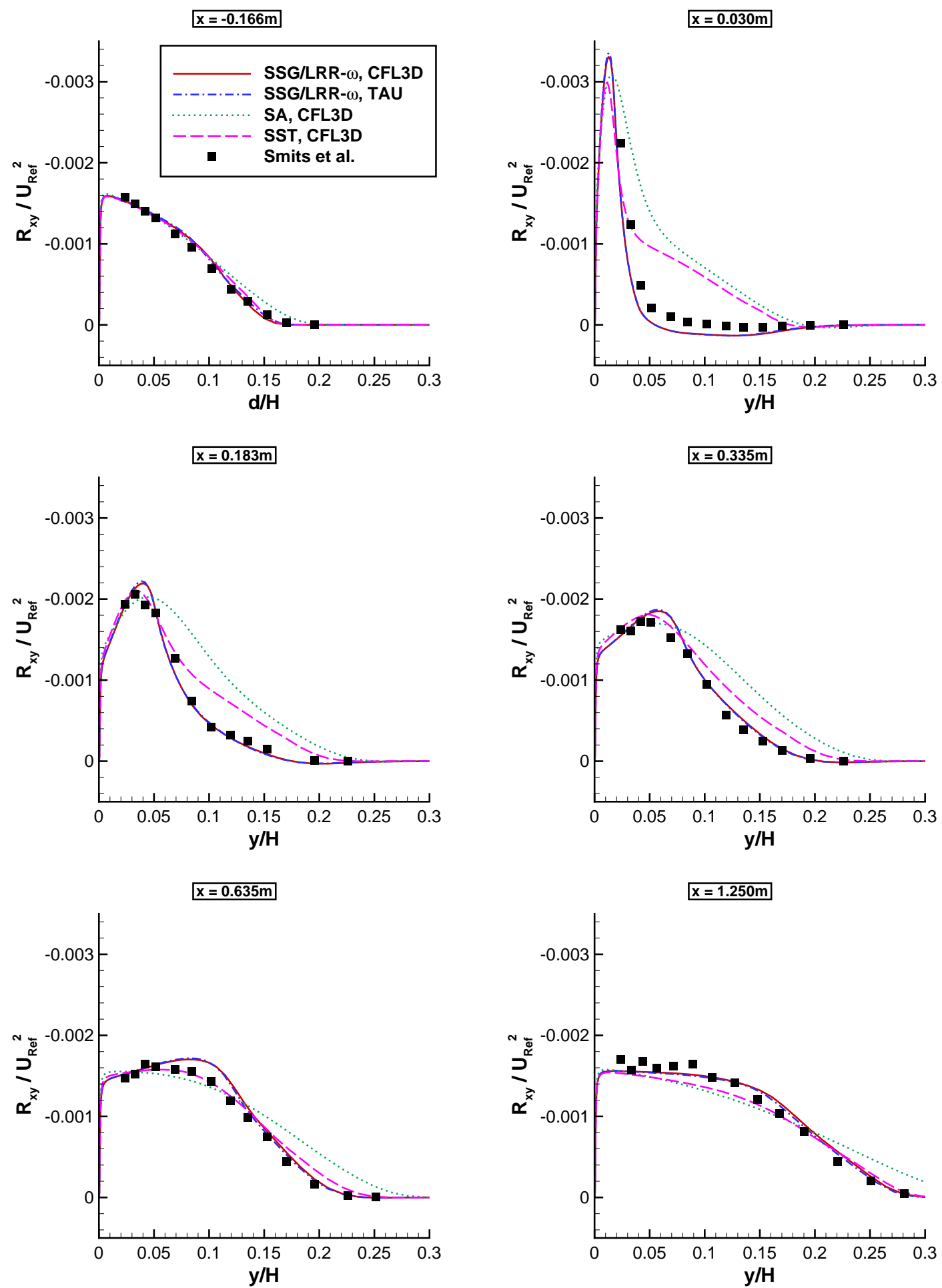

Figure 44. Flow through a curved duct. Non-dimensional Reynolds shear stress profiles at different streamwise positions. $d$ is the coordinate normal to the wall originating from the lower surface (see Fig. 40 (right)). Comparison of numerical results on the second finest mesh with $N=513 \times 193$ points with experiments by Smits et al. ${ }^{29}$ 

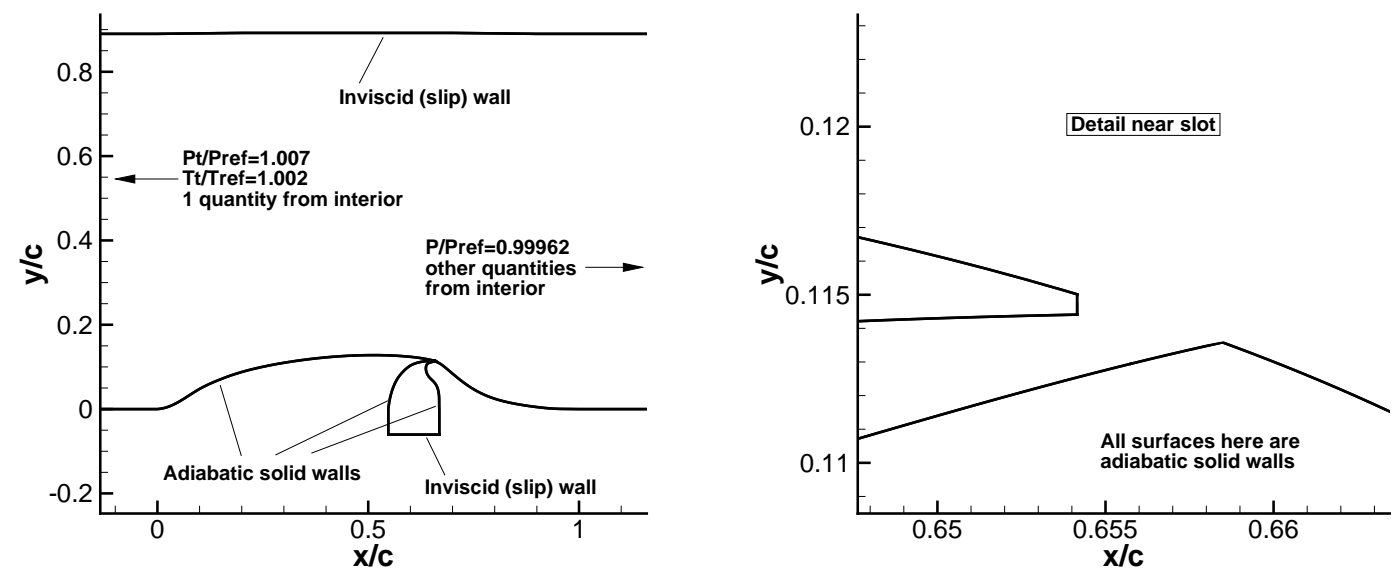

Figure 45. Two-dimensional flow over a wall-mounted hump. Boundary conditions according to TMR Website. ${ }^{7}$
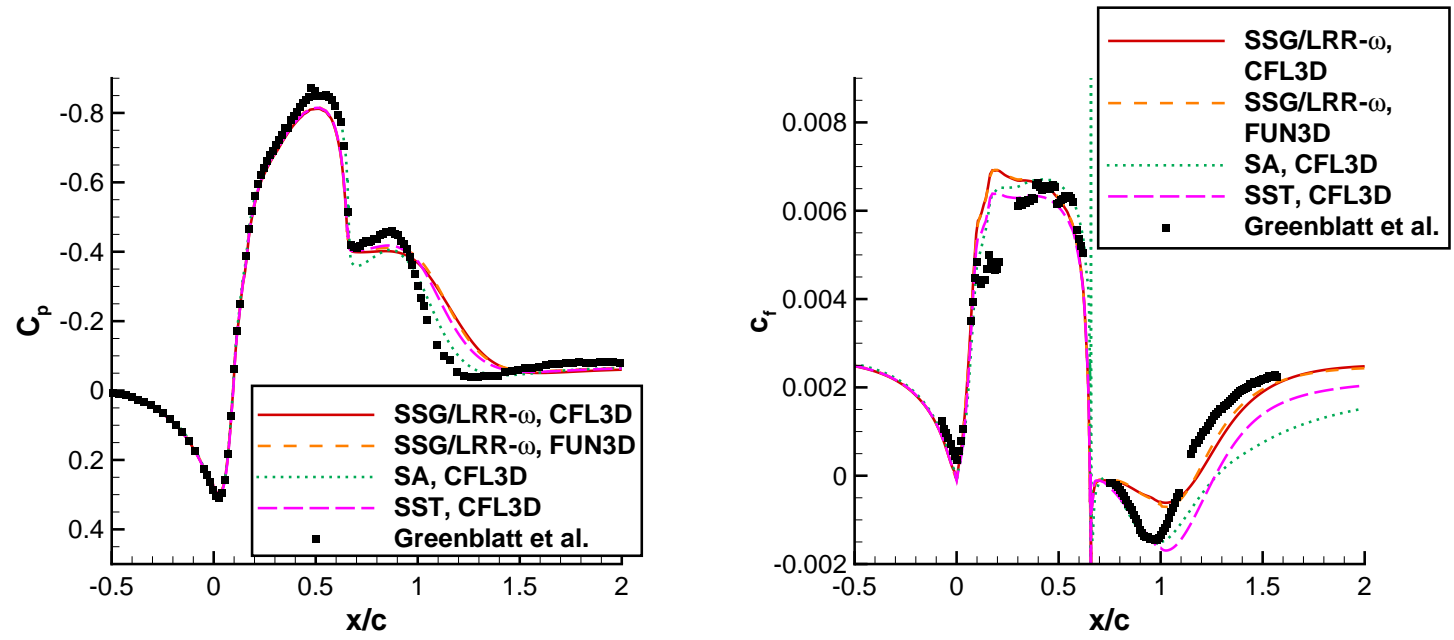

Figure 46. Two-dimensional flow over a wall-mounted hump. Pressure distribution (left) and skin friction distribution (right). Comparison of simulation results on the fine mesh with $N=210,060$ points with experiments by Greenblatt et al. ${ }^{30-32}$
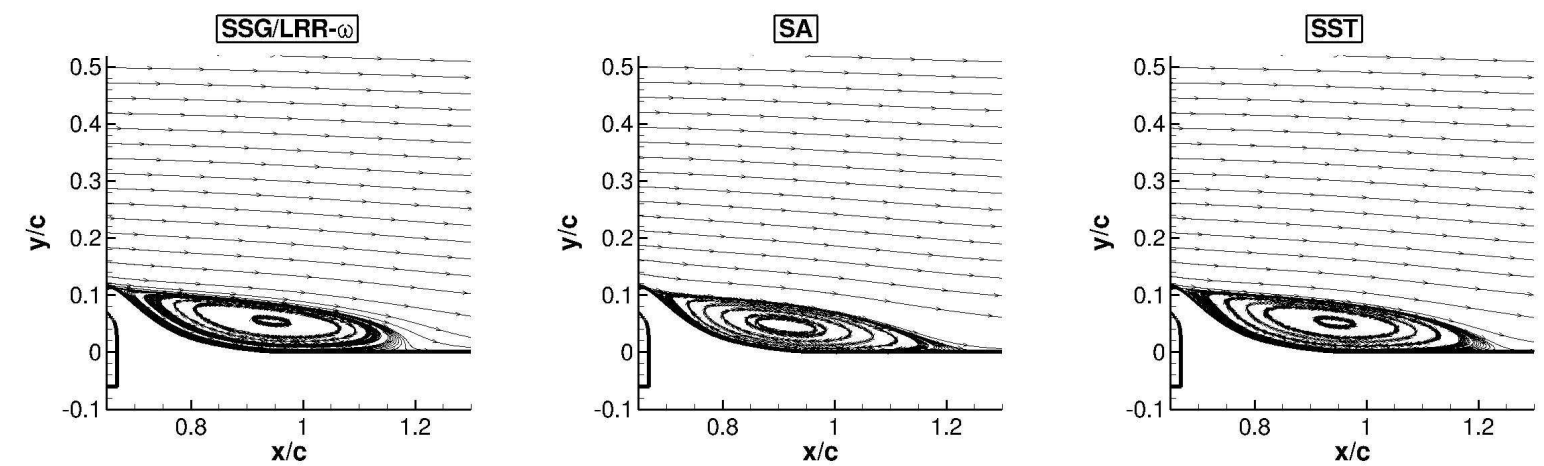

Figure 47. Two-dimensional flow over a wall-mounted hump. Streamlines in the region of flow separation predicted with CFL3D using the SSG/LRR- $\omega$ model (left), the SA model (center) and the SST model (right). 

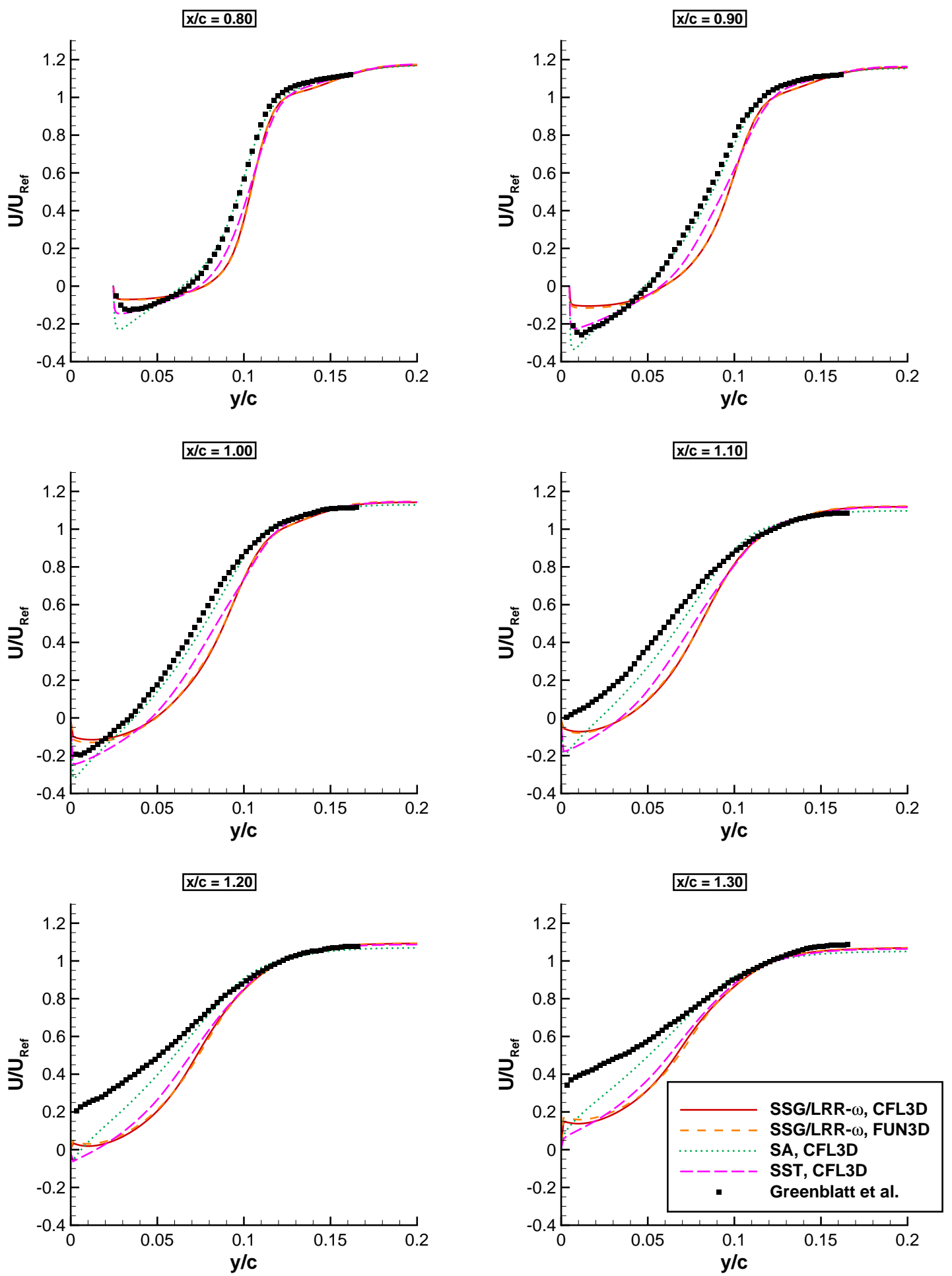

Figure 48. Two-dimensional flow over a wall-mounted hump. Profiles of the non-dimensional streamwise velocity component at different positions long the hump. Comparison of numerical results on the fine mesh with $N=210,060$ points with experiments by Greenblatt et al. ${ }^{30-32}$ 

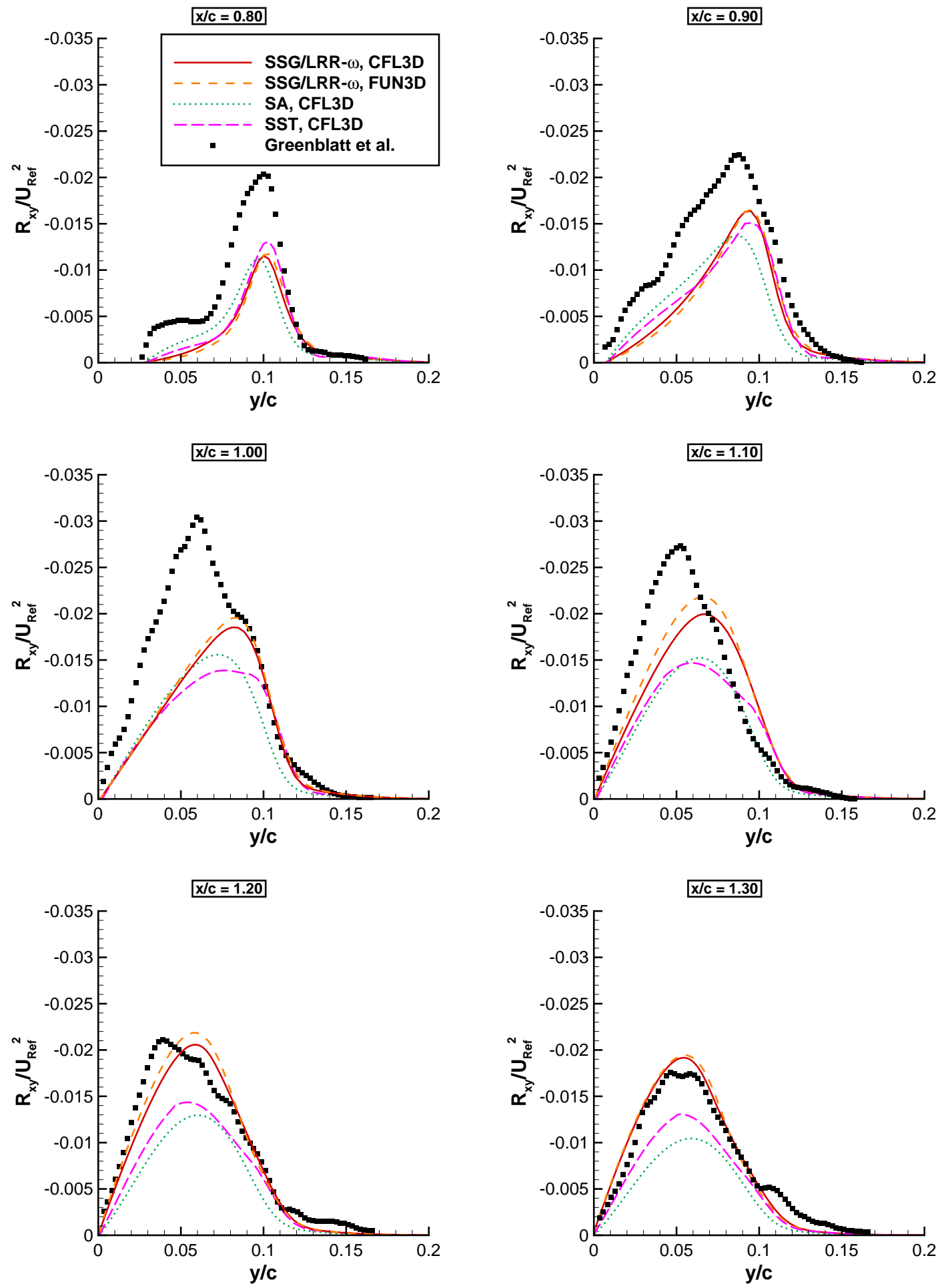

Figure 49. Two-dimensional flow over a wall-mounted hump. Profiles of the non-dimensional Reynolds stress component $R_{x y}$ at different positions long the hump. Comparison of numerical results on the fine mesh with $N=210,060$ points with experiments by Greenblatt et al. ${ }^{30-32}$ 

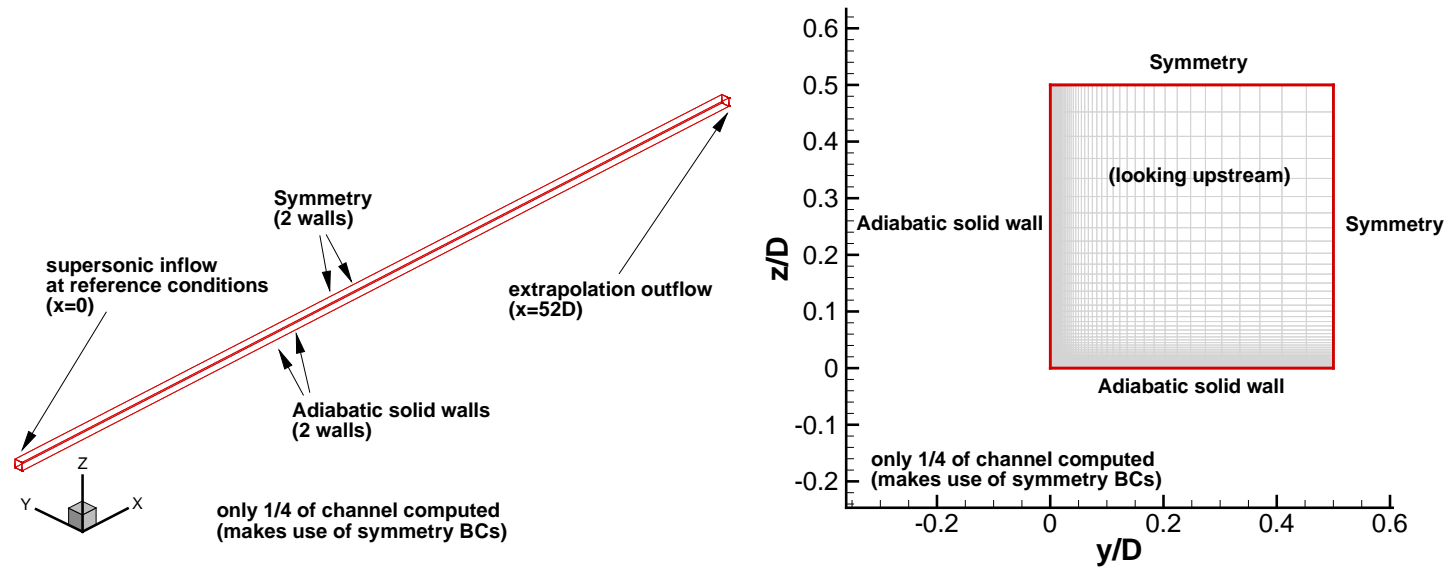

Figure 50. Supersonic flow through a square duct. Boundary conditions and grid structure, according to TMR Website. ${ }^{7}$

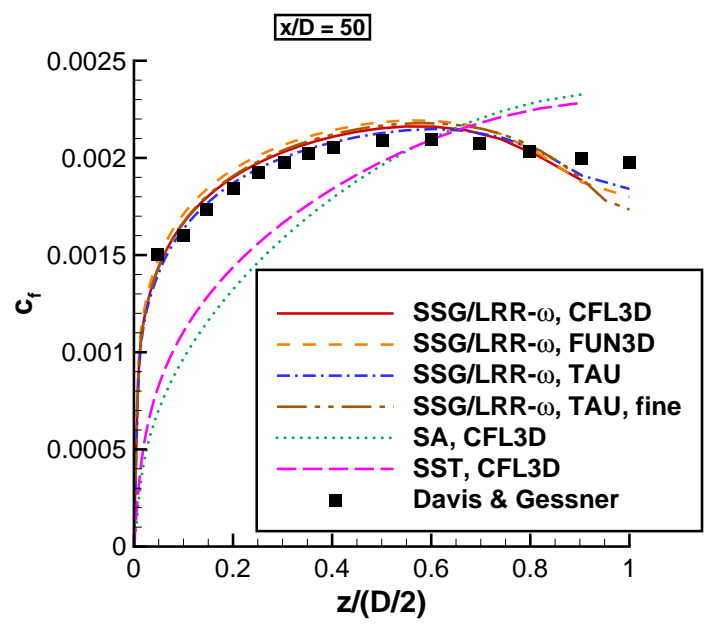

Figure 51. Supersonic flow through a square duct. Skin friction distribution on vertical wall. Comparison of numerical results on the second finest mesh with $N=481 \times 81 \times 81$ points with experiments by Davis and Gessner. ${ }^{34}$ "SSG/LRR- $\omega$, TAU, fine" refers to TAU result, using SSG/LRR- $\omega$ on the finest mesh. with $N=961 \times 161 \times 161$ points. 

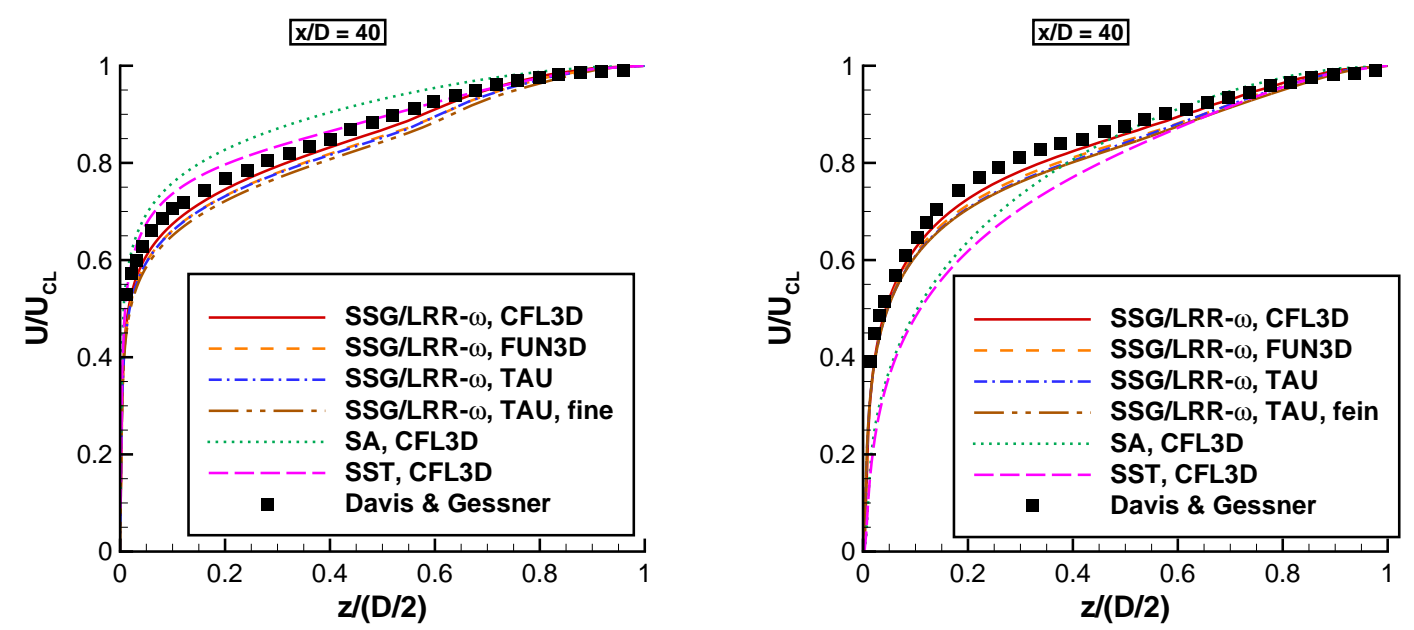

Figure 52. Supersonic flow through a square duct. Non-dimensional velocity at position $x / D=40$ along vertical centerline (left) and along the diagonal (right). Comparison of numerical results on the second finest mesh with $N=481 \times 81 \times 81$ points with experiments by Davis and Gessner. ${ }^{34}$ "SSG/LRR- $\omega$, TAU, fine" refers to TAU result, using SSG/LRR- $\omega$ on the finest mesh with $N=961 \times 161 \times 161$ points.
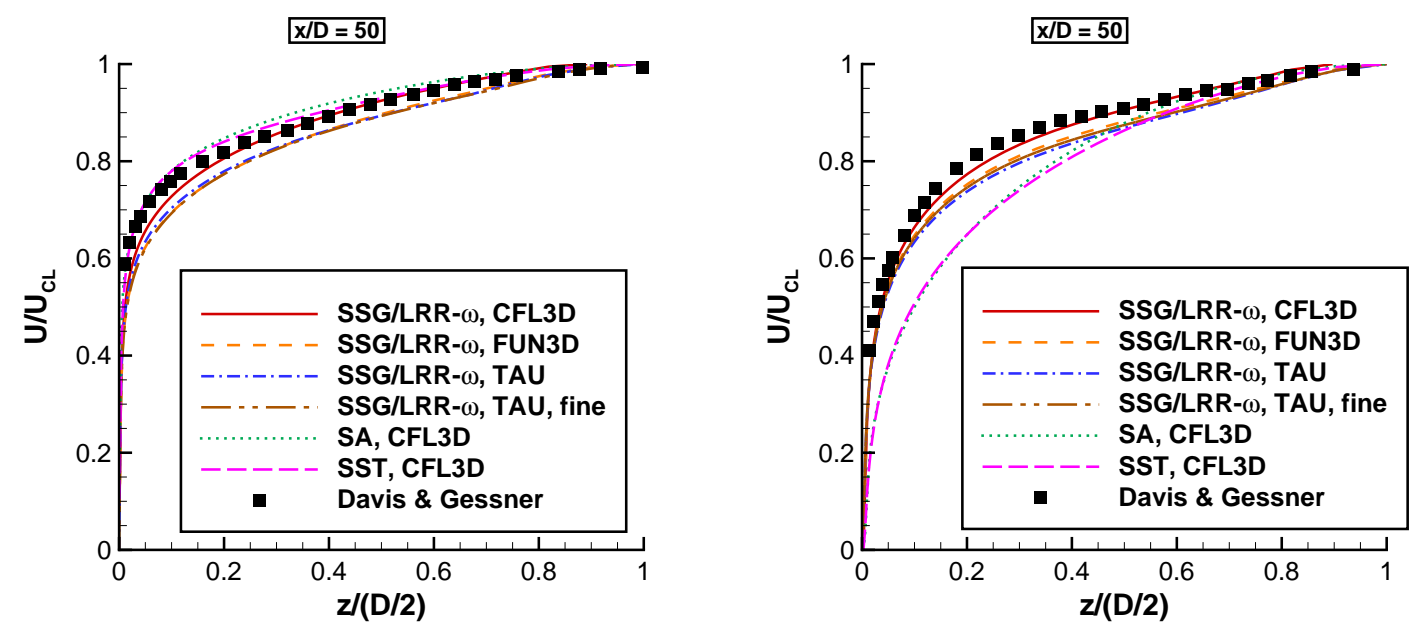

Figure 53. Supersonic flow through a square duct. Non-dimensional velocity at position $x / D=50$ along vertical centerline (left) and along the diagonal (right). Comparison of numerical results on the second finest mesh with $N=481 \times 81 \times 81$ points with experiments by Davis and Gessner. ${ }^{34}$ "SSG/LRR- $\omega$, TAU, fine" refers to TAU result, using SSG/LRR- $\omega$ on the finest mesh with $N=961 \times 161 \times 161$ points. 

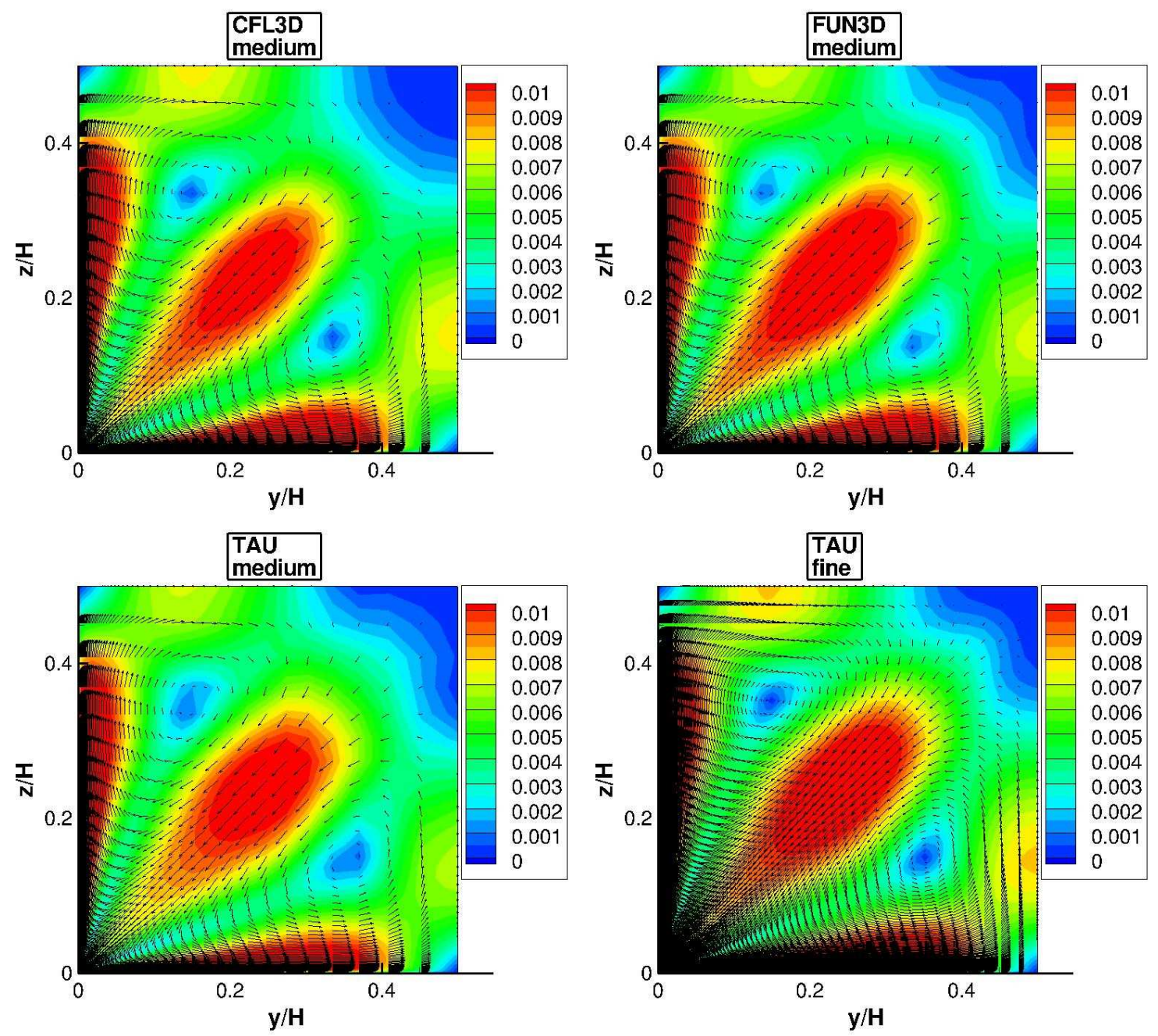

Figure 54. Supersonic flow through a square duct. Velocity field at $x / H=40$. Numerical results with CFL3D, FUN3D and TAU on the second finest mesh with $N=481 \times 81 \times 81$ points and with TAU on the finest mesh with $N=961 \times 161 \times 161$ points, using the SSG/LRR- $\omega$ model. Colors refer to absolute velocity in the plane, scaled with the streamwise centerline velocity. 